Check for updates

Cite this: RSC Adv., 2018, 8, 29714

\title{
Mechanistic evaluation of phytochemicals in breast cancer remedy: current understanding and future perspectives
}

\author{
Muhammad Younas, ${ }^{a}$ Christophe Hano, ${ }^{b}$ Nathalie Giglioli-Guivarc' $h^{c}$ \\ and Bilal Haider Abbasi (iD) *abc
}

Breast cancer is one of the most commonly diagnosed cancers around the globe and accounts for a large proportion of fatalities in women. Despite the advancement in therapeutic and diagnostic procedures, breast cancer still represents a major challenge. Current anti-breast cancer approaches include surgical removal, radiotherapy, hormonal therapy and the use of various chemotherapeutic drugs. However, drug resistance, associated serious adverse effects, metastasis and recurrence complications still need to be resolved which demand safe and alternative strategies. In this scenario, phytochemicals have recently gained huge attention due to their safety profile and cost-effectiveness. These phytochemicals modulate various genes, gene products and signalling pathways, thereby inhibiting breast cancer cell proliferation, invasion, angiogenesis and metastasis and inducing apoptosis. Moreover, they also target breast cancer stem cells and overcome drug resistance problems in breast carcinomas. Phytochemicals as adjuvants with chemotherapeutic drugs have greatly enhanced their therapeutic efficacy. This review focuses on the recently recognized molecular mechanisms underlying breast cancer chemoprevention with the use of phytochemicals such as curcumin, resveratrol, silibinin, genistein, epigallocatechin gallate, secoisolariciresinol, thymoquinone, kaempferol, quercetin, parthenolide, sulforaphane, ginsenosides, naringenin, isoliquiritigenin, luteolin, benzyl isothiocyanate, $\alpha$-mangostin, $3,3^{\prime}$-diindolylmethane, pterostilbene, vinca alkaloids and apigenin.

\author{
Received 7th June 2018 \\ Accepted 15th August 2018 \\ DOI: $10.1039 / \mathrm{c} 8 \mathrm{ra0} 4879 \mathrm{~g}$ \\ rsc.li/rsc-advances
}

\section{Introduction}

Breast cancer is one of the most commonly diagnosed cancers around the world and accounts for a large proportion of mortalities in females. The American Cancer Society estimated that in 2017, there will be an expected 63410 new cases of in situ, 252710 cases of invasive and 40610 cases of breast cancer mortalities across women in the United States alone. ${ }^{1,2}$ On the basis of expression of the estrogen receptor (ER), breast cancer is categorized into ER-positive and ER-negative types. The human epidermal growth factor receptor 2 (HER2) and progesterone receptor (PR) further divide breast cancer into several molecular subtypes like HER2-positive, luminal A and luminal B. These types of breast carcinomas respond to aromatase inhibitors or hormonal therapy. However, another type of breast cancer, known as basal-like or triple negative breast cancer (TNBC), lacks these three receptors and thus does not respond to hormonal

\footnotetext{
${ }^{a}$ Department of Biotechnology, Quaid-i-Azam University, Islamabad-45320, Pakistan. E-mail: bhabbasi@qau.edu.pk; Fax: +92-51-90644121; Tel: +92-51-90644121; +33767-97-0619

${ }^{b}$ Laboratoire de Biologie des Ligneux et des Grandes Cultures (LBLGC), Plant Lignans Team, UPRES EA 1207, Université d'Orléans, F 28000 Chartres, France

${ }^{c}$ EA2106 Biomolecules et Biotechnologies Vegetales, Universite Francois-Rabelais de Tours, Tours, France
}

therapy. ${ }^{3-8}$ Current therapeutic interventions in breast cancer remedy include radiation, surgical exclusion and the use of various chemotherapeutic drugs such as paclitaxel, doxorubicin, cisplatin, docetaxel, carboplatin, epirubicin, bevacizumab and cyclophosphamide. ${ }^{9,10}$ However, the incidences of drug resistance and serious side-effects associated with these treatment methods have greatly reduced their therapeutic potential. ${ }^{11}$ These complications propel researchers to look into alternative and safer chemotherapeutic strategies.

Human beings have always been suffered from infections by fungi, parasites, bacteria, viruses and many health disorders such as pain, inflammation, digestive complications and colds etc. ${ }^{12}$ Recent medicines, based on antibiotics and synthetic drugs, have come into practice during the previous 150 years. Before that, humans had to depend on drugs derived from plants, animals and fungi. The curing of health disorders and infections with herbal medicines includes active natural products, known as secondary metabolites or phytochemicals, which are found in almost all plant species. ${ }^{12}$ Secondary metabolites encompass a diverse group of organic compounds which have a vital role in plant defence systems and assist the interaction with the biotic environment. Many secondary metabolites like phenolics, alkaloids and terpenes are classified on the basis of their biosynthetic origin which possess different biological properties and are employed as pharmaceuticals, flavors, 
colors, agrochemicals, fragrances, food additives and biopesticides. ${ }^{13-15}$ Secondary metabolites specifically modulate a molecular target in humans or animals. Such targets often include neuroreceptors, ion pumps, ion channels and elements of the cytoskeleton or enzymes degrading neurotransmitters. ${ }^{12,16-20}$ An estimated more than 5000 distinct phytochemicals have been recognized in vegetables, fruits and grains, however, a large proportion is yet to be identified and understood before we can completely comprehend their health benefits in whole foods. ${ }^{21}$ Phytochemicals can be categorized into different classes such as phenolics, terpenes, organosulfur compounds and alkaloids (Fig. 1). ${ }^{393,394}$ The molecular structures of various phytochemicals involved in breast cancer chemoprevention with their major plant sources are given in Table 1.

Phytochemicals display diverse range of diseasepreventing or protective effects. They have been used since ancient times to cope with various ailments including cancer, diabetes, cardiovascular diseases, inflammation, neurological disorders and skin diseases etc. ${ }^{22-28}$ Numerous epidemiological studies have predicted reduced cancer incidence with the use of phytochemicals. ${ }^{29-31}$ Phytochemicals such as curcumin $^{32}$ resveratrol, ${ }^{33,34}$ epigallocatechin gallate (EGCG) ${ }^{35,36}$ silibinin, ${ }^{37}$ benzyl isothiocyanate, ${ }^{38,39}$ genistein, ${ }^{40}$ kaempferol, ${ }^{\mathbf{4 1 , 4 2}}$ thymoquinone, ${ }^{\mathbf{4 3}}$ quercetin, ${ }^{\mathbf{4 4}}$ parthenolide, ${ }^{\mathbf{4 5}}$ sulforaphane, ${ }^{46}$ naringenin, ${ }^{47,327,330}$ isoliquiritigenin ${ }^{48,49}$ and ginsenosides ${ }^{50-52}$ have been shown to suppress breast carcinoma via modulation of various signalling transduction pathways, genes and gene products. These phytochemicals exert anti-breast cancer effects by inducing cellular apoptosis and reducing cell proliferation through modulation of various targets (Fig. 2 \& 4). Additionally, phytochemicals inhibit the angiogenesis, metastasis and migratory behaviours in breast cancer cells (Fig. 3 \& 4). Moreover, these compounds greatly enhanced the therapeutic efficacy of different anti-cancer drugs, overcame drugs resistance in breast cancer cells and also achieved sensitization to radiations. ${ }^{53-58}$ The compounds targeted breast cancer stem cells (bCSCs)/progenitor cells. ${ }^{\mathbf{5 9 , 6 0}}$ CSCs are mainly involved in promotion of invasion, metastasis, abnormal proliferation, recurrence and drug resistance. ${ }^{61}$ The self-renewal capability of CSCs is related to the regulatory pathways of Notch, Wnt/ $\beta$ catenin, hedgehog and P13K/Akt. These pathways might also contribute in the maintenance of $\mathrm{CD} 44^{+} / \mathrm{CD} 24^{-/ \text {low }}$ bCSCs stemness. ${ }^{59,62}$ Dandawate et al. (2016) reviewed the role of phytochemicals in targeting bCSCs. ${ }^{60}$ Abdal Dayem et al. (2016) reviewed the effect of polyphenols against breast cancer and CSCs (Fig. 5). ${ }^{395}$ Petric et al. (2015) reviewed some phytochemicals modulating signalling pathways in breast and hormone related cancers. ${ }^{63}$ Moreover, Siddiqui et al. (2015) have also put some light on therapeutic effect of phytochemicals in breast cancer. ${ }^{64}$ In this review article, we summarize various phytochemicals that are involved in breast cancer prevention, by putting in view the recently recognized molecular mechanisms, which may serve useful in future drugs development.

\section{Literature search}

This review was conducted using various search engines including Google Scholar, PubMed, and Scopus by putting keywords such as breast cancer, chemoprevention, phytochemicals and individual names of respective phytochemicals plus breast cancer. All the research articles included in here are from the last half decade: 2012-2018.

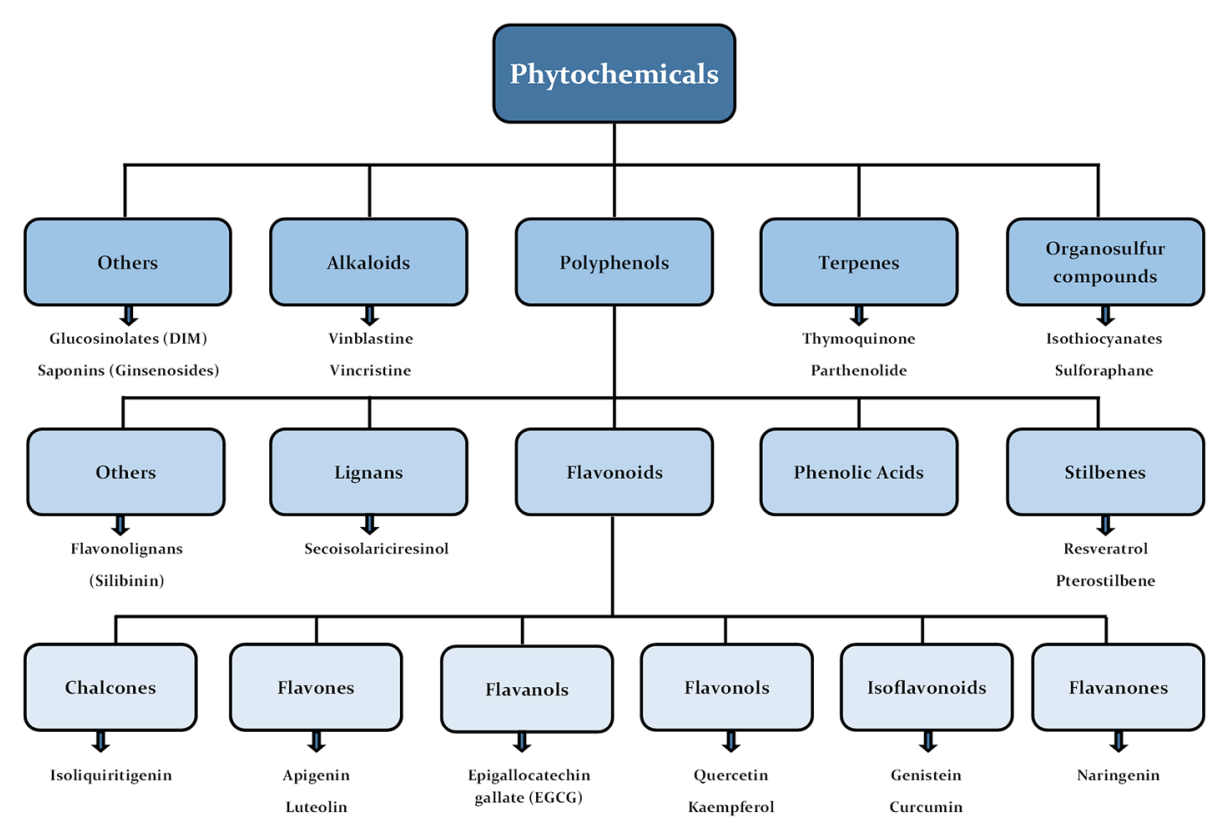

Fig. 1 Classification of phytochemicals involved in breast cancer chemoprevention. 


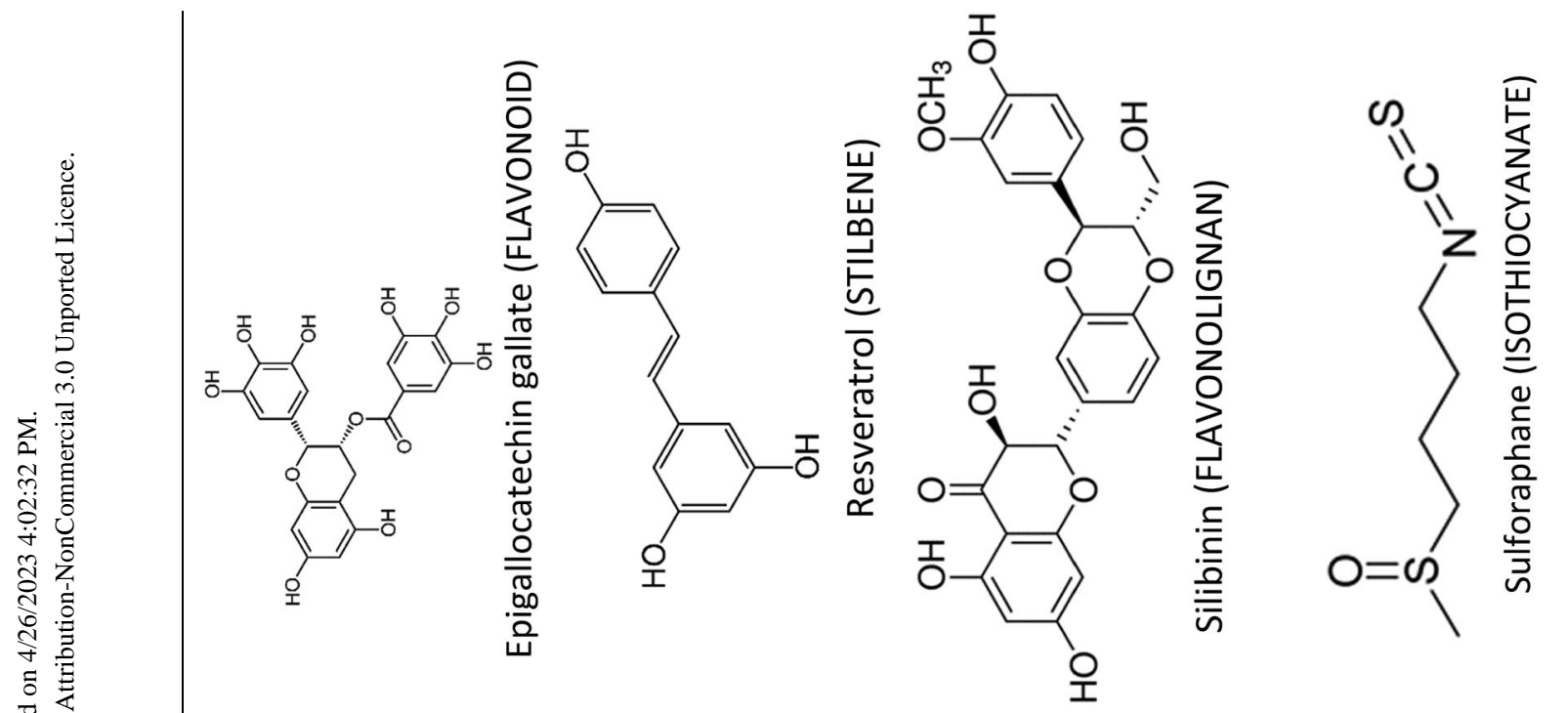

.
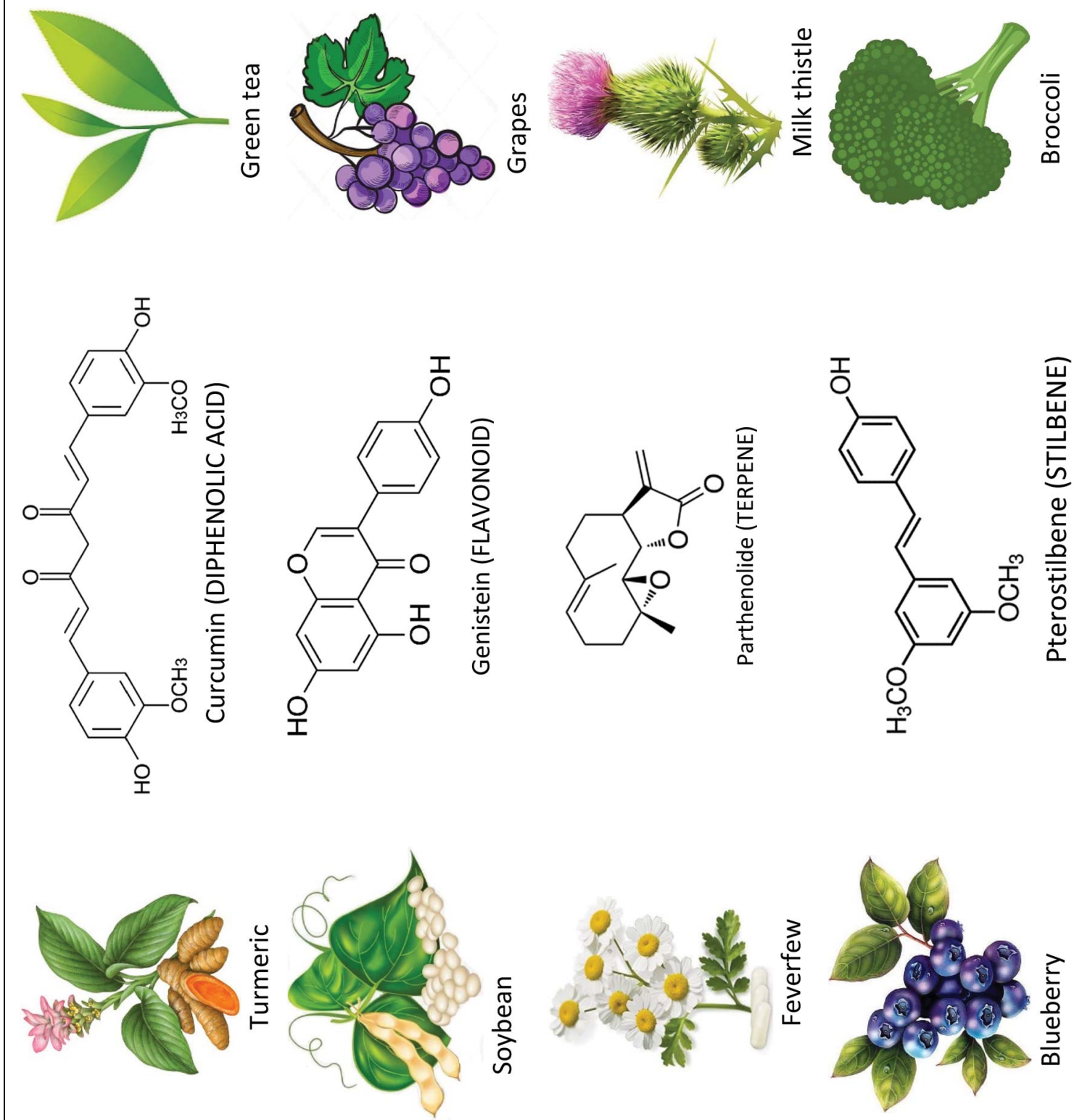

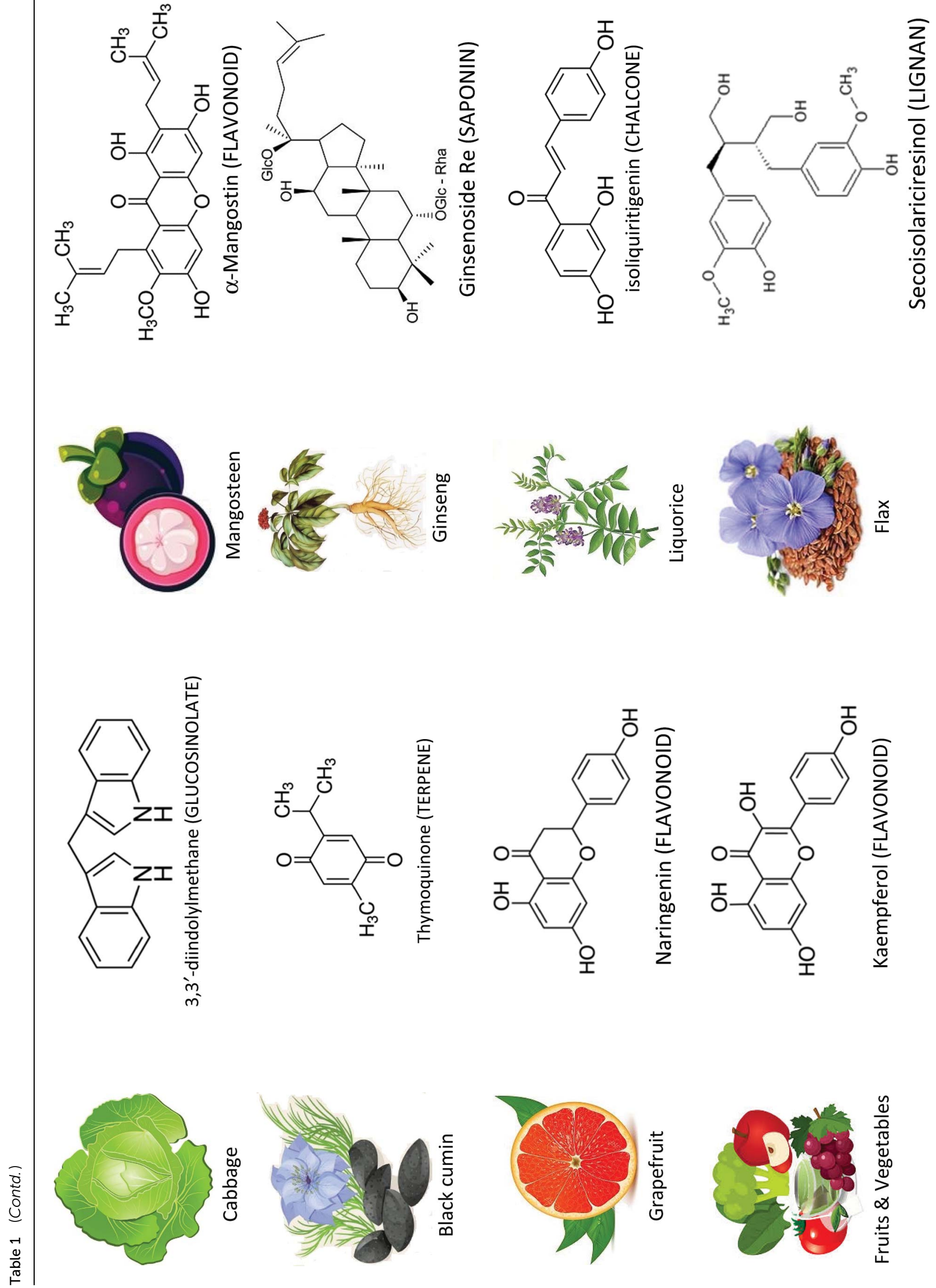


\section{(4) 3 Phytochemicals involved in breast cancer chemoprevention}

\subsection{Curcumin}

Curcumin - a yellow pigment found in the rhizome of turmeric-is one of the most extensively studied phytochemical in breast cancer therapy. It displayed a wide spectrum of activities such as anti-proliferative, apoptotic, anti-metastatic, anti-angiogenic, radio-protective, chemo-sensitization, induction of cell cycle arrest and inhibition of migration and invasion in breast cancer cells (Table 2). Curcumin can modulate the activities and functions of various gene products like enzymes, inflammatory cytokines, transcription factors and products related to cell proliferation and cell survival. ${ }^{65}$ Liu and Chen (2013) reviewed the effect of curcumin on breast cancer cells and showed that the compound modulate various molecular targets (Fig. 6). ${ }^{396}$

Recently, curcumin was shown to induce apoptosis in MCF-7 cells through increased caspase- 3 and caspase-9 expressions. Additionally, this compound suppressed miR-21 expression via up-regulation of PTEN/Akt signalling. ${ }^{66}$ Curcumin also reduced the proliferation of MCF-7 cells by decreasing nitric oxide and ROS levels. Synergistic anti-proliferative effect was observed when ECM proteins: fibronectin or collagen were used along with curcumin. ${ }^{67}$ Moreover, the compound upregulated Bax and downregulated Bcl-2 expressions in MDA-MB-231 cells, leading to growth inhibition and apoptosis induction. ${ }^{68}$ Fatty acid synthase inhibition by curcumin also resulted in breast cancer cells apoptosis. ${ }^{69}$ Curcumin downregulated flap endonuclease 1 (DNA repair enzyme) expression via Nrf2 stimulation thereby reduced the proliferation of MCF-7 cells. ${ }^{70}$

Similarly, curcumin inhibited the nuclear translocation of $\beta$ catenin, hence impeded the trans-activation of slug and subsequent restoration of E-cadherin which led to increased formation of E-cadherin/ $\beta$-catenin complex and more $\beta$-catenin in cytosol to finally suppress bCSCs migration and EMT. ${ }^{71}$ Recently, hypomethylation reactivation of tumor suppressors by curcumin has also been presented. The compound reversed the hypermethylation status of Glutathione $S$-Transferase Pi 1 (GSTP1) gene in MCF-7 cells, leading to its reactivation. ${ }^{72}$ Likewise, it also activated Deleted in Liver Cancer 1 (DLC1) promoter by decreasing its methylation level via downregulation of $\mathrm{Sp} 1$ transcription factor to constrain the expression of DNA methyltransferase 1 (DNMT1) in MDA-MB-361 cells. ${ }^{73}$ Curcumin was also shown to decrease the methylation status of tumor suppressor termed as Ras-association domain family protein 1A (RASSF1A), thereby activating it. This was achieved through interference of curcumin with DNMT1. ${ }^{74}$

Co-treatment of curcumin with other drugs/phytochemicals have greatly enhanced their therapeutic efficacy in various malignancies, including breast cancer. Recently, the combined effect of curcumin and metformin (oral anti-diabetic drug) was explored in breast cancer cell lines which markedly suppressed VEGF expression, activated Th2 immune response and induced Trp53-independent apoptosis. ${ }^{75}$ Similarly, arabinogalactana polysaccharide obtained from larch wood-in combination
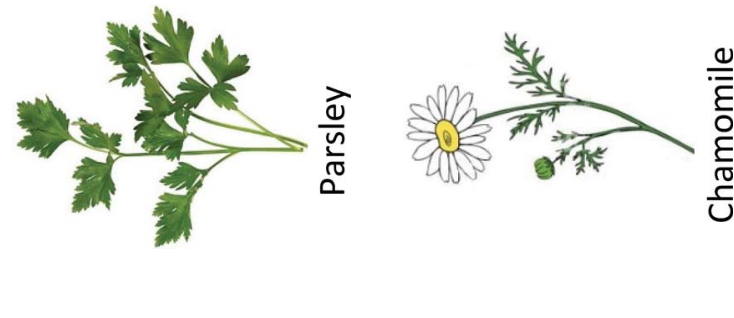


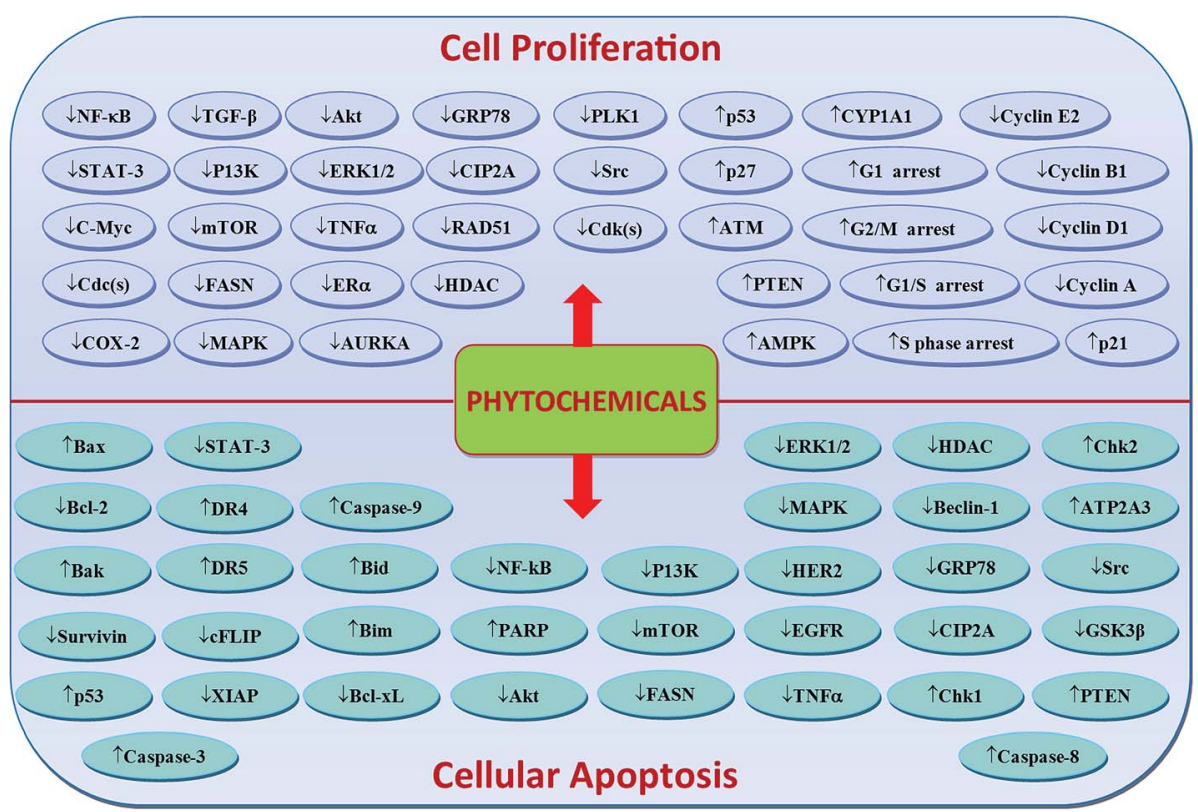

Fig. 2 Phytochemicals-mediated regulation of molecular targets/signalling pathways involved in breast cancer cells proliferation and apoptosis. Downward arrows $(\downarrow)$ represent downregulation while upward arrows $(\uparrow)$ represent upregulation.

with curcumin induced in vivo and in vitro apoptosis in breast cancer cells by increasing reactive oxygen species (ROS), cleaved-caspase-3 and Bax/Bcl-2 ratio. Reduced glutathione, p53 overexpression and a change in mitochondrial membrane were also observed..$^{76,77}$ Curcumin synergistically enhanced the effects of retinoic acid (RA) and IFN- $\beta$ on breast cancer cells by upregulating GRIM-19 (cell death regulatory gene) via STAT3dependent and- independent pathways. ${ }^{78}$ RA induction of apoptosis via CRABPII/RAR signalling pathway has been reported and curcumin was recently shown to upregulate CRABPII and RAR thus overcoming RA-resistance in TNBC cell lines. ${ }^{79}$ Similarly, curcumin sensitized MDR breast cancer cells to cisplatin via downregulation of CCAT1 and inactivation of P13K/Akt/mTOR pathway. ${ }^{80}$ Moreover, curcumin chemosensitized breast cancer cells to 5-fluorocuracil (drug which

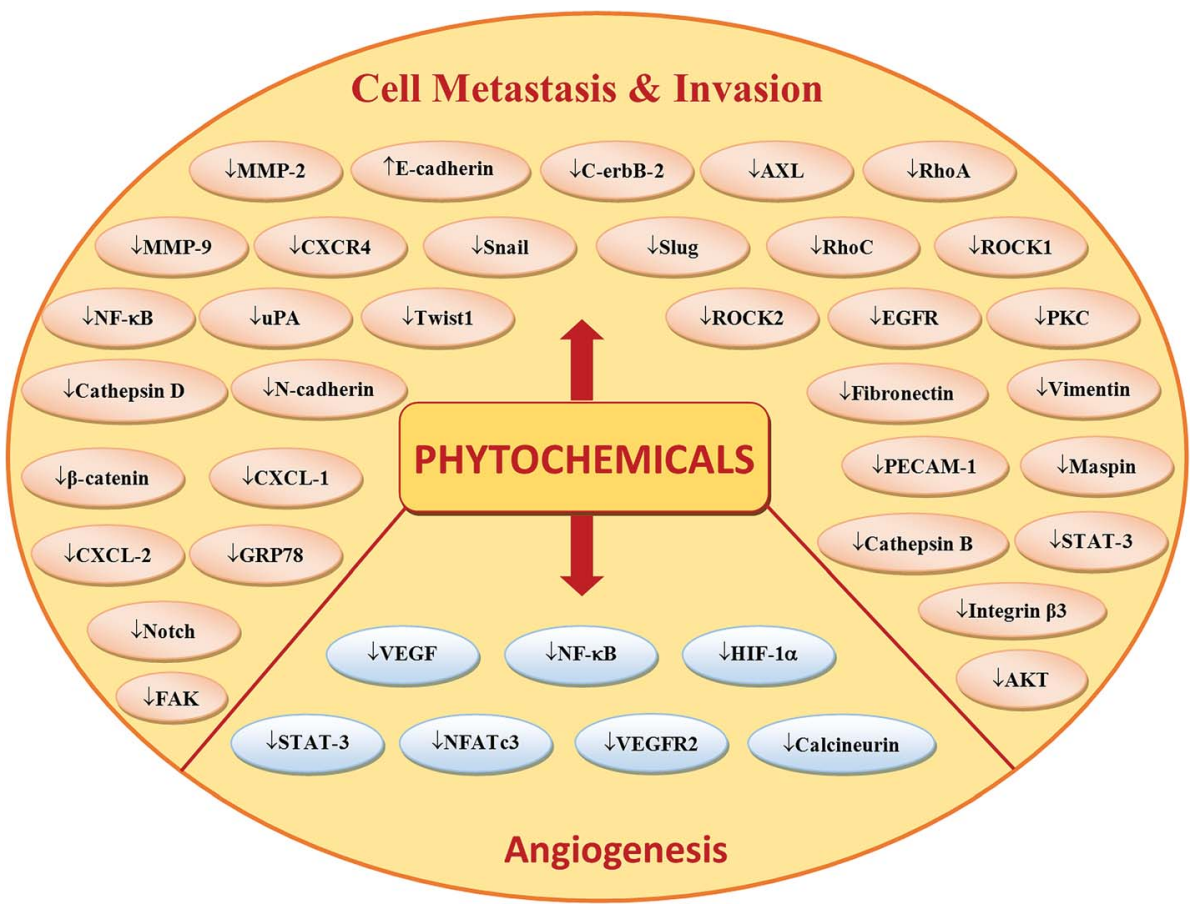

Fig. 3 Phytochemicals-mediated regulation of molecular targets/signalling pathways involved in breast cancer cells invasion, metastasis and angiogenesis. Downward arrows $(\downarrow)$ represent downregulation while upward arrows $(\uparrow)$ represent upregulation. 


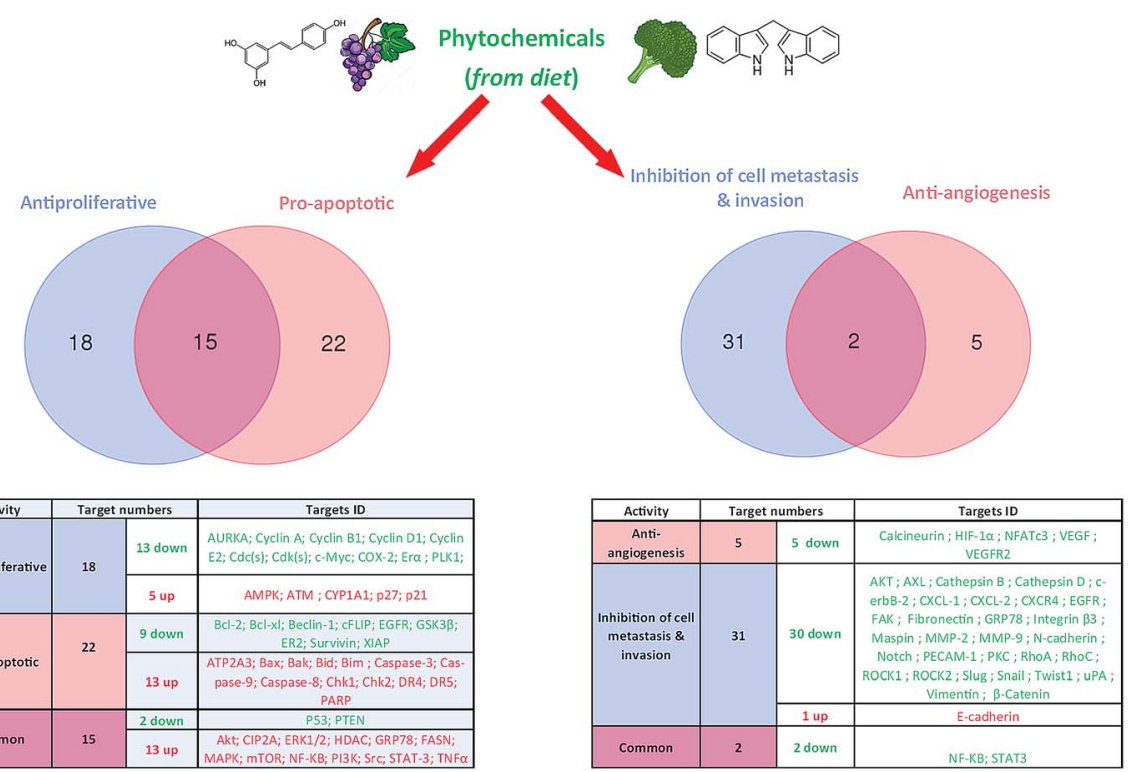

Fig. 4 Possible number of key targets involved in breast cancer cells proliferation, apoptosis, angiogenesis, invasion and metastasis and their regulation by phytochemicals.

exerts its action by inhibiting thymidylate synthase (TS)) through TS-dependent downregulation of NF- $\kappa \mathrm{B} .^{81}$

Curcumin inhibited the invasion and adhesion of MCF-7 cells by reducing the expression of UPA via activation of NF$\kappa \mathrm{B} .{ }^{82}$ Furthermore, the compound suppressed lysophosphatidic acid-induced invasion and metastasis in MCF-7 cells by interfering with RhoA/ROCK/MMPs pathway. ${ }^{83}$ Similarly, inhibition of angiogenesis and tumor growth through downregulation of $\mathrm{NF}-\kappa \mathrm{B}$ and its related gene products like PECAM-1, p65 and cyclin D1 by curcumin in MDA-MB-231 cells has also been reported. ${ }^{84}$ Curcumin upregulated $\mathrm{p} 16^{\mathrm{INK} 4 \mathrm{~A}}$ and several other tumor suppressors, inhibited JAK2 and STAT3 pathways, leading to decreased $\alpha$-smooth muscle actin and invasion/ migration abilities of breast cancer-associated fibroblasts. Curcumin also suppressed lamin B1 and triggered p16 ${ }^{\mathrm{INK} 4 \mathrm{~A}}$ dependent senescence in these fibroblasts. ${ }^{85}$ Matrix metalloproteinases (MMPs) possess a significant part in remodelling of the extracellular matrix. Tissue inhibitor of metalloproteinases (TIMPs) regulate the activities of MMPs. Curcumin regulated cell metastasis by up-regulating expression of

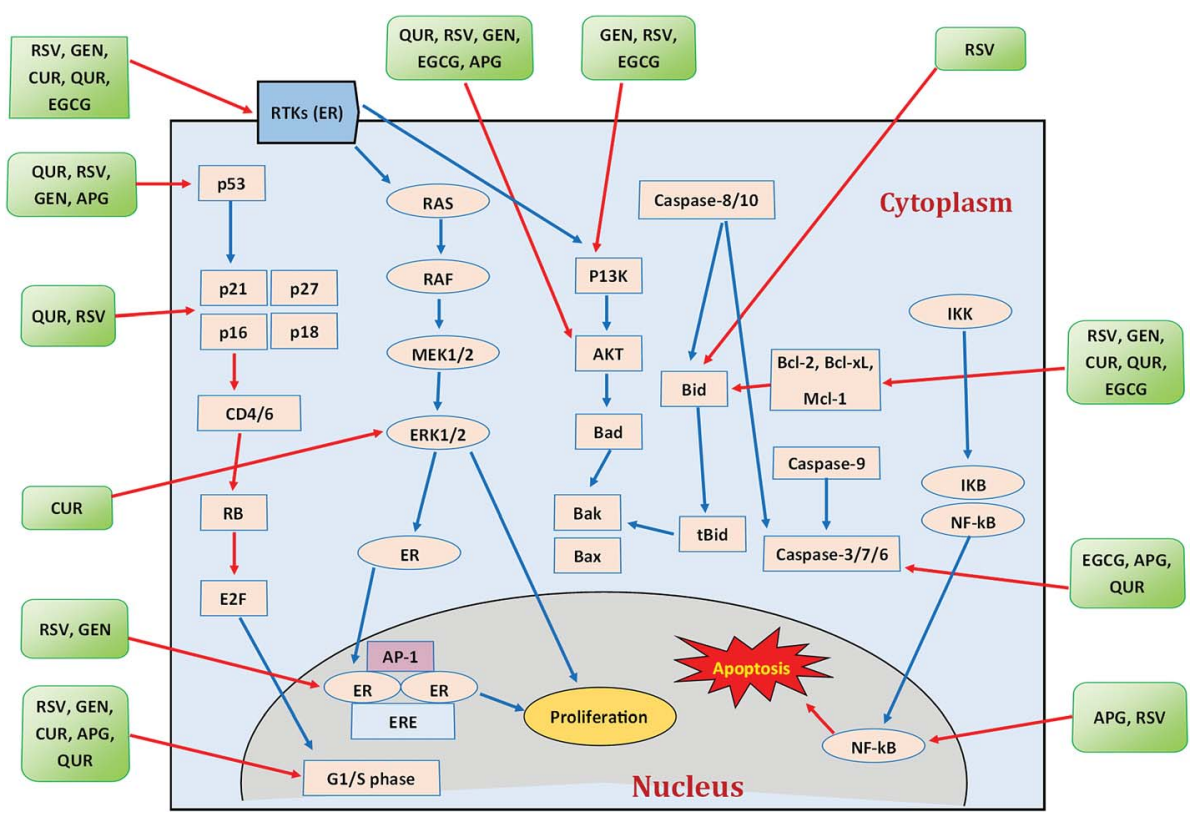

Fig. 5 Polyphenols and their modes of action against breast cancer. Blue arrows indicate activation while red arrows indicate inhibition. CUR $=$ curcumin, RSV = resveratrol, GEN = genistein, APG = apigenin, QUR = quercetin. Idea adopted from Abdal Dayem et al. (2016).395 
Table 2 Phytochemicals involved in breast cancer chemoprevention, their proposed mechanisms and effects

\begin{tabular}{|c|c|c|c|c|}
\hline Phytochemical & Cell line/model & Proposed mechanism & Effect & Ref. \\
\hline \multirow[t]{23}{*}{ Curcumin } & MCF-7 & $\downarrow$ GSTP1 methylation & $\uparrow$ Glutathione $S$-transferase Pi 1 & 72 \\
\hline & MDA-MB-361 & $\downarrow$ Sp1 expression, $\downarrow$ DLC1 methylation & $\begin{array}{l}\downarrow \text { Growth, } \uparrow \text { re-expression } \\
\text { of the tumor suppressor: } \\
\text { deleted in liver cancer } 1\end{array}$ & 73 \\
\hline & MCF-7 & $\begin{array}{l}\downarrow \text { miR-21, } \uparrow \text { PTEN, } \uparrow \text { Akt, } \uparrow \text { caspase- } 3, \\
\uparrow \text { caspase-9 }\end{array}$ & $\uparrow$ Apoptosis & 66 \\
\hline & MCF-7 \& MCF-7/DPP & $\begin{array}{l}\downarrow \text { CCAT1 expression, } \downarrow \text { PI3K, } \downarrow \text { p-Akt, } \\
\downarrow \text { p-mTOR }\end{array}$ & $\uparrow$ Autophagy, $\uparrow$ sensitization to cisplatin & 80 \\
\hline & $\begin{array}{l}\text { MCF-10F, MDA-MB-231 \& } \\
\text { Tumor } 2\end{array}$ & $\begin{array}{l}\downarrow \beta \text {-Catenin, } \downarrow \text { N-cadherin, } \downarrow \text { E-cadherin, } \\
\downarrow \text { slug, } \downarrow \text { Twist } 1, \downarrow \text { AXL }, \downarrow \text { fibronectin, } \downarrow \text { vimentin }\end{array}$ & $\downarrow$ Migration, $\downarrow$ invasion & 88 \\
\hline & MDA-MB-231 & $\uparrow \mathrm{AMPK}, \downarrow$ Akt & $\uparrow$ Autophagy, $\downarrow$ proliferation, $\downarrow$ migration & 280 \\
\hline & MDA-MB-231 & $\downarrow$ Intracellular FAS & $\uparrow$ Apoptosis & 69 \\
\hline & MDA-MB-231 & $\downarrow$ Slug/HK2 axis & $\uparrow$ Apoptosis, $\downarrow$ 4-OHT resistance & 281 \\
\hline & MCF-7, MCF10A, SUM149 & $\begin{array}{l}\downarrow \text { SCD }, \downarrow \text { CD49f, } \downarrow \text { LDH1A3 }, \downarrow \text { TP63, } \\
\downarrow \text { PROM1 }\end{array}$ & $\downarrow$ bCSCs self-renewal & 282 \\
\hline & MCF-7 & $\downarrow$ Cyclin B1, $\downarrow$ Cdc2 & $\downarrow$ Colonization & 283 \\
\hline & MCF-7 & $\begin{array}{l}\downarrow \text { RhoA, } \downarrow \text { ROCK1, } \downarrow \text { ROCK2, } \downarrow \text { MMP- } 2, \\
\downarrow \text { MMP-9 }\end{array}$ & $\downarrow$ Invasion & 83 \\
\hline & MDA-MB-231 & $\downarrow$ Cyclin D1,$\downarrow$ p65, $\downarrow$ PECAM- 1 & $\downarrow$ Angiogenesis, $\downarrow$ proliferation, $\uparrow$ apoptosis & 84 \\
\hline & MDA-MB-231 & $\uparrow \operatorname{miR} 181 \mathrm{~b}, \downarrow$ CXCL1,$\downarrow$ CXCL2 & $\begin{array}{l}\downarrow \text { Metastasis, } \downarrow \text { proliferation, } \downarrow \text { invasion, } \\
\uparrow \text { apoptosis }\end{array}$ & 284 \\
\hline & MDA-MB-231 \& MCF-7 & $\downarrow \mathrm{Bcl}-2, \uparrow \mathrm{Bax}$ & $\uparrow$ Apoptosis & 68 \\
\hline & MCF-7 \& T47D & $\begin{array}{l}\uparrow \mathrm{E} \text {-Cadherin/ } \beta \text {-catenin negative } \\
\text { feedback loop }\end{array}$ & $\downarrow$ Migration of bCSCs & 71 \\
\hline & MCF-7 & $\uparrow$ Nrf2 expression, $\downarrow$ Fen 1 expression & $\downarrow$ Proliferation & 70 \\
\hline & MCF-7 \& MDA-MB-231 & $\begin{array}{l}\downarrow N F-\kappa B-S n a i l \text { signalling pathway, } \\
\uparrow \text { E-cadherin, } \downarrow \text { vimentin }\end{array}$ & $\downarrow$ Invasion & 285 \\
\hline & $\begin{array}{l}\text { MCF-7/LCC2 \& MCF-7/ } \\
\text { LCC9 }\end{array}$ & $\begin{array}{l}\downarrow \text { NF- } \mathrm{BB}, \downarrow \text { Src }, \downarrow \text { FAK }, \downarrow \text { Akt }, \downarrow \text { mTOR, } \\
\downarrow \text { EZH } 2, \downarrow \text { Bcl- } 2, \downarrow \text { Bcl-xL }, \downarrow \text { cyclin D1 } \\
\downarrow \text { c-Myc }, \uparrow \text { ERK } 1 / 2\end{array}$ & $\begin{array}{l}\downarrow \text { Proliferation, } \uparrow \text { apoptosis, } \downarrow \text { endocrine } \\
\text { resistance, } \\
\uparrow \text { sensitization to tamoxifen }\end{array}$ & 286 \\
\hline & MCF-7 & $\downarrow$ uPA expression, $\downarrow$ NF- $\kappa \mathrm{B}$ & $\downarrow$ Adhesion, $\downarrow$ invasion & 82 \\
\hline & $\begin{array}{l}\text { MCF-7, MCF-10A, } \\
\text { MDA-MB-231, SK-BR-3h }\end{array}$ & $\begin{array}{l}\downarrow \text { HER-2 oncoprotein, } \downarrow \text { MAPK, } \\
\downarrow \text { p-Akt, } \downarrow \text { NF- } \mathrm{B}\end{array}$ & $\downarrow$ Proliferation, $\downarrow$ migration & 287 \\
\hline & MCF-7 \& MDA-MB-231 & $\downarrow$ DNMT1, $\downarrow$ RASSF1A methylation & $\uparrow$ RASSF1A & 74 \\
\hline & MDA-MB-231 & $\downarrow$ pERK1/2, $\downarrow$ pEGFR & $\uparrow$ Apoptosis, $\downarrow$ proliferation & 288 \\
\hline & MCF-7 & $\downarrow$ MAPK,$\downarrow$ PKC,$\downarrow$ NF- $\kappa \mathrm{B}$ & $\downarrow$ Invasion & 289 \\
\hline \multirow[t]{15}{*}{ EGCG } & In vivo & $\downarrow$ Ki-67 & $\downarrow$ Proliferation & 290 \\
\hline & $\mathrm{T}-47 \mathrm{D}$ & $\downarrow$ Telomerase, $\downarrow$ PI3K/AKT & $\uparrow$ Apoptosis & 92 \\
\hline & MCF-7 & $\downarrow \mathrm{Bcl}-2, \uparrow \mathrm{p} 53$ & $\uparrow$ Apoptosis, $\downarrow$ proliferation & 93 \\
\hline & MDA-MB-231 & $\downarrow \beta$-Catenin signalling pathway & $\downarrow$ Proliferation & 94 \\
\hline & $\mathrm{T}-47 \mathrm{D}$ & $\downarrow \mathrm{ER} \alpha$ protein levels & $\downarrow$ Proliferation & 291 \\
\hline & MCF-7/TAM & $\downarrow$ Nrf2 expression & $\downarrow$ Tamoxifen resistance & 100 \\
\hline & MCF-7/DOX & $\downarrow$ MMP-2, $\downarrow$ MMP-9 activity & $\downarrow$ Doxorubicin resistance & 101 \\
\hline & $\begin{array}{l}\text { MDA-MB-231 \& MDA-MB- } \\
436\end{array}$ & $\begin{array}{l}\downarrow \text { ER- } \alpha 36, \downarrow \text { MAPK/ERK, } \downarrow \text { EGFR, } \\
\downarrow \text { PI3K/AKT }\end{array}$ & $\downarrow$ Proliferation, $\downarrow$ bCSCs growth & 98 \\
\hline & MCF-7, T-47D \& SK-BR-3 & $\begin{array}{l}\downarrow \text { Hsp90/PR-B/HDAC interactions, } \\
\uparrow \mathrm{p} 38 / \mathrm{CK} 2, \downarrow \mathrm{ER} \alpha\end{array}$ & $\downarrow$ Proliferation, $\downarrow$ colonization & 96 \\
\hline & MCF-7 \& MDA-MB-231 & $\begin{array}{l}\downarrow \text { H3K9/18 acetylation, } \downarrow \text { EZH } 2, \\
\downarrow \text { class I HDAC, } \downarrow \text { TIMP-3, } \\
\downarrow \text { H3K2 trimethylation }\end{array}$ & $\downarrow$ Progression \& invasion & 292 \\
\hline & SUM-149 \& SUM-190 & $\begin{array}{l}\downarrow \text { ALDH }+ \text { cells, } \downarrow \text { VEGF-D, } \downarrow \text { cyclin D1, } \\
\downarrow \text { RhoC, } \downarrow \text { FN1, } \downarrow \text { E-cadherin, } \downarrow \text { Bcl-XL, } \\
\downarrow \text { VIM, } \uparrow \text { c-PARP }, \uparrow \text { cleaved caspase-3 }\end{array}$ & $\begin{array}{l}\downarrow \text { Proliferation, } \downarrow \text { migration, } \\
\downarrow \text { invasion, } \uparrow \text { apoptosis, } \\
\downarrow \text { lymphangiogenesis, } \downarrow \text { tumorsphere } \\
\text { formation }\end{array}$ & 293 \\
\hline & Hs578T & $\downarrow$ VEGF & $\begin{array}{l}\downarrow \text { Proliferation, } \downarrow \text { migration \& } \\
\text { invasion }\end{array}$ & 294 \\
\hline & MCF-7 & $\downarrow$ VEGF,$\downarrow$ HIF- $1 \alpha$ & $\downarrow$ Proliferation & 295 \\
\hline & 4T1 \& RAW264.7 & $\begin{array}{l}\uparrow \operatorname{miR}-16, \downarrow \text { IKK } \alpha, \downarrow \text { CSF- } 1, \uparrow \text { IL- } 6, \downarrow \text { CCL- } 2, \\
\uparrow \text { TGF- } \beta, \uparrow \text { TNF- } \alpha\end{array}$ & $\begin{array}{l}\downarrow \text { Tumor growth, } \downarrow \text { TAM } \\
\text { infiltration, } \downarrow \text { M } 2 \text { polarization }\end{array}$ & 296 \\
\hline & MCF-7 \& MDA-MB-231 & $\uparrow \mathrm{p} 21^{\mathrm{WAF} 1}, \downarrow$ DNMT1,$\downarrow$ HDAC $1, \downarrow$ MeCP2 & $\downarrow$ Cell viability & 297 \\
\hline
\end{tabular}


Table 2 (Contd.)

\begin{tabular}{|c|c|c|c|c|}
\hline Phytochemical & Cell line/model & Proposed mechanism & Effect & Ref. \\
\hline \multirow[t]{12}{*}{ Genistein } & Sprague-Dawley rats & $\begin{array}{l}\downarrow \text { UPR }, \downarrow \text { GRP78, } \downarrow \text { IRE1 } \alpha, \downarrow \text { Beclin- } 1, \\
\downarrow \text { ATF } 4, \downarrow \text { TGF } \beta, \downarrow \text { Fox } \rho 3, \uparrow \text { CD8a }\end{array}$ & $\downarrow$ Tamoxifen resistance, $\downarrow$ recurrence & 110 \\
\hline & MCF-7-C3 \& T-47D & $\downarrow \mathrm{CIP} 2 \mathrm{~A}, \downarrow$ E2F1 & $\uparrow$ Apoptosis, $\downarrow$ proliferation & 106 \\
\hline & MCF-7 & $\uparrow \operatorname{miR}-23 \mathrm{~b}$ & $\uparrow$ Cytotoxicity & 111 \\
\hline & MDA-MB-231 \& MCF-7 & $\begin{array}{l}\downarrow \text { DNMTI, } \uparrow \text { ATM, } \uparrow \text { PTEN, } \uparrow \text { APC, } \\
\uparrow \text { SERPINB5 }\end{array}$ & $\downarrow$ Cell viability & 108 \\
\hline & MCF-7/Adr & $\downarrow$ HER2/neu & $\begin{array}{l}\uparrow \text { Apoptosis, } \downarrow \text { doxorubicin } \\
\text { resistance }\end{array}$ & 298 \\
\hline & MCF-7 \& 3T3-L1 & $\downarrow \mathrm{ER} \alpha, \downarrow$ cyclin D1, $\downarrow$ Bcl-2, $\uparrow$ Bax & $\downarrow$ Proliferation, $\uparrow$ apoptosis & 103 \\
\hline & MCF-7 & $\downarrow$ Hedgehog, $\downarrow$ Gli1 & $\downarrow$ bCSCs & 113 \\
\hline & MDA-MB-231 \& MCF-7 & $\begin{array}{l}\uparrow \mathrm{ATM}, \uparrow \mathrm{Chk} 2, \uparrow \mathrm{Cdc} 25 \mathrm{C}, \uparrow \mathrm{Cdc} 2, \\
\uparrow \mathrm{Bax}, \uparrow \mathrm{p} 53, \downarrow \text { Bcl-2}, \downarrow \text { Rad51 }\end{array}$ & $\uparrow$ Radiosensitivity, $\uparrow$ apoptosis & 299 \\
\hline & MCF-7, SK-BR-3 \& ZR-75-1 & $\downarrow \mathrm{ER} \alpha, \downarrow$ c-erbB-2 expression & $\downarrow$ Proliferation & 105 \\
\hline & MDA-MB-231 & $\downarrow \mathrm{pERK} 1 / 2, \uparrow \mathrm{Bax} / \mathrm{Bcl}-2$ ratio & $\uparrow$ Apoptosis, $\downarrow$ proliferation & 300 \\
\hline & MDA-MB-231 & $\downarrow$ NF- $\kappa \mathrm{B}, \downarrow$ cyclin B1,$\downarrow$ Bcl-2, $\downarrow$ Bcl-xL & $\downarrow$ Proliferation, $\uparrow$ apoptosis & 301 \\
\hline & MCF-7 \& UACC-3199 & $\begin{array}{l}\downarrow \text { DNMT- } 1, \downarrow \text { cyclin D1, } \uparrow p 53, \\
\uparrow \text { BRCA- } 1, \downarrow \text { CpG methylation, } \uparrow \text { CYP1A1 }\end{array}$ & $\downarrow$ Proliferation & 40 \\
\hline \multirow[t]{12}{*}{ Resveratrol } & MDA-MB-231 \& MCF-7 & $\uparrow$ ATP2A3, $\downarrow$ Bcl-2, $\downarrow$ Ki67, $\uparrow$ Bcl-2L11 (BIM) & $\uparrow$ Apoptosis, $\downarrow$ proliferation & 114 \\
\hline & T47-D & $\downarrow \mathrm{p} 53, \downarrow \mathrm{ER} \alpha$ & $\downarrow$ Proliferation & 302 \\
\hline & MDA-MB-231 & $\downarrow$ AURKA, $\downarrow$ PLK1, $\downarrow$ cyclin D1, $\downarrow$ cyclin B1 & $\begin{array}{l}\downarrow \text { Cell cycle progression, } \\
\uparrow \text { apoptosis, } \downarrow \text { viability }\end{array}$ & 117 \\
\hline & $\begin{array}{l}\text { MDA-MB-231 \& MDA-MB- } \\
468\end{array}$ & $\downarrow$ YAP, $\downarrow$ RhoA & $\downarrow$ Invasion & 116 \\
\hline & MDA-MB-231 \& MCF-7 & $\begin{array}{l}\downarrow \text { XIAP, } \downarrow \text { Bcl-2, } \uparrow \text { CASP-8, } \uparrow \text { CASP-9, } \\
\text { modulation of miR-125b-5p, miR-409-3p, } \\
\text { miR-200c-3, miR-542-3p \& miR-122-5p }\end{array}$ & $\uparrow$ Apoptosis & 128 \\
\hline & MCF-7/DOX & $\downarrow$ MDR-1, $\downarrow$ P-glycoprotein & $\downarrow$ Doxorubicin resistance, $\downarrow$ proliferation & 124 \\
\hline & MCF-7, SUM159 & $\downarrow$ Wnt, $\downarrow \beta$-catenin & $\begin{array}{l}\downarrow \text { bCSCs proliferation, } \\
\uparrow \text { autophagy, } \downarrow \text { mammospheres }\end{array}$ & 303 \\
\hline & SKBR-3 & $\begin{array}{l}\downarrow \text { FASN }, \downarrow \text { HER } 2, \uparrow \text { PEA3 }, \downarrow \text { p-Akt, } \uparrow \text { PTEN }, \\
\downarrow \text { PI3K, } \downarrow \text { Akt }, \downarrow \text { mTOR }\end{array}$ & $\downarrow$ Proliferation, $\uparrow$ apoptosis & 115 \\
\hline & MCF-7 & $\begin{array}{l}\downarrow \text { PFK activity, } \downarrow \text { glucose consumption, } \\
\downarrow \text { ATP content }\end{array}$ & $\downarrow$ Cell viability & 304 \\
\hline & MCF-7/TR & $\downarrow$ TGF- $\beta, \downarrow$ Smad cascade, $\downarrow$ EMT & $\downarrow$ Tamoxifen resistance, $\uparrow$ apoptosis & 126 \\
\hline & MCF-7 & $\downarrow$ HSP27 & $\uparrow$ Sensitization to doxorubicin & 125 \\
\hline & MCF-7 \& MDA-MB-231 & $\begin{array}{l}\uparrow \mathrm{p} 53, \downarrow \text { procaspase } 8, \uparrow \text { CASP-7, } \uparrow \text { CASP-9, } \\
\uparrow \text { p-Chk2, } \downarrow \text { cyclin A, } \downarrow \text { Thr }^{160}- \\
\text { phosphorylated CDK2, } \downarrow \text { CDK7, } \uparrow \text { cell cycle arrest }\end{array}$ & $\uparrow$ Sensitization to melphalan & 127 \\
\hline \multirow[t]{11}{*}{ Quercetin } & $\begin{array}{l}\text { MDA-MB-231 \& MDA-MB- } \\
157\end{array}$ & $\downarrow$ FASN,$\downarrow \beta$-catenin, $\downarrow$ Bcl-2, $\uparrow$ caspase-3 & $\uparrow$ Apoptosis, $\downarrow$ proliferation & 44 \\
\hline & MCF-7 & $\downarrow \mathrm{Bcl}-2, \uparrow \mathrm{Bax}$ & $\uparrow$ Apoptosis, $\uparrow$ necroptosis & 130 \\
\hline & MCF-7 & $\begin{array}{l}\downarrow \text { VEGF, } \downarrow \text { VEGFR } 2, \downarrow \text { NFATc } 3, \downarrow \text { calcineurin } \\
\text { pathway }\end{array}$ & $\downarrow$ Angiogenesis & 137 \\
\hline & MCF-7Ca/TAM-R & $\downarrow$ Her- $2, \uparrow \mathrm{ER} \alpha$ & $\begin{array}{l}\downarrow \text { Proliferation, } \uparrow \text { apoptosis, } \downarrow \text { tamoxifen } \\
\text { resistance }\end{array}$ & 134 \\
\hline & $\begin{array}{l}\text { MDA-MB-231 \& MDA-MB- } \\
468\end{array}$ & $\begin{array}{l}\uparrow \text { E-Cadherin, } \downarrow \text { vimentin, } \downarrow \text { c-Myc, } \\
\downarrow \text { cyclin D1 }\end{array}$ & $\downarrow$ Metastasis, $\downarrow$ proliferation & 136 \\
\hline & MCF-7 & $\downarrow$ Twist, $\downarrow$ p38MAPK, $\downarrow$ cyclin D1, $\downarrow$ p21 & $\uparrow$ Apoptosis & 131 \\
\hline & MCF-7 & $\downarrow$ Proteasome, $\uparrow$ CASP- $3, \uparrow$ CASP-7 & $\downarrow$ Proliferation, $\uparrow$ apoptosis & 129 \\
\hline & MCF-7 \& MDA-MB-231 & $\uparrow \operatorname{miR}-146 \mathrm{a}, \downarrow$ EGFR, $\uparrow \mathrm{Bax}, \uparrow \mathrm{CASP}-3$ & $\begin{array}{l}\downarrow \text { Proliferation, } \downarrow \text { invasion, } \\
\uparrow \text { apoptosis }\end{array}$ & 138 \\
\hline & MCF-7 & $\downarrow$ Survivin & $\downarrow$ Proliferation & 132 \\
\hline & MCF-7/TR & $\downarrow$ Cyclin E2 & $\uparrow$ Sensitization to tamoxifen & 305 \\
\hline & MCF-7 & $\downarrow \mathrm{Bcl}-2, \uparrow \mathrm{Bax}$ & $\downarrow$ Proliferation, $\uparrow$ apoptosis & 306 \\
\hline \multirow[t]{6}{*}{ Kaempferol } & MCF-7 & $\begin{array}{l}\downarrow \text { pIRS- } 1, \downarrow \text { pAkt }, \downarrow \text { pMEK } 1 / 2, \text { pERK } 1 / 2, \\
\downarrow \text { cyclin D } 1, \downarrow \text { cyclin E }, \uparrow \text { p21, } \downarrow \text { cathepsin D }\end{array}$ & $\downarrow$ Proliferation, $\downarrow$ metastasis, $\uparrow$ apoptosis & 243 \\
\hline & MCF-7 & $\downarrow$ Bcl-2, $\uparrow$ Bax,$\uparrow$ PARP cleavage & $\uparrow$ Apoptosis & 242 \\
\hline & MCF-7 & $\begin{array}{l}\downarrow \text { Cathepsin B }, \downarrow \text { cathepsin } \mathrm{D}, \downarrow \text { N-cadherin, } \\
\downarrow \text { snail, } \downarrow \text { slug, } \uparrow \text { E-cadherin }\end{array}$ & $\begin{array}{l}\downarrow \text { Proliferation, } \downarrow \text { migration, } \\
\downarrow \text { invasion, } \downarrow \text { metastasis }\end{array}$ & 307 \\
\hline & $\begin{array}{l}\text { MDA-MB-231 \& MDA-MB- } \\
453\end{array}$ & $\downarrow$ RhoA, $\downarrow$ Rac1 & $\downarrow$ Migration, $\downarrow$ invasion & 42 \\
\hline & MCF-7 & $\begin{array}{l}\uparrow \text { E-cadherin, } \downarrow \text { MMP-9, } \downarrow \text { MMP-2, } \\
\downarrow \text { cathepsin B } \downarrow \text { cathepsin D, } \downarrow \text { N-cadherin, } \\
\downarrow \text { snail, } \downarrow \text { slug }\end{array}$ & $\downarrow$ Metastasis & 244 \\
\hline & MCF-7 & $\downarrow$ GLUT1, $\downarrow$ MCT1 & $\downarrow$ Proliferation, $\uparrow$ cytotoxicity & 241 \\
\hline
\end{tabular}


Table 2 (Contd.)

\begin{tabular}{|c|c|c|c|c|}
\hline Phytochemical & Cell line/model & Proposed mechanism & Effect & Ref. \\
\hline \multirow[t]{8}{*}{ Apigenin } & MDA-MB-231 & $\downarrow$ Cyclin A, $\downarrow$ cyclin B,$\downarrow$ CDK1, $\uparrow \mathrm{p} 21^{\mathrm{WAF} 1 / \mathrm{CIP} 1}$ & $\downarrow$ Proliferation & 176 \\
\hline & MCF-7/ADR & $\downarrow$ MDR1, $\downarrow$ P-gp,$\downarrow$ p-STAT3, $\downarrow$ VEGF,$\downarrow$ MMP-9 & $\begin{array}{l}\uparrow \text { Apoptosis, } \downarrow \text { colonization, } \downarrow \text { adriamycin } \\
\text { resistance }\end{array}$ & 173 \\
\hline & MDA-MB-231 & $\begin{array}{l}\downarrow \mathrm{TNF} \alpha, \downarrow \mathrm{CCL} 2, \downarrow \text { GMCSF }, \downarrow \text { IL-1 } \alpha, \downarrow \text { IL-6, } \\
\downarrow \text { IKBKe }\end{array}$ & $\downarrow$ Pro-inflammatory cytokines & 163 \\
\hline & MDA-MB-231 \& ZR75.1 & $\begin{array}{l}\downarrow \text { CXCL10, } \downarrow \text { IL-6, } \downarrow \text { IL-1 } \alpha, \downarrow \text { IRAK1, } \downarrow \text { IRAK4 }, \\
\downarrow \text { NF- } \kappa \mathrm{B}, \downarrow \text { p38-MAPK }, \downarrow \text { IP10 }\end{array}$ & $\begin{array}{l}\downarrow \text { Impact of senescent cells } \\
\text { on breast cancer cells, } \downarrow \text { fibroblast } \\
\text { proliferation }\end{array}$ & 308 \\
\hline & BT- 474 & $\begin{array}{l}\downarrow \text { p-JAK1, } \downarrow \text { p-JAK2 }, \downarrow \text { p-STAT3, } \downarrow \text { VEGF, } \\
\uparrow \text { c-CASP-8, } \uparrow \text { c-CASP-3 }\end{array}$ & $\uparrow$ Apoptosis, $\downarrow$ proliferation, $\downarrow$ colonization & 174 \\
\hline & MDA-MB-468 & $\downarrow$ Akt & $\downarrow$ Proliferation & 177 \\
\hline & MDA-MB-231 \& T47D & $\uparrow \mathrm{CASP}-3, \uparrow \mathrm{c}-\mathrm{PARP}, \uparrow \mathrm{Bax}, \downarrow \mathrm{Bcl}-2, \uparrow \mathrm{LC} 3-\mathrm{II}$ & $\begin{array}{l}\uparrow \text { Apoptosis, } \downarrow \text { proliferation, } \downarrow \text { colonization, } \\
\uparrow \text { autophagy }\end{array}$ & 309 \\
\hline & MCF-7/HER2 \& MCF-7 vec & 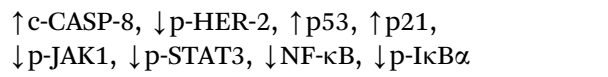 & $\uparrow$ Apoptosis, $\downarrow$ proliferation & 175 \\
\hline \multirow[t]{14}{*}{ Silibinin } & MCF-7 \& T47D & $\downarrow$ miR-21, $\downarrow$ Bcl-2 & $\uparrow$ Apoptosis & 149 \\
\hline & MCF-7 & $\downarrow$ Maspin, $\downarrow$ ER $\alpha$ & $\downarrow$ Proliferation & 310 \\
\hline & T47D & $\downarrow$ hTERT, $\downarrow$ cyclin D1 & $\downarrow$ Proliferation & 147 \\
\hline & MCF-7 & $\downarrow \mathrm{Bcl}-2, \uparrow \mathrm{p} 53, \uparrow \mathrm{Bax}, \uparrow \mathrm{BRCA} 1, \uparrow \mathrm{ATM}$ & $\uparrow$ Apoptosis, $\downarrow$ proliferation & 145 \\
\hline & MCF-7 \& T47D & $\uparrow$ PTEN, $\uparrow \mathrm{p} 21, \downarrow \mathrm{Bcl}-2, \uparrow \mathrm{p} 27$ & $\begin{array}{l}\downarrow \text { Proliferation, } \uparrow \text { apoptosis, } \\
\uparrow \text { necrosis }\end{array}$ & 311 \\
\hline & MCF-7 & $\downarrow$ miR-21, $\downarrow$ miR-155, $\uparrow$ CASP-9, $\uparrow$ BID & $\uparrow$ Apoptosis, $\downarrow$ proliferation & 148 \\
\hline & MCF-7 & $\begin{array}{l}\downarrow \mathrm{ER} \alpha, \downarrow \text { Akt }, \downarrow \mathrm{mTOR}, \downarrow \mathrm{ERK}, \uparrow \mathrm{CASP}-6, \\
\uparrow \mathrm{p} 53, \downarrow \text { APAF- } 1, \downarrow \mathrm{p} 62, \uparrow \mathrm{Bax}, \downarrow \mathrm{Bcl}-2, \\
\uparrow \mathrm{LC} 3-\mathrm{I} \text { to LC3-II }\end{array}$ & $\uparrow$ Apoptosis, $\uparrow$ autophagy & 151 \\
\hline & MCF-7 & $\begin{array}{l}\uparrow \text { Atg12-Atg5, } \uparrow \text { LC3-1 to LC3II, } \uparrow \text { Beclin-1, } \\
\downarrow \text { Bcl-2, } \uparrow \text { BNIP3, } \uparrow \text { ROS }\end{array}$ & $\uparrow$ Autophagy & 150 \\
\hline & MCF-7 & $\uparrow \mathrm{p} 53, \uparrow \mathrm{p} 21, \uparrow \mathrm{BRCA} 1, \uparrow \mathrm{Bak}, \uparrow \mathrm{ATM}, \downarrow \mathrm{Bcl}-\mathrm{xl}$ & $\uparrow$ Apoptosis, $\downarrow$ proliferation & 143 \\
\hline & MCF-7 \& MDA-MB-231 & $\downarrow$ ERK,$\downarrow$ Akt,$\downarrow$ Notch- 1 & $\uparrow$ Apoptosis & 312 \\
\hline & SKBR3 & $\downarrow N F-\kappa B$ & $\uparrow$ Apoptosis, $\downarrow$ proliferation & 142 \\
\hline & MDA-MB-468 & $\downarrow$ EGFR,$\downarrow$ VEGF,$\downarrow$ COX-2, $\downarrow$ MMP-9 & $\begin{array}{l}\downarrow \text { Metastasis, } \downarrow \text { infiltration, } \\
\downarrow \text { tumor volume }\end{array}$ & 144 \\
\hline & MCF-7 & $\downarrow$ MMP-9, $\downarrow$ MEK,$\downarrow$ ERK & $\downarrow$ Migration & 141 \\
\hline & MDA-MB-231 \& T47D & $\downarrow$ Wnt, $\downarrow \beta$-catenin, $\downarrow$ LRP6 & $\downarrow$ Proliferation & 313 \\
\hline \multirow[t]{5}{*}{ Pterostilbene } & MDA-MB-468 & $\begin{array}{l}\uparrow \mathrm{ERK} 1 / 2, \downarrow \text { cyclin D1, } \uparrow \mathrm{p} 21, \downarrow \text { Akt }, \downarrow \mathrm{mTOR}, \\
\uparrow \mathrm{Bax}\end{array}$ & $\uparrow$ Apoptosis, $\downarrow$ proliferation & 189 \\
\hline & $\begin{array}{l}\text { MCF, MDA-MB-231 \& } \\
\text { Hs578t }\end{array}$ & $\begin{array}{l}\uparrow \text { E-cadherin, } \downarrow \text { vimentin, } \downarrow \text { snail, } \downarrow \text { slug, } \downarrow \text { ZEB } 1, \\
\uparrow \text { miR-205, } \downarrow \text { Src/Fak }\end{array}$ & $\downarrow$ Metastasis & 191 \\
\hline & MCF-7 & $\begin{array}{l}\downarrow \text { CD44, } \uparrow \beta \text {-catenin, } \downarrow \text { hedgehog, } \downarrow \text { Akt, } \downarrow \text { GSK3 } \beta \\
\text { signalling, } \downarrow \text { cyclin D1, } \downarrow \text { c-Myc }\end{array}$ & $\downarrow$ bCSCs, $\downarrow$ mammospheres & 195 \\
\hline & MCF-7 \& MDA-MB-231 & $\downarrow N F \kappa \mathrm{B}, \downarrow$ vimentin, $\downarrow$ Twist1, $\uparrow$ E-cadherin & $\downarrow$ bCSCs, $\downarrow$ metastasis & 196 \\
\hline & MDA-MB-231 & $\begin{array}{l}\downarrow \text { MMP- } 2, \downarrow \text { MMP-9, } \downarrow \text { cortactin, } \downarrow \text { c-Src kinase, } \\
\downarrow \text { MT1-MMP }\end{array}$ & $\downarrow$ Metastasis & 314 \\
\hline \multirow[t]{6}{*}{ Sulforaphane } & SUM-149 \& SUM-159 & $\downarrow N F-\kappa B$ p65 subunit, $\downarrow$ p52 & $\downarrow$ bCSCs, $\downarrow$ mammospheres, $\downarrow$ proliferation & 204 \\
\hline & $\begin{array}{l}\text { MCF-7, MDA-MB-231 \& SK- } \\
\text { BR-3 }\end{array}$ & $\begin{array}{l}\downarrow \text { DNMT1, } \downarrow \text { DNMT3B }, \uparrow \mathrm{p} 21, \uparrow \mathrm{p} 27, \downarrow \text { miR92b, } \\
\downarrow \text { miR-23b } \downarrow \text { miR-381, } \downarrow \text { miR-382, } \downarrow \text { Akt }, \downarrow \text { AMPK, } \\
\downarrow \text { ATP }\end{array}$ & $\begin{array}{l}\uparrow \text { Cellular senescence, } \uparrow \text { apoptosis, } \\
\uparrow \text { autophagy }\end{array}$ & 210 \\
\hline & MCF-7 \& MDA-MB-231 & $\downarrow$ Akt,$\downarrow$ NF- $\kappa \mathrm{B}, \downarrow \mathrm{Bcl}-2$ & $\uparrow$ Apoptosis, $\uparrow$ sensitization to paclitaxel & 205 \\
\hline & MCF10DCIS.com & $\downarrow$ TNF- $\alpha, \downarrow$ MMP- $2, \downarrow$ MMP-9, $\downarrow$ MMP-13 & $\downarrow$ Migration, $\downarrow$ invasion & 206 \\
\hline & MCF-7 & $\downarrow \mathrm{Bcl}-2, \downarrow \mathrm{COX}-2$ & $\uparrow$ Apoptosis, $\downarrow$ proliferation & 315 \\
\hline & MCF-7 & $\downarrow$ MMP-9, $\downarrow$ NF- $\mathrm{B}$ & $\downarrow$ Invasion & 207 \\
\hline \multirow[t]{9}{*}{ Luteolin } & MDA-MB-231 (4175) LM2 & $\downarrow$ VEGF & $\downarrow$ Angiogenesis, $\downarrow$ lung metastasis & 274 \\
\hline & MCF-7 \& MDA-MB-231 & $\downarrow$ p-EGFR, $\downarrow$ p-STAT3, $\downarrow$ p-AKT, $\downarrow$ p-ERK1/2 & $\downarrow$ Proliferation & 316 \\
\hline & BT-474 \& T47D & $\downarrow$ VEGF $, \downarrow \mathrm{CD} 44, \downarrow$ ALDH & $\downarrow$ Angiogenesis, $\downarrow$ proliferation, $\uparrow$ apoptosis & 317 \\
\hline & $\begin{array}{l}\text { MDA-MB-231, BT5-49 \& } \\
\text { female nude mice }\end{array}$ & $\downarrow$ Vimentin, $\downarrow$ slug, $\downarrow \beta$-catenin & $\downarrow$ Lung metastasis & 275 \\
\hline & MDA-MB-231 & $\begin{array}{l}\downarrow \text { Notch signalling, } \downarrow \text { VEGF }, \downarrow \text { cyclin D1, } \\
\downarrow \text { MMP- } 2, \downarrow \text { MMP-9, } \downarrow \text { Hes- } 1\end{array}$ & $\begin{array}{l}\downarrow \text { Migration, } \downarrow \text { angiogenesis, } \\
\downarrow \text { cell survival }\end{array}$ & 318 \\
\hline & MCF-7 (TAM-R) & $\downarrow$ Cyclin E2 & $\downarrow$ Tamoxifen resistance & 278 \\
\hline & MDA-MB-231 & $\begin{array}{l}\downarrow \text { AKT }, \downarrow \text { cyclin A, } \downarrow \text { PLK1 }, \downarrow \text { CDC } 2, \downarrow \text { CDK2, } \\
\downarrow \text { cyclin } \mathrm{B}_{1}, \downarrow \text { Bcl-xL }, \uparrow \text { p } 21, \uparrow \text { Bax }, \downarrow \text { EGFR }, \\
\downarrow \text { MAPK }\end{array}$ & $\uparrow$ Apoptosis, $\downarrow$ proliferation & 319 \\
\hline & $\begin{array}{l}\text { MDA-MB-231, } \\
\text { MCF-7 \& SK-BR-3 }\end{array}$ & $\uparrow \mathrm{ERK}, \uparrow \mathrm{p} 38, \uparrow \mathrm{CASP}-3, \uparrow \mathrm{c}-\mathrm{PARP}$ & $\uparrow$ Apoptosis & 320 \\
\hline & MCF-7 & $\downarrow$ IGF-1, $\downarrow$ Akt,$\downarrow$ ER $\alpha$ & $\uparrow$ Apoptosis, $\downarrow$ proliferation & 321 \\
\hline
\end{tabular}


Table 2 (Contd.)

\begin{tabular}{|c|c|c|c|c|}
\hline Phytochemical & Cell line/model & Proposed mechanism & Effect & Ref. \\
\hline \multirow[t]{8}{*}{ Naringenin } & MCF-7 (Tam-R) & $\downarrow$ MAPK, CASP-7 & $\uparrow$ Apoptosis, $\downarrow$ proliferation & 322 \\
\hline & $4 \mathrm{~T} 1$ & $\downarrow$ TGF- $\beta 1, \downarrow$ PKC & $\downarrow$ Pulmonary metastasis & 323 \\
\hline & MDA-MB-231 & $\downarrow$ Integrin $\beta 3, \downarrow$ MMP- $2, \downarrow$ MMP-9 & $\downarrow$ Migration \& invasion & 324 \\
\hline & НTB26 \& HTB132 & 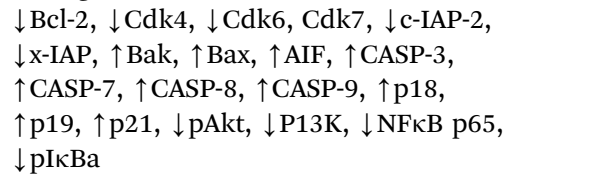 & $\begin{array}{l}\uparrow \text { Apoptosis, } \downarrow \text { proliferation, } \\
\uparrow \text { chemo-sensitization }\end{array}$ & 325 \\
\hline & E0771 & $\uparrow \mathrm{AMPK}, \downarrow$ Bcl-2, $\downarrow$ cyclin D1 & $\uparrow$ Apoptosis, $\downarrow$ proliferation & 326 \\
\hline & MDA-MB-231 & $\downarrow$ Survivin, $\uparrow \mathrm{p} 21, \beta$-catenin & $\downarrow$ Proliferation, $\uparrow$ apoptosis & 339 \\
\hline & MCF-7 & $\begin{array}{l}\downarrow \mathrm{P} 13 \mathrm{~K}, \downarrow \text { MAPK }, \downarrow \text { ERK } 1 / 2, \downarrow \text { AKT, } \\
\uparrow \text { CASP- } 7, \uparrow \text { CASP- } 9\end{array}$ & $\uparrow$ Apoptosis, $\downarrow$ proliferation & 328 \\
\hline & $\begin{array}{l}\text { MCF-7, T47D \& MDA-MB- } \\
231\end{array}$ & $\uparrow \mathrm{CASP}-3, \downarrow \mathrm{AKT}, \uparrow \mathrm{p} 38$ & $\uparrow$ Apoptosis, $\downarrow$ proliferation & 329 \\
\hline \multirow[t]{5}{*}{$\alpha$-Mangostin } & $\begin{array}{l}\text { T47D, MDA-MB-468, } \\
\text { SKBR3 \&AU565 }\end{array}$ & $\begin{array}{l}\downarrow \mathrm{Bcl}-2, \downarrow \mathrm{Mcl}-1, \downarrow \mathrm{P} 13 \mathrm{~K}, \downarrow \mathrm{ERK} 1 / 2, \downarrow \mathrm{ER} \alpha, \\
\downarrow \text { HER2, } \downarrow \text { Akt }, \downarrow \mathrm{ERK} 1 / 2, \uparrow \mathrm{p}-\mathrm{p} 38, \uparrow \mathrm{p}-\mathrm{JNK} 1 / 2, \\
\downarrow \mathrm{MAPK}\end{array}$ & $\uparrow$ Apoptosis, $\downarrow$ proliferation, $\downarrow$ colonization & 251 \\
\hline & MCF-7 \& MDA-MB-231 & $\begin{array}{l}\uparrow \mathrm{p} 53, \uparrow \mathrm{Bax}, \uparrow \text { PARP cleavage, } \downarrow \text { Bcl- } 2, \downarrow \text { Bid } \\
\downarrow \mathrm{pS} 2, \downarrow \mathrm{ER} \alpha, \uparrow \text { CASP-7, } \uparrow \text { CASP-8, } \uparrow \text { CASP-9 }\end{array}$ & $\uparrow$ Apoptosis, $\downarrow$ proliferation & 254 \\
\hline & MCF-7 \& MDA-MB-231 & $\begin{array}{l}\downarrow \text { FASN }, \downarrow \text { FAK, } \downarrow \text { pAKT }, \uparrow \text { Bcl- } 2, \downarrow \text { Bax } \\
\uparrow \text { p-ERK1 } / 2\end{array}$ & $\uparrow$ Apoptosis & 253 \\
\hline & MDA-MB-231 & $\uparrow$ CASP-3 & $\uparrow$ Apoptosis, $\downarrow$ proliferation & 252 \\
\hline & MDA-MB-231 & $\begin{array}{l}\uparrow \mathrm{p} 21^{\mathrm{cip} 1}, \uparrow \mathrm{CASP}-3, \uparrow \mathrm{CASP}-8, \uparrow \mathrm{CASP}-9, \\
\uparrow \mathrm{CHEK} 2, \downarrow \mathrm{CDKs}, \downarrow \text { PCNA }, \downarrow \text { cdc }(\mathrm{s})\end{array}$ & $\uparrow$ Apoptosis & 255 \\
\hline \multirow[t]{9}{*}{ Thymoquinone } & MCF-7, T47D \& EMT6/p & $\downarrow$ VEGF, $\uparrow$ IFN- $\gamma, \uparrow$ IL-4 & $\downarrow$ Angiogenesis, $\uparrow$ apoptosis, $\downarrow$ proliferation & 118 \\
\hline & MCF-7 & $\begin{array}{l}\downarrow \text { hsa04310 (Wnt), } \uparrow \text { hsa04115 (p53), } \\
\downarrow \text { hsa04151 (P13K/AKT), } \downarrow \text { hsa04010 (MAPK) }\end{array}$ & $\uparrow$ Apoptosis & 153 \\
\hline & MCF-7 & $\uparrow \mathrm{p} 53$ & $\uparrow$ Apoptosis & 331 \\
\hline & EMT6/p & $\downarrow$ VEGF,$\uparrow$ IFN- $\gamma, \downarrow$ IL- $4, \downarrow$ AST,$\downarrow$ ALT & $\begin{array}{l}\uparrow \text { Apoptosis, } \downarrow \text { proliferation, } \\
\uparrow \text { necrosis, } \downarrow \text { angiogenesis }\end{array}$ & 158 \\
\hline & MCF-7 & $\begin{array}{l}\uparrow \text { PTPRR }, \downarrow \text { MAPK }, \downarrow \text { p38-MAPK, } \uparrow \text { TGF- } \beta, \\
\downarrow \text { TP53, } \downarrow \text { Bcl-2, } \downarrow \text { CARD16, } \downarrow \text { EGF-EGFR } \downarrow \text { GPCR }\end{array}$ & $\uparrow$ Apoptosis & 152 \\
\hline & MCF-7 & $\uparrow$ Bax, $\uparrow \mathrm{p} 21, \uparrow$ Maspin, $\downarrow$ Bcl-2, $\downarrow$ HDAC & $\uparrow$ Apoptosis, $\downarrow$ proliferation, $\downarrow$ migration & 155 \\
\hline & 4T1, PMEF \& Balb/c mice & $\begin{array}{l}\uparrow \text { BRCA1, } \uparrow \text { p21, } \uparrow \text { HIC } 1, \uparrow \text { CASP-3, } \uparrow \text { CASP-7, } \\
\uparrow \text { CASP- } 12, \uparrow \text { PARP }, \downarrow \text { p65, } \downarrow \text { p-Akt1 }\end{array}$ & $\uparrow$ Apoptosis, $\downarrow$ proliferation, $\downarrow$ migration & 157 \\
\hline & MCF7 \& MDA-MB-231 & $\begin{array}{l}\uparrow \text { TGF- } \beta, \uparrow \text { E-cadherin, } \uparrow \text { cytokeratin } 19, \\
\downarrow \text { MMP-2, } \downarrow \text { MMP-9, } \downarrow \text { integrin } \alpha \mathrm{V}, \downarrow \text { snail, } \\
\downarrow \text { Twist, } \downarrow \text { Smad2, } \downarrow \text { NF- } \mathrm{B}\end{array}$ & $\begin{array}{l}\uparrow \text { Apoptosis, } \downarrow \text { proliferation, } \downarrow \text { migration, } \\
\downarrow \text { invasion, } \\
\downarrow \text { colonization, } \uparrow \text { sensitization to radiation }\end{array}$ & 154 \\
\hline & $\begin{array}{l}\text { MCF-7, T47D, MDA-MB- } \\
231 \text { \& } \\
\text { MDA-MB-468 }\end{array}$ & $\begin{array}{l}\downarrow \text { Cyclin D1, } \downarrow \text { cyclin E }, \downarrow \text { p27, } \downarrow \text { survivin, } \\
\downarrow \text { Bcl-xl, } \downarrow \text { Bcl- } 2, \uparrow \text { Bax }, \uparrow \text { PARP }, \uparrow \text { procaspase-3, } \\
\uparrow \text { Cyt c }, \downarrow \text { Akt }, \uparrow \text { PTEN }, \downarrow \text { PDK } 1\end{array}$ & $\begin{array}{l}\uparrow \text { Apoptosis, } \downarrow \text { proliferation, } \\
\downarrow \text { viability }\end{array}$ & 332 \\
\hline \multirow[t]{9}{*}{ Isoliquiritigenin } & $\begin{array}{l}\text { MCF-7, MDA-MB-231, 4T1, } \\
\text { BT474, MCF-10A }\end{array}$ & $\begin{array}{l}\downarrow \text { miR-374a, } \uparrow \text { PTEN, } \downarrow \text { Akt, } \uparrow \text { Bax, } \downarrow \text { Bcl- } 2, \\
\uparrow \text { c-CASP-9, } \uparrow \text { Cyt C, } \uparrow \text { MMP-7, } \downarrow \text { p-GSK3 } \beta, \\
\downarrow \beta \text {-catenin }\end{array}$ & $\begin{array}{l}\uparrow \text { Apoptosis, } \downarrow \text { proliferation, } \\
\downarrow \text { lung metastasis, } \downarrow \text { migration, } \downarrow \text { invasion }\end{array}$ & 181 \\
\hline & MDA-MB-231 \& Hs-578T & $\downarrow$ miR-21, $\downarrow$ STAT3 & $\downarrow$ Invasion & 184 \\
\hline & MDA-MB-231 \& Hs-578T & $\uparrow \mathrm{RECK}, \downarrow$ miR-21 & $\downarrow$ Invasion & 185 \\
\hline & $\begin{array}{l}\text { MCF-7 \& MDA-MB-231, } \\
\text { MMTV-PyMT mice }\end{array}$ & $\begin{array}{l}\downarrow \text { DNMT1, } \downarrow \beta \text {-catenin, } \uparrow \text { WIF } 1, \downarrow \text { Wnt, } \\
\downarrow \beta \text {-catenin, } \downarrow \text { Cyclin D1, } \downarrow \text { Survivin, } \\
\downarrow \text { C-Myc, Oct- } 4\end{array}$ & $\begin{array}{l}\downarrow \text { Proliferation, } \downarrow \text { lung metastasis, } \\
\downarrow \text { initiation \& progression, } \\
\downarrow \text { bCSCs \& its self-renewal }\end{array}$ & 187 \\
\hline & BT549 \& MDA-MB-231 & $\begin{array}{l}\downarrow \mathrm{COX}-2, \downarrow \text { CYP } 4 \mathrm{~A}, \downarrow \mathrm{PGE}_{2}, \downarrow \text { PLA2 } \\
\uparrow \text { cleaved caspase-3 \& }-9\end{array}$ & $\begin{array}{l}\downarrow \text { Metastasis, } \uparrow \text { apoptosis, } \\
\uparrow \text { anoikis }\end{array}$ & 333 \\
\hline & BT549, MCF-7 \& & $\downarrow \beta$-catenin, $\downarrow$ ABCG2, $\downarrow$ GRP78, & $\downarrow$ Proliferation, $\downarrow$ colonization, $\uparrow$ apoptosis, & 188 \\
\hline & MDA-MB-231 & $\begin{array}{l}\uparrow \text { proteasome degradation pathway, } \\
\downarrow \text { CD } 44^{+} \text {CD } 24^{-/ \text {low }}, \downarrow \text { Survivin, } \\
\downarrow \text { Cyclin D } 1, \downarrow \text { Oct- } 4, \downarrow \text { c-Myc }, \downarrow \text { GSK- } 3 \beta,\end{array}$ & $\begin{array}{l}\downarrow \text { bCSCs self- } \\
\text { renewal \& differentiation, } \\
\uparrow \text { chemosensitization }\end{array}$ & \\
\hline & $\begin{array}{l}\text { MCF-7, MCF-7/ADR \& } \\
\text { MCF-10A }\end{array}$ & $\downarrow \mathrm{miR}-25, \downarrow$ ABCG2, $\uparrow$ ULK1,$\uparrow \mathrm{LC} 3-\mathrm{II}$ & $\begin{array}{l}\downarrow \text { proliferation, } \uparrow \text { chemosensitization, } \\
\downarrow \text { colonization }\end{array}$ & 186 \\
\hline & MDA-MB-231 & $\begin{array}{l}\downarrow \text { VEGF }, \downarrow \text { HIF- } 1 \alpha, \downarrow \text { MMP- } 2, \downarrow \text { MMP-9, } \\
\downarrow \text { p38, } \text { Akt }, \downarrow \text { NF- } \kappa B, \downarrow \text { P13K }\end{array}$ & $\downarrow$ Migration, $\downarrow$ proliferation, $\downarrow$ angiogenesis & 48 \\
\hline \multirow[t]{3}{*}{$\begin{array}{l}3,3^{\prime}- \\
\text { Diindolylmethane }\end{array}$} & MCF-7 & $\begin{array}{l}\downarrow \text { EMT, } \downarrow \text { CXCR } 4, \downarrow \text { N-cadherin, } \\
\uparrow \text { E-cadherin, } \downarrow \text { snail, } \downarrow \text { slug, } \downarrow \text { cathepsin B, } \\
\downarrow \text { cathepsin D } \downarrow \text { MMP-2, } \downarrow \text { MMP-9 }\end{array}$ & $\downarrow$ Metastasis, $\downarrow$ proliferation & 264 \\
\hline & MCF-7 \& T47D & $\uparrow \mathrm{p} 21$ & $\downarrow$ Proliferation & 334 \\
\hline & MDA-MB-231 & $\downarrow$ Akt & $\downarrow$ Proliferation & 266 \\
\hline
\end{tabular}


Table 2 (Contd.)

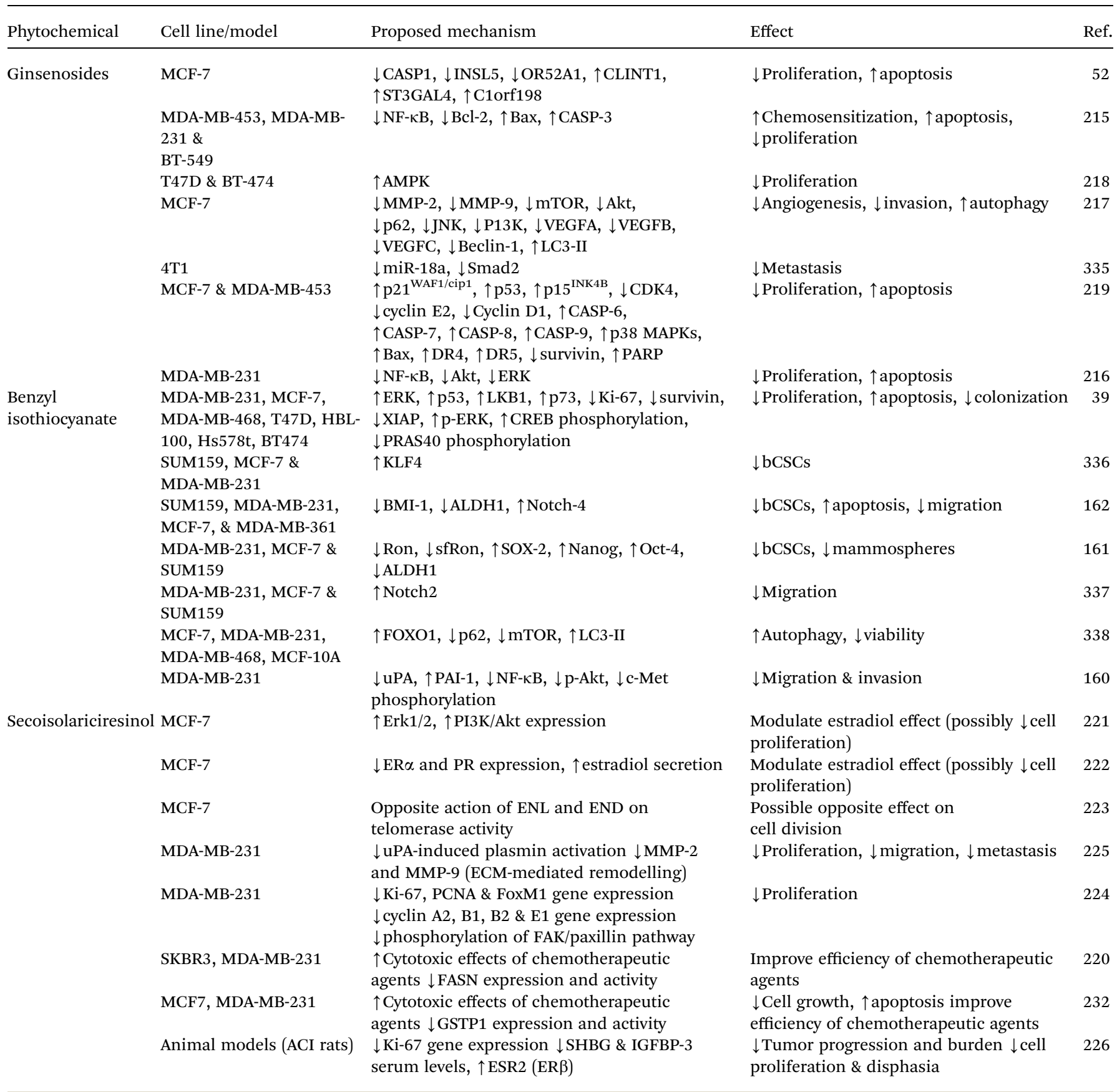

TIMP1/4 genes and inhibiting MMP-2 and MMP-9 in breast cancer cells. ${ }^{86}$ Mo et al. (2012) revealed that curcumin inhibit invasion of MDA-MB-231 cells via reduction of p-ERK and Smad2 signalling. ${ }^{87}$ The compound also affected genes related to Epithelial-Mesenchymal Transition (EMT), leading to reduced expression of $\beta$-catenin, N-cadherin, E-cadherin, Twist1, slug, AXL, fibronectin and vimentin, subsequently inhibiting migration and invasion in breast cancer cells. ${ }^{\mathbf{8 8}}$ Other pathways and genes modulated by curcumin are presented in Table 2 .

\subsection{Epigallocatechin gallate (EGCG)}

EGCG - a polyphenol and predominant catechin in green tea (Camellia sinensis) - has long been studied for its health benefits, including cancer chemoprevention. Sinha et al. (2017) have recently reviewed the role of green tea and its constituents in breast cancer chemoprevention. EGCG induced antiproliferative, anti-metastatic, apoptotic, anti-angiogenic, antigenotoxic and epigenetic effects by targeting several pathways and genes. ${ }^{36}$ There is also evidence that topical EGCG prevented radiation-induced dermatitis in breast cancer patients, thus making it a radio-protective agent. ${ }^{89-91}$ 
EGCG induced apoptosis through downregulation of telomerase and $\mathrm{P} 13 \mathrm{~K} / \mathrm{AKT}$ and increased $\mathrm{Bax} / \mathrm{Bcl}-2$ ratio and $\mathrm{p} 53$ expression in T47D cells. The expression of hTERT gene was decreased while that of CASP3, CASP9 and PTEN was increased..$^{92}$ Huang et al. (2017) also observed an increase in p53 and decrease in Bcl-2 in MCF-7 cells. ${ }^{93}$ Moreover, EGCG suppressed the growth of MDA-MB-231 cells through downregulation of $\beta$-catenin, cyclin D1 and pAkt. ${ }^{94}$ In tumor microenvironment, tumor-associated macrophages (TAM) carry a significant role. EGCG reduced TAM infiltration and M2 macrophage polarization by upregulating miR-16, thereby decreased tumor growth in murine breast cancer model. ${ }^{95}$

EGCG suppressed heat shock protein 90 (Hsp90) and promoted the translocation of progesterone receptor B (PR-B) into the nucleus, consequently resulting in downregulation of ER $\alpha .{ }^{96}$ Physiological concentrations of EGCG inhibited the growth of ER $\alpha$-positive MCF-7 cells through reduction in ER $\alpha$ and insulin-like growth factor (IGF) binding protein 2 and increased p53 and p21 (tumour suppressors). ${ }^{97}$ Furthermore, the compound downregulated expression of ER- $\alpha 36$ and thus suppressed the growth of ER-negative breast cancer stem/ progenitor cells. ${ }^{98}$

EGCG sensitized MCF-7 cells to 5-fluorouracil by regulating the expression of Bcl-xL. ${ }^{99}$ The compound also achieved sensitization to tamoxifen by reducing the expression of Nrf2 (transcription factor) in tamoxifen-resistant MCF-7 cells. ${ }^{\mathbf{1 0 0}}$ Similarly, a reduction in MMP-2 and MMP-9 in doxorubicin resistant MCF-7 cells has also been observed. ${ }^{101}$

\subsection{Genistein}

Genistein - a phytoestrogen in soybeans-primarily exerts its function by interacting with estrogen receptors $(\mathrm{ER} \alpha \& \mathrm{ER} \beta) .{ }^{\mathbf{1 0 2}}$

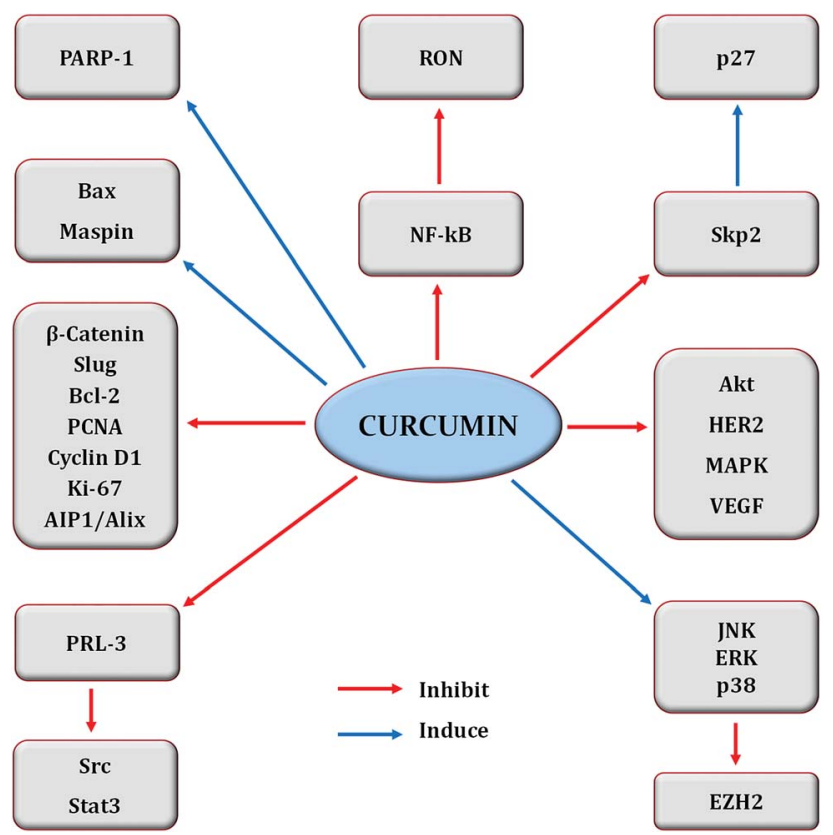

Fig. 6 Regulation of molecular targets by curcumin in breast cancer cells. Idea adopted from Liu and Chen (2013). ${ }^{396}$

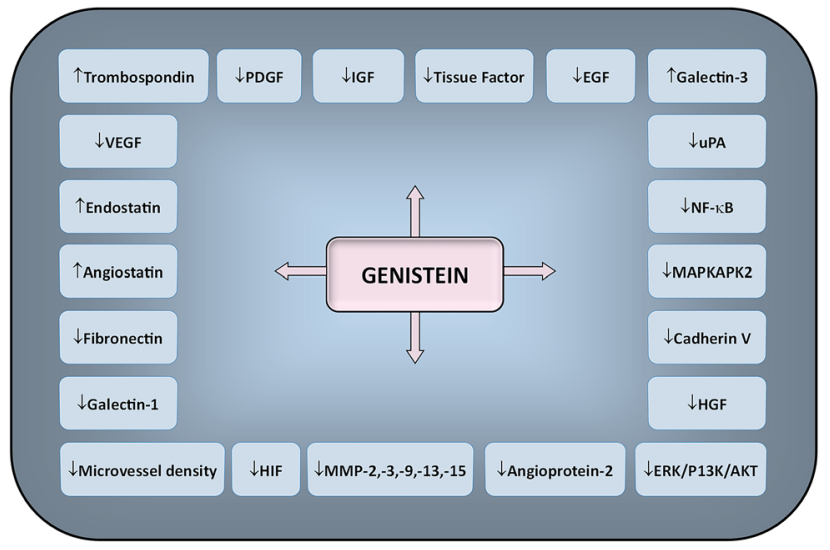

Fig. 7 Molecular targets regulated by genistein in cancer cells. Downward arrows $(\downarrow)$ represent decrease of activity/expression/ secretion while upward arrows $(\uparrow)$ represent increase of activity/ expression/secretion. Idea adopted from Varinska et al. (2015). ${ }^{397}$

Varinska et al. (2015) reviewed the molecular targets regulated by genistein in cancer cells, with special preference to angiogenesis (Fig. 7). ${ }^{397}$ Genistein repressed the differentiation and proliferation of 3T3-L1 and MCF-7 cells by regulating the expression of $E R \alpha .^{103}$ It also reactivated $E R \alpha$ expression by remodelling its promoter chromatin structure and inhibited the growth of ER $\alpha$-negative breast cancer cells. This epigenetic restoration of $\mathrm{ER} \alpha$ further enhanced the therapeutic efficacy of tamoxifen. ${ }^{104}$ Additionally, the compound reduced the expression of ER $\alpha$ and c-erB-2, consequently inhibiting the proliferation of ER $\alpha /$ c-erB-2-positive breast cancer cells. ${ }^{105}$ Genistein also induced apoptosis in MCF-7 and T47D cells via downregulation of CIP2A (cancerous inhibitor of PP2A). ${ }^{106}$ A phosphoproteomic level study using TNBC cells revealed that several biological processes are regulated by genistein during cell cycle such as kinetochore formation, cohesion complex cleavage and DNA replication. Moreover, DNA damage response involving BRCA1 complex and ATR can also be activated by genistein. ${ }^{\mathbf{1 0 7}}$

Genistein resulted in reactivation of several tumor suppressor genes such as adenomatous polyposis coli (APC), mammary serpin peptidase inhibitor (SERPINB5), ataxia telangiectasia mutated (ATM) and PTEN in breast cancer cell lines by decreasing their methylation status. This was accomplished through reduced expression of DNMT1 by genistein. ${ }^{108}$ Moreover, it also suppressed cyclin D1 and DNMT-1 in MCF-7 cells, leading to BRCA1 $\mathrm{CpG}$ demethylation and consequent reactivation of BRCA1. ${ }^{40}$ Prepubertal exposure to Bisphenol A (BPA) changes the signalling pathways which may add to carcinogenesis, as revealed by DNA methylation studies. Conversely, prepubertal exposure to genistein or genistein + BPA resulted in hypomethylation of several genes in rat mammary tissues out of which HPSE and RPS9 genes were linked with enhanced long term survival, thus emphasizing cancer preventive properties of genistein. ${ }^{109}$ In another study, lifetime genistein intake improved response of mammary tumors to tamoxifen resistance in Sprague-Dawley rats and also reduced the risk of recurrence. Genistein administration downregulated the 


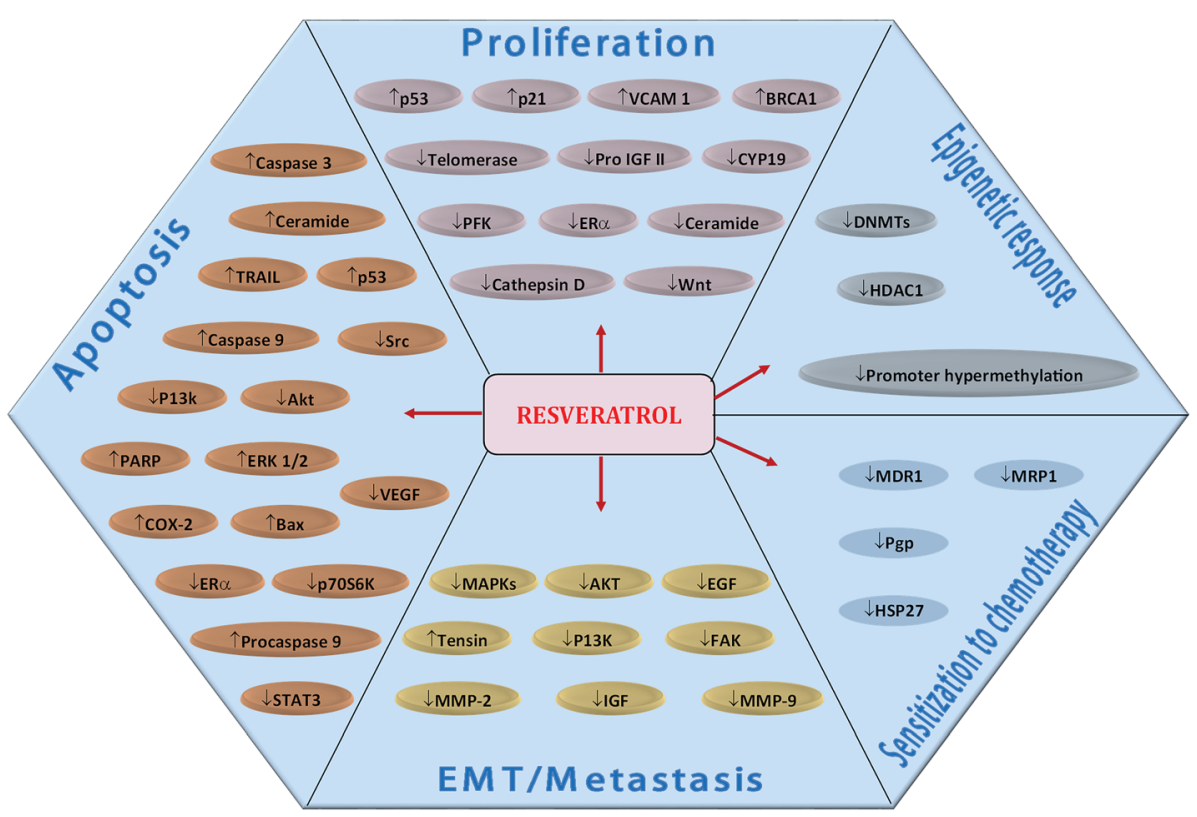

Fig. 8 Resveratrol-mediated regulation of molecular targets underlying breast cancer cells proliferation, apoptosis, EMT/metastasis, epigenetic responses and sensitization to chemotherapy. Downward arrows $(\downarrow)$ represent downregulation while upward arrows $(\uparrow)$ represent upregulation. Idea adopted from Sinha et al. (2016). ${ }^{398}$

unfolded protein response (UPR) and genes linked to autophagy such as ATF4, GRP78, Beclin-1 and IRE1 $\alpha$, as well as, immunosuppression-linked genes like Foxp3 and TGF $\beta$ and upregulated CD8a (cytotoxic T-cell marker) in tumors. This demonstrates that the enhanced response to endocrine therapy was pre-programmed early in life. ${ }^{110}$

Avci et al. (2015) found that genistein suppress the growth of MCF-7 cells via upregulation of miR-23b. ${ }^{111}$ Furthermore, it inhibited NF- $\mathrm{KB}$ via Notch-1 signalling and reduced the growth of TNBC cells. ${ }^{111}$ Long-term low-dose genistein administration sensitized inflammatory breast cancer cell lines to radiation and reduced mammosphere formation and the growth of stem cell populations. ${ }^{112}$ Downregulation of Hedgehog-Gli1 signalling by genistein is also linked with reduced bCSCs. ${ }^{113}$

\subsection{Resveratrol}

Resveratrol (RSV)—a phytoestrogen mainly produced by grapes, berries and peanuts in response to stress conditions-has also been explored well for its anti-malignant properties. Sinha et al. (2016) comprehensively reviewed the effect of RSV on breast cancer cells. ${ }^{398}$ RSV regulate various molecular targets underlying breast cancer cells proliferation, apoptosis, EMT/ metastasis, as well as, epigenetic responses and sensitization to chemotherapy (Fig. 8). ${ }^{398}$ Recently, RSV was found to induce the expression of ATPase sarcoplasmic/endoplasmic reticulum $\mathrm{Ca}^{2+}$ transporting 3 (ATP2A3)-gene encoding enzyme which maintains homeostasis of intracellular $\mathrm{Ca}^{2+}$ by pumping it into endoplasmic reticulum (ER) - in breast cancer cells, thereby induced apoptosis, reduced cell viability and caused alteration in cytosolic levels of $\mathrm{Ca}^{2+}$ along with its releasing capacity by ER. ${ }^{114}$ RSV induced apoptosis in human epidermal growth factor receptor 2 (HER2)-positive SKBR-3 cells via suppression of fatty acid synthase and HER2 genes. The compound also upregulated PTEN expression and down-regulated Akt phosphorylation (pAkt), thereby alleviated P13K/Akt/mTOR pathway. ${ }^{115}$ RSV prevented breast cancer cell invasion by directly inactivating RhoA which in turn phosphorylated YAP via Lats1 activation, leading to reduced expression of YAP gene. ${ }^{116}$ Moreover, it downregulated the polo-like kinase-1 and aurora kinase A (cell cycle regulatory proteins), cyclin D1 and cyclin B1, thereby suppressed cell cycle in breast cancer cells. ${ }^{117}$

Combination of RSV with other compounds greatly improved its efficacy and bioavailability. Co-treatment of thymoquinone and RSV increased apoptosis, induced geographic necrosis, reduced VEGF and increased serum levels of IFN- $\gamma$ in breast cancer cell lines. ${ }^{118}$ Likewise, combination with salinomycin markedly enhanced the anti-cancer properties of RSV in breast cancer cells via downregulation of canonical Wnt signalling proteins and vimentin. ${ }^{119}$ Similarly, RSV and pterostilbene reactivated the expression of $\mathrm{ER} \alpha$ in $\mathrm{ER} \alpha$-negative breast cancer cell lines by increasing active chromatin markers such as acetyl-H3lysine 9 and acetyl-H3/4 in ER $\alpha$ promoter region. Reduction in the activity of DNMT and level of 5-methylcytosine along with alteration in histone acetyl transferase and HDAC were also noted. ${ }^{120}$ Enhanced apoptosis of MCF-7 cells occurred when Sorafenib (drug inhibitor of angiogenesis and tyrosine kinase) was used in combination with RSV. The treatment induced the expression of p53, enhanced intracellular ROS generation and Bax/Bcl-2 expressions while reduced mitochondrial membrane potential. Moreover, downregulation of cyclin B1 and cyclin D1 also occurred while cleaved caspase-3, -9 , cleaved poly(ADP-ribose) polymerase (PARP) and apaf- 1 were found to be upregulated. ${ }^{121} \mathrm{RSV}$ and rapamycin prevented mTORC1 (mechanistic target of rapamycin), autophagy and Akt 
activation, consequently instigating apoptosis in breast cancer cells. ${ }^{122}$ Co-treatment with melatonin inhibited aromatase, thus making them suitable for hormonal treatment of breast cancer. ${ }^{123}$ RSV also sensitized resistant MCF-7/DOX cells to doxorubicin via downregulation of MDR1. ${ }^{124}$ Furthermore, the compound enhanced the cellular accumulation of doxorubicin by reducing $\mathrm{ABC}$ transporter genes, MRP1 and MDR1 in resistant breast cancer cells. ${ }^{54}$ In another study, down-regulation of HSP27 by RSV has been linked with sensitization to doxorubicin in resistant MCF-7 cells. ${ }^{125}$ Similarly, RSV chemosensitized resistant MCF-7 cells to tamoxifen by modulating Smad phosphorylation and endogenous TGF- $\beta$ production, ${ }^{\mathbf{1 2 6}}$ and to melphalan by arresting cell cycle at $S$ phase. ${ }^{127}$

Resveratrol modulation of microRNAs has also been observed. Venkatadri et al. (2016) demonstrated that the compound modulate crucial tumor-suppressive microRNAs such as miR-122-5p, miR-125b-5p, miR-409-3p, miR-542-3p and miR-200c-3p in breast cancer cells. These microRNAs regulate key cell cycle and anti-apoptotic proteins like X-linked inhibitor of apoptosis protein, Bcl-2 and CDKs. ${ }^{128}$

\subsection{Quercetin}

Quercetin-a well-researched and potential chemopreventive agent ubiquitous in plant foods-suppressed the growth of T47D cells by inhibiting the gene expression and secretion of leptin, thus making leptin a novel target in breast cancer therapy. ${ }^{129}$ The compound increased Bax and decreased Bcl-2 proteins expressions in MCF-7 cells. In the presence of Nec-1 (necroptosis inhibitor), the expression of Bax and apoptotic index decreased and an increase in proliferation and viability of MCF-7 cells occurred, suggesting that the death pathway mainly involve necroptosis. ${ }^{\mathbf{1 3 0}}$ Additionally, quercetin induced apoptosis in TNBC cells by reducing the expressions of $\beta$-catenin and fatty acid synthase (FASN). ${ }^{44}$ Similarly, the compound displayed apoptotic activity in MCF-7 cells by inhibiting Twist through p38MAPK pathway. ${ }^{\mathbf{1 3 1}}$ Likewise, anti-proliferative and apoptotic activity in MCF-7 cells via reduced survivin expression and Go/G1-phase arrest was also reported. ${ }^{132}$

Combination therapy of quercetin and ascorbic acid (vitamin C) along with therapeutic drugs such as paclitaxel or doxorubicin markedly enhanced their anti-cancer properties in breast cancer cells by significant reduction in $\mathrm{S}$ and Go/G1 phases. ${ }^{133}$ Quercetin downregulated Her-2 and upregulated ER $\alpha$, thereby reversed tamoxifen resistance in MCF-7 cells. ${ }^{\mathbf{1 3 4}}$ Pentagalloylglucose (5GG), which shows structural resemblance to (-)-EGCG, in combination with quercetin persuaded S-phase arrest and caused apoptosis in MDA-MB-231 cells via decreased expression of S-phase kinase, while G2/M-phase arrest and apoptosis in AU565 cells occurred through reduced expression of Her-2. ${ }^{135}$

Quercetin revealed anti-metastatic property in TNBC cells by modulating EMT markers. It increased E-cadherin and decreased vimentin, leading to mesenchymal-to-epithelial transition, associated with modulation of cyclin D1 and c-Myc ( $\beta$-catenin target genes) and a change in $\beta$-catenin nuclear localization. ${ }^{136}$ The calcineurin/NFAT pathway play a key role in angiogenesis which was inhibited by quercetin in breast cancer xenograft via downregulation of VEGF, VEGFR2 and NFATc3. ${ }^{137}$ Quercetin up-regulated miR-146a and prevented proliferation in breast cancer cells. The compound also inhibited invasion via EGFR suppression and induced mitochondrial-mediated apoptosis. ${ }^{\mathbf{1 3 8}}$

\subsection{Silibinin}

Silibinin-a main active constituent of silymarin complex extracted from Silybum marianum-has a potent hepatoprotective activity. ${ }^{139}$ The compound also displayed anticancer properties and prevented breast cancer cells metastasis through inhibition of CXCR4 (chemokine receptor type 4). ${ }^{\mathbf{1 4 0}}$ Moreover, it suppressed the migration of MCF-7 cells and markedly reduced MMP-9 expression via inhibition of p-ERK and p-MEK. $^{\mathbf{1 4 1}}$ It also inhibited proliferation and induced apoptosis in ER $\alpha$-negative SKBR3 cells via suppression of NF- $\kappa$ B. ${ }^{142}$ Silibinin decreased p53, p21, Bcl-xL, Bak and BRCA1 in MCF-7 cells, thereby induced apoptosis and prevented proliferation. ${ }^{143}$ Furthermore, it conveyed anti-tumorigenic property in TNBC xenograft model by inhibiting the phosphorylation of EGFR and subsequently suppressing VEGF, MMP-9 and COX-2. ${ }^{144}$

Co-treatment of silibinin with cisplatin or paclitaxel increased early apoptosis by decreasing Bcl-2 and increasing mRNA levels of Bax, ATM, BRCA1 and p53 in MCF-7 cells. ${ }^{145}$ Silibinin also sensitized resistant breast cancer cells to paclitaxel and doxorubicin through inhibition of the key oncogenic pathways encompassing ERK, AKT and STAT3 in doxorubicinresistant MDA-MB-435 cells and paclitaxel-resistant MCF-7 cells at $400 \mu \mathrm{M}$ concentration. ${ }^{\mathbf{1 4 6}}$ Similarly, silibinin and chrysin synergistically inhibited proliferation of T47D cells via downregulation of cyclin D1 and hTERT. ${ }^{147}$

Silibinin reduced proliferation of MCF-7 cells via downregulation of miR-155 and miR-21 and induced apoptosis through intrinsic and extrinsic pathways by upregulating their apoptotic targets such as BID and CASP-9. ${ }^{\mathbf{1 4 8}}$ However, Jahanafrooz et al. (2017) presented that down-regulation of miR-21 upon silibinin treatment has minimal effect on its antitumorigenic property in breast cancer cells and that other pathways are responsible for its anti-apoptotic affect. ${ }^{\mathbf{1 4 9}}$

Silibinin exerted autophagic cell death in MCF-7 cells via ROS-dependent mitochondrial dysfunction $(\Delta \Psi \mathrm{m})$ and a reduction in ATP levels involving BNIP3 (Bcl-2 interacting protein 3, a member of pro-death Bcl-2 protein family). ${ }^{\mathbf{1 5 0}}$ However, according to Zheng et al. (2015), the anti-apoptotic and autophagy induction properties of silibinin in ER $\alpha$-positive MCF-7 cells are due to down-regulation of ER $\alpha$ expression and subsequent inhibition of ERK and mTOR signalling pathways. ${ }^{151}$

\subsection{Thymoquinone}

Thymoquinone (TQ) — an anti-inflammatory, antioxidant, anticancerous and cytotoxic constituent in black seed oil-has also been investigated in breast cancer therapy. The compound led to differential expression of apoptosis related genes in ER- 
positive (MCF-7) cells. Up-regulation of PTPRR gene resulted in inhibition of p38-MAPK and MAPK pathways. Inhibition of p38MAPK triggered down-regulation of Bcl-2 and up-regulation of TP53 which indicates that intrinsic apoptotic pathway is involved. Moreover, participation of caspase in apoptosis was confirmed through downregulation of CARD16 gene. ${ }^{152}$ Recently, the effect of TQ on miRNA profile and molecular mechanism using MCF-7 cells was highlighted and it was found that hsa04310 (Wnt), hsa04115 (p53), hsa04151 (P13K/AKT) and hsa04010 (MAPK) are the key targets of the compound. ${ }^{153}$ TQ pre-sensitization restored cytokeratin 19 and E-cadherin (epithelial markers), as well as, MMP-2, MMP-9, integrin- $\alpha \mathrm{V}$ (mesenchymal markers) and TGF- $\beta$ in radiation-induced metastatic progression of breast cancer. ${ }^{154}$ TQ also exerted anticancer action in breast cancer cells by attenuating the activity of global histone deacetylase (HDAC). The compound increased Bax, decreased Bcl-2, reactivated HDAC target genes (maspin and p21) and induced G2/M phase arrest. ${ }^{155}$

TQ and tamoxifen synergistically induced apoptosis and decreased cell viability in both estrogen positive (MCF-7) and estrogen negative (MDA-MB-231) cell lines. ${ }^{156}$ The compound sensitized breast cancer cells to paclitaxel through multiple cascades involving extrinsic apoptosis, p53 signalling and tumor suppressor genes. Moreover, TQ regulated apoptosis inducible genes through death receptors and upregulated tumor suppressor genes including BRCA1, p21 and Hic1. Additionally, high doses of TQ downregulated pro-apoptotic factors like caspases and upregulated growth factors such as EGF and VEGF. ${ }^{157}$ Likewise, co-treatment of thymoquinone and melatonin greatly decreased tumor size via induction of apoptosis, activation of Th1 immune response and inhibition of angiogenesis. ${ }^{158}$

\subsection{Benzyl isothiocyanate}

Benzyl isothiocyanate (BITC) - an isothiocyanate from cruciferous vegetables-exhibit anti-carcinogenic effect. BITC induced apoptosis in breast cancer cells by altering mitochondrial dynamics. The compound greatly reduced levels of Drp1, p-S616, Fis1 (fission proteins) and Mfn1, Mf2 (fusion proteins) in breast cancer cells, suggesting that it negatively target the fission and fusion machinery of mitochondrial dynamics. Furthermore, it was shown that multi-domain pro-apoptotic proteins Bak and Bax regulated the BITC-mediated inhibition of mitochondrial fusion. ${ }^{159}$ BITC triggered p53-signalling network and repressed p53-mutant cells growth. The compound induced expression of p73 in these mutant cells, interrupting the interaction of mutant-p53 and p73 and subsequently emancipating p73 from sequestration and permitting it to be active transcriptionally. In molecular mechanisms, liver kinase B1 (LKB1), a tumour suppressor, was found to be a key node underlying the anti-cancer role of the compound. The p73 and p53 transcriptionally upregulated LKB1 in a mutant- and wild-type-p53 breast cancer cells, respectively. Furthermore, it was elucidated that LKB1 tethers with p73 and p53 in a feed-forward mechanism for recruitment into p53-responsive gene promoters. ${ }^{39}$ BITC also suppressed the migration and invasion of MDA-MB-231 cells through decreased uPA activity and reduction of p-Akt. ${ }^{\mathbf{1 6 0}}$

Truncated Recepteur d'Origine Nantais (sfRON) was also found as a novel mechanistic target for induction of apoptosis in breast cancer cells. BITC induced apoptosis in MDA-MB-361 and MCF-7 cells by regulating sfRON, whereby overexpression of sfRON in these cells intensified apoptosis, independent of JNK or MAPK hyperphosphorylation. Moreover, activation of Bak and Bax in sfRON overexpressing cells following BITC treatment were greatly enhanced while G2/M phase arrest and ROS generation were attenuated. ${ }^{159}$ However, according to Kim et al. (2013), full-length RON and sfRON overexpression offered protection against inhibition of bCSCs by BITC in MCF-7 cells and down-regulation of RON and its truncated form are associated with breast cancer stem cells inhibition by BITC. ${ }^{161}$ BITC inhibited bCSCs via downregulation of oncogene BMI-1 (Polycomb complex protein) and activation of Notch-4 in in vivo and in vitro breast cancer cells. ${ }^{162}$

\subsection{Apigenin}

Apigenin-a well-known anti-inflammatory flavonoid in parsley and variety of other plants-suppressed TNF $\alpha$-mediated release of chemokines including CCL2, IL-1 $\alpha$, IL-6, and granulocyte macrophage colony-stimulating factor in TNBC cells. These chemokines direct the inward migration/infiltration of tumorassociated neutrophils, T-regulatory cells, tumor-associated macrophages, T-helper IL-17-producing cells, myeloid-derived suppressor cells, cancer-associated fibroblasts and metastasisassociated macrophages, which collectively enables tumor growth, immune evasion, metastasis and angiogenesis. The inhibition of CCL2 by apigenin occurred through suppression of IKBKe signalling. ${ }^{\text {163-172 }}$ Apigenin overcame drug resistance and decreased colonization and cell growth in adriamycinresistant MCF-7 cells via downregulation of MDR1 and Pglycoprotein, as well as, suppression of STAT3 signalling and its nuclear translocation. The compound also diminished the secretion of MMP-9 and VEGF (STAT3 target genes) in these cells. ${ }^{173}$

Moreover, apigenin inhibited the proliferation and clonogenic survival of HER2-expressing BT-474 cells via caspasedependent extrinsic apoptosis by upregulating cleaved caspase-3, cleaved caspase- 8 and PARP cleavage. The compound decreased phospho-STAT3, phospho-JAK1 and phospho-JAK2 and prevented $\mathrm{CoCl}_{2}$-induced VEGF secretion and STAT3 nuclear translocation in HER2-expressing breast cancer cells. ${ }^{174}$ The same authors also mentioned before that apigenin induce apoptosis in HER2-overexpressing MCF-7 cells via extrinsic pathway, inhibit NF-кB and STAT3 and induce p53. ${ }^{175}$

Mechanism behind induction of cell cycle arrest by apigenin in MDA-MB-231 cells was also pointed out and it was shown that the compound suppress cyclin dependent kinase-1 (CDK1), cyclin A and cyclin B which are vital for G2-to-M-phase transition in cell cycle. Furthermore, apigenin increased p21 ${ }^{\text {WAF1/CIP1 }}$ and its interaction with proliferating cell nuclear antigen, thus obstructing cell cycle progression. Moreover, inhibition of histone deacetylase activity and an increase in acetylated 
histone H3 was also observed. ${ }^{176}$ Harrison et al. (2014) reported that apigenin reduce the proliferation of MDA-MB-468 cells by arresting cell cycle at $\mathrm{G} 2 / \mathrm{M}$ phase and enhancing ROS production. Additionally, the compound reduced p-Akt. ${ }^{177}$

\subsection{Isoliquiritigenin}

Isoliquiritigenin (ISL) - a flavonoid mostly found in the root of liquorice-exhibits anti-cancer properties at multistage carcinogenesis processes such as cell cycle arrest, proliferation suppression, apoptosis induction, metastasis obstruction and angiogenesis inhibition in different kinds of cancer. ${ }^{178-183}$

Peng et al. (2017) proposed that ISL induces apoptosis and prevent metastasis in breast cancer cells via downregulation of miR-374a. Decreased expression of miR-374a led to increased PTEN expression which prevented abnormal Akt signalling. ${ }^{\mathbf{1 8 1}}$ ISL transcriptionally downregulated the expression of primary and mature miR-21 and decreased STAT3 signalling activity in breast cancer cells. ${ }^{184}$ The compound also suppressed breast cancer cells invasion by upregulating RECK (tumor suppressor gene) and downregulating miR-21. ${ }^{185}$ Moreover, ISL caused cell cycle arrest, chemosensitized and induced autophagy in MCF-7/ ADR cells and also stimulated the degradation of ABCG2 through autophagy-lysosome pathway. Autophagy induction was associated with inhibition of miR-25 which led to increased expression of ULK1 (a kinase involved in autophagy). ${ }^{186}$ ISL treatment inhibited NF- $\mathrm{B}, \mathrm{P} 13 \mathrm{~K} / \mathrm{Akt}$ and p38, ensuing decreased expressions of MMP-2, MMP-9, VEGF and HIF-1 $\alpha$ which reduced the migration of breast cancer cells. ${ }^{48}$

ISL encouraged the demethylation of WIF1 promoter by docking into the catalytic domain of DNMT1, thereby increasing the gene expression of WIF1 and subsequently prevented mammary carcinogenesis by inhibiting bCSCs. ${ }^{187}$ The compound also chemosensitized bCSCs by inhibiting $\beta$-catenin/ABCG2 signalling via docking into ATP domain of GRP78 (binding immunoglobulin protein), resulting in inhibition of its ATPase activity and subsequent dissociation from $\beta$-catenin. ${ }^{188}$

\subsection{Pterostilbene}

Pterostilbene-resveratrol analogue extracted from blueberries-markedly inhibited the growth and induced apoptosis in TNBC cells through G0/G1 phase arrest. The compound maintained the activation of ERK1/2, associated with decreased cyclin D1 and increased p21 expressions. Moreover, it also repressed p-AKT and mTOR and a subsequently increased Bax protein without affecting Bcl-xL. ${ }^{189}$ Pterostilbene enhanced TNFrelated apoptosis-inducing ligand (TRAIL)-induced apoptosis in TNBC cells via ROS-mediated activation of p38/C/EBPhomologous protein pathways which subsequently induced the expression of death receptors DR4 and DR5. The compound also reduced the expression of decoy receptor 1 and decoy receptor 2 and suppressed the anti-apoptotic proteins Bcl-2, Bcl$\mathrm{xL}$, XIAP, c-FLIPS/L and survivin, as well as, increased Bax and Bid cleavage in these cells. ${ }^{\mathbf{1 9 0}}$

Pterostilbene inhibited metastasis of TNBC cells through induction of miR-205 and negative modulation of EMT markers including vimentin, ZEB1, slug and snail and upregulation of E- cadherin. Downregulation of miR-205 caused decreased expression of Src-a nonreceptor tyrosine kinase whose activation and overexpression leads to different types of cancers, involving invasive breast cancer. ${ }^{191-194}$

Interestingly, pterostilbene and 6-shogaol inhibited bCSCs via reduced expression of $\mathrm{CD} 44^{+}$surface antigen and stimulated phosphorylation of $\beta$-catenin through hedgehog/Akt/GSK3 $\beta$ signalling inhibition thus downregulating downstream cyclin D1 and c-Myc. ${ }^{195}$ The occurrence of M2-polarized tumour associated macrophages (TAMs) enhanced metastatic abilities and $\mathrm{CD}_{4} 4^{+} / \mathrm{CD}^{-}$CSCs in breast cancer cells. M2 TAMs-associated properties were suppressed by pterostilbene via inhibition of NF- $\kappa \mathrm{B}$, E-cadherin, vimentin and Twist1. ${ }^{196}$

\subsection{Sulforaphane}

Sulforaphane (SFN) - an antioxidative and anti-inflammatory compound found in cruciferous vegetables-modulates various cellular targets that are involved in cancer development. ${ }^{197-203}$ SFN stimulated the anti-cancer activity of taxane (docetaxel or paclitaxil) in TNBC cells by preventing CSCs via reduced translocation of NF- $\kappa \mathrm{B}$ p65 and decreased p52 expression. The compound also restored taxane-induced ALDH+ cell enrichment and markedly suppressed mammospheres. ${ }^{\mathbf{2 0 4}}$ Additionally, co-treatment of SFN and paclitaxel enhanced paclitaxel-induced apoptosis in breast cancer cells through upregulation of cytochrome c, caspase-3, -8 and -9 and downregulation of $\mathrm{NF}-\kappa \mathrm{B}, \mathrm{Bcl}-2$ and $\mathrm{p}$-Akt serine/threonine kinase. ${ }^{205}$ SFN prevented TNF- $\alpha$ mediated migration and invasion in breast cancer cells and suppressed MMP-2, -9 and -13 at both transcriptional and translational levels. ${ }^{206}$ Lee et al. (2012) reported before that SFN suppress MMP-9 expression and invasion in MCF-7 cells via inhibition of NF- $\kappa \mathrm{B}{ }^{207}$

Atwell et al. (2015) reviewed that most of the chemopreventive actions of SFN in breast and prostate cancers are due to modification of epigenetic mechanisms. ${ }^{208}$ Co-treatment of sulforaphane and withaferin A synergistically induced apoptosis and reduced cell viability and epigenetic processes in breast cancer cell lines through upregulation of Bax and downregulation of histone deacetylase 1 (HDAC1) and Bcl-2.. ${ }^{209}$ On the other hand, SFN-induced senescence and cell cycle arrest in breast cancer cells are arbitrated by epigenetic alterations including decreased DNMT1 and DNMT3B expressions, global DNA hypo-methylation and variations in microRNA profile. The compound reduced methylation of $\mathrm{N}_{6}$-methyladenosine RNA (epigenetic regulation at RNA level) and exerted cytostatic action via induction of nitro-oxidative stress and downregulation of Akt signalling. ${ }^{210}$

\subsection{Ginsenosides}

Ginsenosides-major pharmacologically active saponins in ginseng root-are well-known for their promising restorative and healing abilities. Ginsenosides include Rg3, Rb1, Rb2, Rh2, Rh3 (protopanaxadiols) and Rh1, Rg1, Rg2 (protopanaxatriols). ${ }^{211-213}$

Ginsenoside Rh2 provoked epigenetic methylation alteration in genes that are involved in immunity and tumorigenesis, thus 
increased immunogenicity and inhibited the growth of MCF-7 cells. Hyper-methylated genes like INSL5, OR52A1 and CASP1 experienced downregulation, while hypo-methylated genes including C1orf198, ST3GAL4 and CLINT1 displayed upregulation. Moreover, LINE1 (a global methylation marker) also displayed hypomethylation at specific CpGs. ${ }^{52} \mathrm{Rh} 2$ also reversed resistance to docetaxel or adriamycin in resistant MCF-7 cells by differential microRNA expressions including miR-34a, miR-222 and miR-29a. ${ }^{214}$

Ginsenoside $\mathrm{Rg} 3$ enhanced cytotoxic effect of paclitaxel in TNBC cells via inhibition of NF- $\kappa \mathrm{B}$ and $\mathrm{Bcl}-2$ and upregulation of Bax and caspase-3. ${ }^{215}$ Kim et al. (2014) also reported that inhibition of NF- $\kappa \mathrm{B}$ via inactivation of Akt and ERK is responsible for Rg3-induced apoptosis in breast cancer cells. ${ }^{\mathbf{2 1 6}} \mathrm{Rg} 3$ combined with recombinant human endostar suppressed the growth of breast cancer, inhibited cell invasion and angiogenesis and increased autophagy via decreased mRNA contents of MMP-2, MMP-9, VEGFA, VEGFB, VEGFC, p62, Beclin-1, P13K, mTOR, Akt and JNK. ${ }^{217}$

Similarly, ginsenoside Rg5 suppressed the proliferation of breast carcinoma via enhanced activation of AMPK and subsequent reduction in S6 and p70S6K activation. ${ }^{218}$ The compound induced apoptosis via regulation of Bax, cytochrome $\mathrm{c}$ and PARP and promoted cell cycle arrest at G0/G1 phase through downregulation of CDK4, cyclin E2 and cyclin D1 and upregulation of p21 ${ }^{\mathrm{WAF} 1 / \mathrm{CIP} 1}, \mathrm{p} 53$ and p15 ${ }^{\mathrm{INK} 4 \mathrm{~B}}$ in breast cancer cell lines. ${ }^{219}$

\subsection{Secoisolariciresinol and derived enterolignans}

Secoisolariciresinol (SECO) is the most widespread phytoestrogenic lignan (diphenolic compound), abundantly found in flaxseed. Following their ingestion, plant phytoestrogenic lignans are converted into enterolignans (aka mammalian lignans): enterodiol (END) and enterolactone (ENL) that can bind to ER and are therefore considered as selective estrogen receptor modulator (SERM). During the last two decades, evidences suggest that lignan consumption and more precisely the serum lignan concentration are linked with a reduction of breast cancer incidence, ${ }^{220}$ but the last half decade was very informative concerning the possible underlying molecular mechanism.

Examination of the estrogenic activity of ENL using DNA microarray based gene expression profiling in MCF-7 cells revealed that both estradiol and ENL initiated the same estrogen signalling but their signals are then differentially and directionally modulated later in the pathway, resulting in the differences at cell function levels. ${ }^{221}$ Particularly, ENL have been shown to activate the ERK1/2 and PI3K/Akt pathways. ${ }^{221}$ ENL have significantly decreased both ER $\alpha$ and PR (progesterone receptor) expression and increased estradiol secretion in MCF-7 cells. Different dose-responses were observed between ENL and END, with END acting at highest concentration level. ${ }^{222}$ In the same way ENL and END produced opposite action on telomerase activity since ENL, but not END, have been shown to decrease telomerase activity in MCF-7 cells. ${ }^{223}$

Antiproliferative action of lignans was also observed in MDAMB-231 breast cancer cells and has been attributed to the gene expression downregulation of Ki-67, PCNA and FoxM1. ${ }^{224}$ It also exerts a control on cell cycle by lowering gene expression of cyclin E1, A2, B1 and B2 and interfering with the cytoskeleton by downregulating phosphorylation of FAK/paxillin pathway, thus suppressing cell migration and invasion. ${ }^{224}$ ENL have been shown to suppress proliferation, migration and metastasis using MDA-MB-231 breast cancer cells through the reduction of uPA-induced plasmin activation and the matrix metalloproteases MMP-2 and MMP-9 mediated by ECM remodelling. ${ }^{225}$

A reduced tumor progression and burden was observed in a model deriving from ACI rats either with flaxseed or with SECO supplemented groups in both xerograph and carcinogen animal models of human breast cancer. ${ }^{226}$ Hypothesized mechanisms include reduction in estrogen related signalling, anti-angiogenic and antioxidant activity at higher doses. Decrease in serum levels of follicular phase SHBG and IGFBP-3 were noted in supplemented animals, whereas, ESR2 (ER $\beta)$ gene expression increased. ${ }^{226}$ Authors suggested that ESR2 can oppose or reverse the proliferation signalling in mammary epithelial tissue. SECO may activate this pathway, suggesting that increased ER $\beta$ signalling could be one mechanism behind the decrease of mammary proliferation and dysplasia observed with SECO administration. Together a decrease in the cell proliferation marker Ki-67 gene expression was also observed for flax and SECO supplemented animals. SECO also did not promote ovarian dysplasia in these models. ${ }^{226}$

High lignan exposure have been associated with reduced mortality in breast cancer patients. For examples recent studies have found high ENL blood concentrations or high dietary lignans intake to be associated with a better prognosis among post-menopausal women. ${ }^{227,228}$ In a population of 2182 breast cancer patients, ENL was found to be associated with reduced all-cause mortality and breast cancer-specific mortality. This association was restricted to early stage breast cancer and to patients with normal BMI. ${ }^{227}$ However, no association between ENL and HER2 status and Ki-67 gene expression were observed $^{229}$ which is in good agreement with a smaller study ( $n$ $=24$ ) that also reported no effect on Ki-67 gene expression together with no impact on both ER $\beta$ and CASPs expressions. ${ }^{230}$ On the contrary, a previously reported by Flower et al. (2005), a meta-analysis of 10 clinical studies evidenced both the antiproliferative and antiangiogenic activities for flax lignans. ${ }^{231}$ Interestingly, these effects appeared to be correlated with BMI, with significant actions restricted to normal BMI $(<25)$, but without any significant correlation with the menopausal or the $\mathrm{ER}+\mathrm{ER}-$ status of the patients. These observational data suggested associations between flax intake and decreased risk of primary breast cancer, lower mortality among breast cancer patients and better mental health. ${ }^{231}$ A large pre-diagnostic plasma ENL levels and breast cancer prognosis among Danish post-menopausal women study evidence interesting results in crude models higher ENL levels were associated with lower risk of breast cancer-specific mortality but after adjustment for lifestyle factors this association was only borderline significant $(P=0.0501)$. However for women who never smoked or used 
hormones a more significant lower risk of mortality was noted and certainly deserve further investigations. ${ }^{229}$

Flaxseed lignan SECO and ENL were also reported to enhance the cytotoxic effect of classic chemotherapeutic agents (docetaxel, doxorubicin and carboplatin) in the metastatic breast cancer cell lines SKBR3 and MDA-MB-231, suggesting possible future direction in improving chemotherapeutic efficacy in breast cancer by adjuvant therapy with flaxseed lignans. ${ }^{220}$ Combination of dietary supplement along with the reduction in dosage of conventional breast cancer chemotherapeutic agent (doxorubicin) may retain its benefits while minimizing the cytotoxic side effects and thus may enhance its therapeutic efficiency. ${ }^{232}$ Interaction of SECO with gluthathione$S$-transferase PI-1 (GSTP1) gene has also been observed. Inhibition of GSTP1 expression at both gene and protein level by SECO was confirmed in MCF-7 and MDA-MB-231 breast cancer cells. SECO was also shown to inhibit cell growth and increase cellular apoptosis by up-regulating pro-apoptotic Casp-3 and Bax expression and down-regulating anti-apoptotic Bcl-xl and Bcl-2 expression. ${ }^{232}$

\subsection{Vinca alkaloids}

Vinca alkaloids, such as vincristine and vinblastine, are antimicrotubule and antimitotic agents which are obtained from Catharanthus roseus. ${ }^{399,400}$ Combination of vincristine and Thymus caramanicus extract significantly induced cell death in MCF-7 breast cancer cells by increasing protein expression of cleaved caspase 3 and decreasing cyclin D1. ${ }^{233}$ Similar synergistic effect of vincristine and Sutureja khuzestanica extract has also been observed for MCF-7 cells. ${ }^{234}$ On the other hand, vincristine combined with suspension culture has been used for the isolation of cancer stem cells from MDA-MB-231 cell lines. ${ }^{235}$ Recently, dasatinib plus vincristine liposomes were successfully employed for the treatment of triple negative breast cancer using MDA-MB-231 cells and its xenografts in nude mice. The liposomes induced apoptosis via activation of caspase-3, -8 and -9 , increased ROS generation and Bax expression while decreasing Mcl-1. Furthermore, deletion of vasculogenic mimicry channels also occurred. ${ }^{236}$

Vinblastine is extensively used for the treatment of breast cancer and Kaposi's sarcoma. ${ }^{\mathbf{2 3 8 , 4 0 1}}$ Vinblastine not only inhibits the tumor growth but also malignant angiogenesis and can specifically bind to tubulin, thereby restricting its polymerization and subsequent microtubules association. ${ }^{237}$ Recently, it was shown that vinblastine induced apoptosis is MCF-7 cells by interfering with microtubules. The compound increased PARP cleavage, however, it was demonstrated that colchicine was more effective than vinblastine in inducing apoptosis in docetaxel resistant breast cancer (MCF- $7_{\text {TXT }}$ ) cells. ${ }^{238}$ Interestingly, it was observed that enhanced sensitization of MDA-MB-231 cells to vinblastine or actinomycin $\mathrm{D}$ can be achieved by disrupting the function of Golgi apparatus inside these cells, thus resulting in increased apoptosis and decreased cell migration and proliferation. ${ }^{239}$ Likewise, co-treatment of cells with vinblastine or actinomycin D and golgicide A or brefeldin A (disrupting agents of ADP-ribosylation factor 1 (ARF1) - a protein required for homeostasis of Golgi complex) caused the similar effects via reducing the levels of either phospho-AKT or phospho-ERK1/ $2 .^{239}$ Furthermore, combined treatment of MDA-MB-231 cells with vinblastine and docetaxel decreased survivin expression and induced apoptosis. A synergistic effect was observed when deguelin (a survivin inhibitor) was used along with vinblastine and docetaxel, suggesting that survivin downregulation may be useful in increasing the therapeutic efficacy of these chemotherapeutics. ${ }^{240}$

\subsection{Miscellaneous phytochemicals}

Kaempferol. A flavonoid antioxidant occurring in various fruits and vegetables-suppressed the migration and invasion of TNBC cells via downregulation of RhoA and Rac1. ${ }^{42}$ Azevedo et al. (2015) reported that kaempferol exert cytotoxic and antiproliferative actions in MCF-7 cells through inhibition of GLUT1-mediated ${ }^{3} \mathrm{H}$-deoxy-D-glucose uptake. ${ }^{241}$ The compound induced apoptosis in MCF-7 cells by increasing PARP cleavage, decreased Bcl-2 and enhanced Bax expression. ${ }^{242}$ Kaempferol reduced triclosan- or $17 \beta$-estradiol-induced cell growth in MCF-7 cells by downregulating protein expressions of capthepsin D, cyclin E and cyclin D1 and upregulating Bax and p21. Additionally, it reversed triclosan-induced phosphorylation of AKT, MEK1/2 and IRS-1. ${ }^{243}$ Likewise, kaempferol also attenuated triclosan-induced EMT markers via upregulation of E-cadherin (epithelial marker) and downregulation of snail, slug, $\mathrm{N}$ cadherin (mesenchymal markers), along with reduced metastatic markers like MMP-2 and -9, and cathepsin B and -D in MCF-7 cells. ${ }^{244}$

$\boldsymbol{\alpha}$-Mangostin. A xanthone from mangosteen pericarpprompted apoptosis and cell cycle arrest in various human malignancies. ${ }^{\mathbf{2 4 5 - 2 5 0}}$ It persuaded anti-tumor effect in T47D cells via decreased phosphorylation of HER2, Akt, P13K and upregulation of p-p38 and p-JNK1/2. ${ }^{251}$ The compound has also been shown to induce caspase-3-dependent apoptosis in MDA-MB231 cells. $^{252} \alpha$-Mangostin inhibited fatty-acid synthase (FAS) expression and its intracellular accumulation, increased PARP cleavage, interfered with Bcl-2 family proteins and subsequently induced apoptosis in breast cancer cells. ${ }^{253}$ Won et al. (2014) demonstrated that growth inhibitory effect of $\alpha$-mangostin in $\mathrm{ER}+$ breast cancer cells is due to downregulation of estrogen receptor- $\alpha^{254}$ The compound encouraged mitochondrialmediated apoptosis and arrested cell cycle at G1 phase in p53 mutant and HER2, PgR, ER-negative breast cancer cells via downregulation of cylins, PCNA, CDKs and cdc(s) and increased p21 ${ }^{\mathrm{CIP} 1}$ expression. ${ }^{255}$

Parthenolide. A sesquiterpene lactone from feverfew herbcarries anti-inflammatory and anti-cancer properties. ${ }^{256-259}$ Parthenolide-mediated ROS generation in breast cancer cells led to the activation of both AMPK (autophagy-survival) and apoptotic pathways. Suppression of autophagy potentiated apoptosis by parthenolide. ${ }^{260}$ The compound enhanced production of ROS and superoxide anion via stimulation of EGFR in MDA-MB-231 cells. ${ }^{261}$ Parthenolide and dimethylaminoparthinolide (DMAPT i.e. a soluble analogue of parthenolide) strongly induced ROS generation in MDA-MB-231 (TNBC) cells 
and caused cell death. The drugs activated NADPH oxidase which led to the production of superoxide anion. ROS generation resulted in activation of JNK and suppression of NF- $\kappa \mathrm{B}$, as well as, depletion of glutathione and thiol groups. Additionally, the drugs induced autophagy by increased expression of Beclin1 and conversion of LC3-I to LC3-II. Finally, RIP-1 (signalling molecule in necrosis) expression was enhanced by the drugs. ${ }^{\mathbf{2 6}}$ Similarly, parthenolide and DMAPT exerted inhibitory effects on CSCs derived from TNBC cells via ROS generation, Nrf2 downregulation, mitochondrial dysfunction and CSCs necrosis. ${ }^{45}$ Suberoyanilide hydroxamic acid (SAHA) also sensitized TNBC (MDA-MB-231) cells to cytotoxicity of parthenolide via suppression of Akt/mTOR/Nrf2 pathway. Moreover, parthenolide inhibited the autophagic activity of SAHA and downregulation of $\mathrm{NF}-\kappa \mathrm{B}$ also ensued. ${ }^{263}$

3,3'-Diindolylmethane (DIM). A phytoestrogen and bioactive metabolite, derived from indole-3-carbinol-suppressed bisphenol A, triclosan (endocrine disrupting chemicals) and $17 \beta$-estradiol-mediated EMT, migration, invasion and proliferation of MCF-7 cells by regulating the expressions of metastasisand EMT-related genes. Furthermore, the phytoestrogen also reduced the expression of CXCR4-a receptor of CXCL12 chemokine that is responsible for breast cancer metastasis through ER-dependent pathway. ${ }^{264}$ DIM sensitized MDR MCF-7 cells to $\gamma$-radiation via increased G2/M phase arrest and intracellular ROS generation, along with enhanced apoptosis. ${ }^{265}$ The compound inhibited Akt activation, proliferation and migration in MDA-MB-231 cells via suppression of c-Met activation by hepatocyte growth factor. ${ }^{266}$ DIM increased the efficacy of Herceptin in HER-2/neu-expressing breast cancer cells by decreasing cell viability, associated with inhibition of colonization and apoptosis induction through downregulation of NF- $\kappa \mathrm{B}$ p65 and Akt. Mechanistic studies showed decreased FoxM1 expression and upregulation of miR-200. ${ }^{267}$

Luteolin. A citrus bioflavonoid found in various plants including parsley, thyme and peppermint-displayed antioxidant, anti-allergic, anti-tumor and anti-inflammatory activities. ${ }^{268-272}$ Metabolism of luteolin by cytochrome P450 enzymes CYP1A1 and CYP1B1 increased its anti-proliferative action in breast cancer cells. ${ }^{273}$ Luteolin inhibited migration of TNBC cells to the lungs and induced apoptosis via inhibition of VEGF. ${ }^{274}$ Moreover, the compound also reversed EMT via downregulation of $\beta$-catenin, thereby prevented lung metastasis of TNBC cells in xenograft model of breast cancer. ${ }^{275}$ Cotreatment of luteolin and lapatinib markedly suppressed the growth of BT-474 cells by reducing p-AKT and p-ERK1/2.276 Similarly, luteolin and celecoxib synergistically inhibited the growth of SkBr3 and MDA-MB-231 cells via inactivation of AKT and activation of ERK signalling, while in MCF-7 cells, inactivation of both ERK and Akt achieved the same result. ${ }^{277}$ Sensitization of drug-resistant MCF-7 cells to tamoxifen by luteolin via downregulation of cyclin E2 was also reported. ${ }^{278}$ However, low concentration of luteolin was shown to attenuate doxorubicin-induced cytotoxicity in MCF-7 cells through increased Bcl-2 expression. ${ }^{279}$

\section{Critical bottlenecks in clinical translation}

Although previous observations have directed various fruitful pre-clinical studies, still inadequate amount of clinical trials have been made to completely depict the distinct impact that each phytochemical may exert on cancer prevention. Several of these failures have been linked to the distribution of compounds, variable bioavailability and optimum mixtures of

Table 3 Bottlenecks in the clinical translation of phytochemicals

\begin{tabular}{ll}
\hline Phytochemical & Bottlenecks in clinical translation \\
\hline Curcumin & Poor bioavailability, quick metabolism, slow aqueous solubility \\
Resveratrol & Poor bioavailability, quick metabolism, lack of proper dose \\
EGCG & Poor bioavailability, low stability, metabolic transformation \\
& under physiological circumstances \\
Quercetin & Poor bioavailability, instability, low permeability, low solubility \\
Apigenin & Poor bioavailability, poor aqueous solubility, low lipid solubility \\
Kaempferol & Low bioavailability, poor solubility \\
Genistein & Poor water solubility, low serum level after oral delivery (bioavailability), \\
& bitter taste \\
Silibinin & Poor bioavailability, poor water and lipid solubility \\
Parthenolide & Poor bioavailability \& solubility in blood plasma, off-target effects, \\
& increased hydrophobicity \\
Sulforaphane & Instability, poor gastrointestinal absorption, hydrophobicity, \\
& poor bioavailability \\
$3,3^{\prime}$-Diindolylmethane & Poor absoption, poor biodistribution \\
Thymoquinone & Poor aqueous solubility, poor bioavailability, high lipophilicity \\
Naringenin & Poor bioavailability, instability, low aqueous solubility, low permeability, \\
& extensive first pass metabolism \\
Isoliquiritigenin & Poor bioavailability, poor solubility, low targeting ability, high effective dose \\
Ginsenosides & Poor oral absorption, poor bioavailability \\
Secoisolariciresinol & Poor bioavailability \\
Luteolin & Poor water solubility, poor bioavailability
\end{tabular}


phytochemicals. ${ }^{340}$ Bioavailability is the rate and degree to which an active moiety/ingredient is absorbed from a drug and becomes presented at the action site. ${ }^{341}$ When describing bioavailability, the mode of application of active ingredient also needs to be taken into consideration. In case of oral administration, both the digestive and metabolic processing must be taken into account while in case of intravenous application only the capacity to metabolize the nutrient by host organism must be considered. ${ }^{342-344}$ A major hurdle in phytochemical research is the absence of consensus about the required optimum dose of several of these agents to be employed in trials. Phytochemicals exhibit their optimal cancer preventive property at relevant doses. ${ }^{340}$

Several obstacles limit the clinical efficacy of curcumin such as poor bioavailabilty, quick metabolism and slow aqueous solubility. ${ }^{345,346}$ To improve the potential clinical efficacy of curcumin in high-risk populations, present research is mainly focusing on enhancing bioavailability to overcome both rapid compound metabolism and variability of absorption. Several efforts combining glucuronidation inhibitors (piperine, for instance) with curcumin to restrict intestinal and hepatic metabolism have revealed promising results. ${ }^{347}$ Other recent attempts have focused on using nanoparticles, curcumin analogs or delivery via phospholipid or liposomal complexing. ${ }^{348,349}$ A phase-I dose-escalation trial revealed that participants getting single dose of liposomal curcumin had a dosedependent enhancement in plasma level of curcumin, as well as, its active metabolite (tetrahydrocurcumin) without any clinical side effects. However, at higher doses of curcumin (120 $\mathrm{mg} \mathrm{m}^{-2}$ ), changes in morphology of RBC were seen, signifying a dose restricting sign of toxicity. ${ }^{350}$

Despite the promising anti-proliferative effects of resveratrol in vitro and in animal models, its translation to clinics also face several challenges. One obstacle is limited bioavailability as resveratrol is metabolically excluded from the body very fast, thereby creating difficulty in maintaining a therapeutically effective level in bloodstream. ${ }^{351}$ Recently, several efforts have been made in combining other natural substances with resveratrol to enhance its overall therapeutic value, especially cancer prevention. ${ }^{351}$ Pharmacokinetic studies of resveratrol showed extensive and rapid metabolism to resveratrol-3-Osulfate, resveratrol-3'-O-glucuronide and resveratrol-4'-O-glucuronide following oral administration at different doses, which doesn't allow for sufficient anticancer property of resveratrol. $^{352,353}$ Thus, efforts are underway to somehow sustain its metabolism in order to achieve better tissue exposure in the body. As resveratrol is rapidly metabolized into its metabolites, therefore, it is unclear whether metabolites may have different biological activity than free resveratrol or not. Resveratrol-3-Osulfate has also been found to be a chemopreventive agent. ${ }^{354}$ Another effort is to find the optimal route and dose of resveratrol. ${ }^{355}$

The main constituent of green tea i.e. EGCG, though extensively supported by results from cell culture, epidemiological, clinical and animal studies, however, there are several hurdles like bioavailability, stability and metabolic transformations under physiological circumstances. ${ }^{356}$ Several studies have displayed conflicting results concerning the cancer risk decreasing properties of green tea in different populations. This incompatibility in results may be because of variable tea preparations, variable bioavailability of compounds across populations and unknown concentration of antioxidants. ${ }^{340}$

Quercetin is also considered as a potent chemopreventive agent in various cancers, including breast cancer but its application in clinics is limited due to poor bioavailability, instability, low solubility and poor permeability. ${ }^{357}$ Several approaches have been developed to improve its bioavailability such as using nanoparticles, micelles, liposomes or inclusion complexes. Enhanced bioavailability will enable to bring this agent in forefront in disease therapeutics in the near future. ${ }^{357}$ Table 3 further depicts the bottlenecks involved in the clinical translation of various phytochemicals involved in breast cancer remedy.

\section{Conclusions and future perspective}

Breast cancer is challenging and account for maximum deaths across females in both developed and developing countries. As revealed by different epidemiological studies, there exists an inverse correlation between the use of phytochemicals and breast cancer incidence. Phytochemicals such as curcumin, resveratrol, silibinin, EGCG, thymoquinone, genistein, luteolin, $\alpha$-mangostin, luteolin, kaempferol, quercetin, parthenolide, apigenin, pterostilbene, isoliquiritigenin, DIM, ginsenosides and isothiocyanates possess great potential towards breast cancer chemoprevention. These phytochemicals display antibreast cancer activities through various mechanisms such as inhibition of proliferation, angiogenesis, migration, invasion, metastasis, as well as, induction of cell cycle arrest and apoptosis via modulation of several genes, gene products and signalling pathways. Furthermore, they have the potential to target breast cancer stem cells and overcome drugs resistance, along with sensitization to radiation and radio-protection. In recent years, much attention has been paid to natural remedies which have the capability to reduce adverse effects associated with currently available marketed drugs. Several synergistic approaches of phytochemicals with conventional chemotherapeutic drugs such as paclitaxel, doxorubicin, tamoxifen and adriamycin etc. have been addressed in various breast cancer cell lines for enhanced chemoprevention and sensitization.

High costs and adverse reactions associated with the use of conventional drugs and irradiations reduced their therapeutic efficacy. In this regard, phytochemicals provide a safer and costeffective approach. Most phytochemicals are readily available in vegetables, grains and fruits and their steady consumption would be effective to prevent breast cancer. It has been estimated that proper lifestyle adjustment could reduce cancer incidence by as much as two-thirds. Therefore, awareness should be raised among the general public about the proper and stable consumption of phytochemicals. Although, our understanding about multistage carcinogenesis has advanced, still the mechanism of action of most phytochemicals lacks adequate knowledge. The anticancer effects that most phytochemicals employ are probably the sum of several distinct 
mechanisms. Furthermore, deregulation or disruption of the intracellular signalling cascades sometime subject the cells to malignant transformation, so it is vital to recognize molecules in the signalling cascades that can be affected by specific phytochemicals in order to assess properly their underlying molecular mechanisms. Several phytochemicals have displayed non-toxic anti-tumor effects in xenograft model of nude mice, hence suggesting that they are effective in vivo.

A detailed insight about bioavailability and proper concentration of the required compounds are necessary, since most of them exerted dose-dependent inhibitory effects in breast cancer cells. Additionally, low concentration of some phytochemicals has shown to induce proliferation and activation of pro-survival autophagy in breast cancer, which further facilitate tumorigenesis. Likewise, large doses of phytochemicals resulted in development of additional diseases. For example, it has been shown that high doses of phytochemicals lead to chromosomal translocation, gastrointestinal disturbances and sexual dysfunction. ${ }^{377-379}$ Moreover, after absorption, phytochemicals are transformed to other conjugated forms, which may reduce their bioavailability. Thus, some alternations are required to enhance bioavailability of these phytochemicals, such as use of delivery systems like encapsulation, emulsion and other nanomedicine strategies. Pharmaceutical companies do not take interest in manufacturing phytochemical-based drugs due to intellectual property rights, which further creates hurdles. Some phytochemicals are produced by wild plants in relatively smaller proportions which make them difficult to be collected in large quantities, in which cases elicitation strategies and other plant tissue culture techniques provide a valuable insights for efficient accumulation of cancer related marker compounds, as well as, conservation of the respective plant species.

Strategies to decrease the side effects of chemotherapy are necessary, in which case, nanotechnology provides a useful opportunity in breast cancer therapy. The toxicity of conventional chemotherapeutic agents can be greatly reduced by administering them in nanoparticles and their combination with phytochemicals. Furthermore, new approach is required to enhance the intracellular stability, constant release and bioavailability of phytochemicals. ${ }^{380}$ Recently, it was reported that combination nanoparticles of paclitaxel and EGCG inhibited NF- $\mathrm{KB}$ activation, increased apoptosis, downregulated the genes involved in angiogenesis, cell survival and metastasis in MDA-MB-231 cell lines. Targeting the nanoparticles with anti-EGFR antibodies greatly enhanced these effects. Moreover, the EGCG-containing nanoparticles also overcame multidrug resistance in MDA-MB-231 cells via downregulation of P-glycoprotein. ${ }^{380}$ In another study, the intracellular concentration, stability and sustained release of EGCG was enhanced when loaded into chitosan-coated liposomes. The combined treatment significantly increased apoptosis and decreased cell viability. ${ }^{381}$ Similarly, quercetin encapsulation in MPEG-PLA (methoxypolyethylene glycol-polylactic acid) is useful in defeating its hydrophobicity. Quercetin nanoparticles induced apoptosis in MDA-MB-231 cells with sustained release for a duration of 10 days. $^{382}$ In another report, quercetin-loaded mesoporous silica nanoparticles with folic acid tag ensured increased bioavailability and targeted delivery in breast cancer cells. The nanostructure caused apoptosis and cell cycle arrest in breast cancer cells via regulation of Bax and Akt signalling. ${ }^{383}$ Curcumin exerts numerous anticancer effects, however, it is faced with several limitations such as low bioavailability, quick metabolism, quick degradation and low aqueous solubility. Nanotechnology is revealing promising results in improving the bioavailability of hydrophobic agents such as curcumin. It was observed that curcumin-loaded PLGA-PEG greatly improved the cytotoxic effects in MCF-7 cell lines as compared to pure curcumin. ${ }^{384}$ Recently, curcumin-loaded folate-modified chitosan nanoparticles having targeted ability were developed which displayed good potential for breast cancer therapy. It was observed that reducing the $\mathrm{pH}$ of the release medium increased the release rate of curcumin from nanoparticles, showing $\mathrm{pH}$ responsive capacity of modified nanoparticles. ${ }^{385}$ Curcuminloaded and calcium-doped dendritic mesoporous silica nanoparticles adapted with folic acid was also designed for effective treatment of breast cancer. This nanoformulation showed remarkable dispersal in aqueous solution, displayed $\mathrm{pH}$ responsive curcumin release and efficiently targeted the MCF-7 cells. The combination resulted in enhanced apoptosis, inhibition of proliferation, cell cycle arrest at G2/M phase, increased ROS generation and reduced mitochondrial membrane potential, in contrast to free curcumin. ${ }^{386}$ Resveratrol is also a promising candidate in breast cancer therapy, however, poor bioavailability and stability limit its clinical applications. Resveratrol-capped gold nanoparticles were synthesized previously, which repressed the TPA-induced invasion and migration abilities of breast cancer cells. The treatment greatly inhibited COX-2, ERK, MMP-9, NF-kB, P13K/Akt and AP-1. ${ }^{387}$ Resveratrol co-encapsulation with paclitaxel in a PEGylated liposome was successfully applied to drug resistant MCF-7/Adr cells which generated potent cytotoxicity. Moreover, the composite liposome also increased the bioavailability of the drug. ${ }^{388}$ In a recent study, resveratrol-load solid lipid nanoparticles were targeted on MDA-MB-231 cells which promoted Bax/Bcl-2 ratio and reduced the expression of c-Myc and cyclin D1, thereby greatly inhibiting proliferation, migration and invasion, as compared to free resveratrol. ${ }^{389}$ Thymoquinone-encapsulated nanoparticles were made by using hydrophilic and biodegradable polymers like PEG and PVP to overcome its poor aqueous solubility, minimal systemic bioavailability and light and thermal sensitivity. The resultant nanoparticles was more effective in killing cancer cells and less toxic to normal cells. Moreover, it displayed more potent anti-migratory activity on cancer cells. ${ }^{390}$ In another study, PLGA nanoparticles loaded with thymoquinone and paclitaxel were synthesized which showed improved anticancer activity in MCF-7 cells. This nanoformulation also aided in decreasing the toxic effects of paclitaxel by depressing its effective dosage. ${ }^{391}$ Similarly, nanostructured lipid carrier loading thymoquinone also improved the cytotoxicity and bioavailability of thymoquinone in MCF-7 and MDA-MB-231 cells. $^{392}$ These results demonstrate that nanoformulations based on phytochemicals provide a useful strategy to enhance their cytotoxicity, bioavailability, stability and sustained release, as well as, to overcome drugs resistance 
and other harmful side effects associated with conventional chemotherapeutic agents.

\section{Conflicts of interest}

All the authors declare no conflicts of interest, financial or otherwise, associated with this publication.

\section{References}

1 C. E. DeSantis, J. Ma, A. Goding Sauer, L. A. Newman and A. Jemal, Ca-Cancer J. Clin., 2017, 67, 439-448.

2 L. A. Torre, F. Bray, R. L. Siegel, J. Ferlay, J. Lortet-Tieulent and A. Jemal, Ca-Cancer J. Clin., 2015, 65, 87-108.

3 C. M. Perou and A.-L. Børresen-Dale, Cold Spring Harbor Perspect. Biol., 2011, 3, a003293.

4 J. S. Reis-Filho and L. Pusztai, Lancet, 2011, 378, 1812-1823. 5 T. Sørlie, C. M. Perou, R. Tibshirani, T. Aas, S. Geisler, H. Johnsen, T. Hastie, M. B. Eisen, M. Van De Rijn and S. S. Jeffrey, Proc. Natl. Acad. Sci. U. S. A., 2001, 98, 1086910874.

6 C. Sotiriou, S.-Y. Neo, L. M. McShane, E. L. Korn, P. M. Long, A. Jazaeri, P. Martiat, S. B. Fox, A. L. Harris and E. T. Liu, Proc. Natl. Acad. Sci. U. S. A., 2003, 100, 10393-10398.

7 C. A. Hudis and L. Gianni, Oncologist, 2011, 16, 1-11.

8 K. Reddy, Curr. Oncol., 2011, 18, e173.

9 S. J. Isakoff, Cancer J., 2010, 16, 53.

10 S. Moulder and G. Hortobagyi, Clin. Pharmacol. Ther., 2008, 83, 26-36.

11 M. Cazzaniga and B. Bonanni, BioMed Res. Int., 2012, 2012, 985620.

12 M. Wink, Medicines, 2015, 2, 251-286.

13 R. Verpoorte, A. Contin and J. Memelink, Phytochem. Rev., 2002, 1, 13-25.

14 H. N. Murthy, E.-J. Lee and K.-Y. Paek, Plant Cell, Tissue Organ Cult., 2014, 118, 1-16.

15 M. Wink, Theor. Appl. Genet., 1988, 75, 225-233.

16 M. Wink, T. Schmeller and B. Latz-Brüning, J. Chem. Ecol., 1998, 24, 1881-1937.

17 M. Wink and B.-E. Van Wyk, Mind-altering and poisonous plants of the world, Timber Press Portland, OR, 2008.

$18 \mathrm{M}$. Wink and O. Schimmer, Functions and biotechnology of plant secondary metabolites, 2010, vol. 39, pp. 21-161.

19 M. Wink, The Alkaloids: Chemistry and Biology, 2007, vol. 64, pp. 1-47.

20 M. Wink, in Studies in natural products chemistry, Elsevier, 2000, vol. 21, pp. 3-122.

21 R. H. Liu, Am. J. Clin. Nutr., 2003, 78, 517S-520S.

22 E. K. Arendt and E. Zannini, Cereal grains for the food and beverage industries, Elsevier, 2013.

23 Z.-h. Zhou, J. Yang and A.-N. Kong, Curr. Pharmacol. Rep., 2017, 3, 77-91.

24 M. Govindappa, J. Diabetes Metab., 2015, 6, 1000565.

25 B. Pagliaro, C. Santolamazza, F. Simonelli and S. Rubattu, BioMed Res. Int., 2015, 2015, 918069.
26 M. A. Islam, F. Alam, M. Solayman, M. I. Khalil, M. A. Kamal and S. H. Gan, Oxidative medicine and cellular longevity, 2016, vol. 2016.

27 A. Sunkaria, A. Yadav, S. K. Sharma and R. Sandhir, Neuroprotective Effects of Phytochemicals in Neurological Disorders, 2017, pp. 515-559.

28 A. M. Abbasi, M. Khan, M. Ahmad, M. Zafar, S. Jahan and S. Sultana, J. Ethnopharmacol., 2010, 128, 322-335.

29 M. S. Farvid, W. Y. Chen, K. B. Michels, E. Cho, W. C. Willett and A. H. Eliassen, BMJ, 2016, 353, i2343.

30 P. E. Miller and D. C. Snyder, Nutr. Clin. Pract., 2012, 27, 599-612.

31 M. K. Kim, J. H. Kim, S. J. Nam, S. Ryu and G. Kong, Nutr. Cancer, 2008, 60, 568-576.

32 C. D. Mock, B. C. Jordan and C. Selvam, RSC Adv., 2015, 5, $75575-75588$.

33 R. Venkatadri, C. Wright, V. Kaushik, Y. Kulkarni, J. S. Yakisich, A. K. V. Iyer and N. Azad, Cancer Res., 2015, 3801.

34 L. Le Corre, N. Chalabi, L. Delort, Y. J. Bignon and D. Bernard-Gallon, Mol. Nutr. Food Res., 2005, 49, 462-471.

35 E. C. Stuart, M. J. Scandlyn and R. J. Rosengren, Life Sci., 2006, 79, 2329-2336.

36 D. Sinha, J. Biswas, S. M. Nabavi and A. Bishayee, Semin. Cancer Biol., 2017, 46, 33-54.

37 M. Dastpeyman, N. Motamed, K. Azadmanesh, E. Mostafavi, V. Kia, A. Jahanian-Najafabadi and M. A. Shokrgozar, Med. Oncol., 2012, 29, 2512-2518.

38 D. Xiao, V. Vogel and S. V. Singh, Mol. Cancer Ther., 2006, 5, 2931-2945.

39 B. Xie, A. Nagalingam, P. Kuppusamy, N. Muniraj, P. Langford, B. Győrffy, N. K. Saxena and D. Sharma, Sci. Rep., 2017, 7, 40070.

40 D. F. Romagnolo, M. G. Donovan, A. J. Papoutsis, T. C. Doetschman and O. I. Selmin, Curr. Dev. Nutr., 2017, 1, e000562.

41 E. J. Choi and W. S. Ahn, Nutr. Res. Pract., 2008, 2, 322-325. 42 S. Li, T. Yan, R. Deng, X. Jiang, H. Xiong, Y. Wang, Q. Yu, X. Wang, C. Chen and Y. Zhu, OncoTargets Ther., 2017, 10, 4809.

43 M. Barkat, H. Abul, J. Ahmad, M. Khan, S. Beg and F. Ahmad, Curr. Drug Targets, 2018, 19, 70-80.

44 A. S. Sultan, M. I. Khalil, B. M. Sami, A. F. Alkhuriji and O. Sadek, Int. J. Clin. Exp. Pathol., 2017, 10, 156-172.

45 D. Carlisi, G. Buttitta, R. Di Fiore, C. Scerri, R. DragoFerrante, R. Vento and G. Tesoriere, Cell Death Dis., 2016, 7, e2194.

46 A. Pledgie-Tracy, M. D. Sobolewski and N. E. Davidson, Mol. Cancer Ther., 2007, 6, 1013-1021.

47 A. W. Harmon and Y. M. Patel, Breast Cancer Res. Treat., 2004, 85, 103-110.

48 K.-L. Wang, S.-M. Hsia, C.-J. Chan, F.-Y. Chang, C.-Y. Huang, D.-T. Bau and P. S. Wang, Expert Opin. Ther. Targets, 2013, 17, 337-349.

49 M. Maggiolini, G. Statti, A. Vivacqua, S. Gabriele, V. Rago, M. Loizzo, F. Menichini and S. Amdò, J. Steroid Biochem. Mol. Biol., 2002, 82, 315-322. 
50 Y. Cui, X.-O. Shu, Y.-T. Gao, H. Cai, M.-H. Tao and W. Zheng, Am. J. Epidemiol., 2006, 163, 645-653.

51 H. R. Shin, J. Y. Kim, T. K. Yun, G. Morgan and H. Vainio, Cancer Causes Control, 2000, 11, 565-576.

52 H. Lee, S. Lee, D. Jeong and S. J. Kim, J. Ginseng Res., 2017, DOI: 10.1016/j.jgr.2017.05.003.

53 M. Labbozzetta, M. Notarbartolo, P. Poma, A. Maurici, L. Inguglia, P. Marchetti, M. Rizzi, R. Baruchello, D. Simoni and N. D'Alessandro, Ann. N. Y. Acad. Sci., 2009, 1155, 278-283.

54 T. H. Kim, Y. J. Shin, A. J. Won, B. M. Lee, W. S. Choi, J. H. Jung, H. Y. Chung and H. S. Kim, Biochim. Biophys. Acta, Gen. Subj., 2014, 1840, 615-625.

55 N. S. Alamolhodaei, A. M. Tsatsakis, M. Ramezani, A. W. Hayes and G. Karimi, Food Chem. Toxicol., 2017, 103, 223-232.

56 T. Nabekura, Toxins, 2010, 2, 1207-1224.

57 J. F. Weiss and M. R. Landauer, Toxicology, 2003, 189, 1-20.

58 F. H. Sarkar and Y. Li, Cancer Res., 2006, 66, 3347-3350.

59 B. T. Kawasaki, E. M. Hurt, T. Mistree and W. L. Farrar, Mol. Interventions, 2008, 8, 174.

60 P. R. Dandawate, D. Subramaniam, R. A. Jensen and S. Anant, Semin. Cancer Biol., 2016, 40, 192-208.

61 S.-Y. Yi, Y.-B. Hao, K.-J. Nan and T.-L. Fan, Cancer Treat. Rev., 2013, 39, 290-296.

62 R. Pardal, M. F. Clarke and S. J. Morrison, Nat. Rev. Cancer, 2003, 3, 895-902.

63 R. C. Petric, C. Braicu, L. Raduly, O. Zanoaga, N. Dragos, P. Monroig, D. Dumitrascu and I. Berindan-Neagoe, OncoTargets Ther., 2015, 8, 2053.

64 J. Akhtar Siddiqui, A. Singh, M. Chagtoo, N. Singh, M. Madhav Godbole and B. Chakravarti, Curr. Cancer Drug Targets, 2015, 15, 116-135.

65 B. J. Cridge, L. Larsen and R. J. Rosengren, Oncology Discovery, 2013, 1, 6.

66 X. Wang, Y. Hang, J. Liu, Y. Hou, N. Wang and M. Wang, Oncol. Lett., 2017, 13, 4825-4831.

67 B. L. Regassa and A. Vaidya, Breast Cancer Manage., 2016, 5, 93-106.

68 Z.-D. Lv, X.-P. Liu, W.-J. Zhao, Q. Dong, F.-N. Li, H.-B. Wang and B. Kong, Int. J. Clin. Exp. Pathol., 2014, 7, 2818.

69 H. Fan, Y. Liang, B. Jiang, X. Li, H. Xun, J. Sun, W. He, H. T. Lau and X. Ma, Oncol. Rep., 2016, 35, 2651-2656.

70 B. Chen, Y. Zhang, Y. Wang, J. Rao, X. Jiang and Z. Xu, J. Steroid Biochem. Mol. Biol., 2014, 143, 11-18.

71 S. Mukherjee, M. Mazumdar, S. Chakraborty, A. Manna, S. Saha, P. Khan, P. Bhattacharjee, D. Guha, A. Adhikary and S. Mukhjerjee, Stem Cell Res. Ther., 2014, 5, 116.

72 U. Kumar, U. Sharma and G. Rathi, Tumor Biol., 2017, 39, DOI: $10.1177 / 1010428317692258$.

73 Y. Liu, J. Zhou, Y. Hu, J. Wang and C. Yuan, Mol. Cell. Biochem., 2017, 425, 47-58.

74 L. Du, Z. Xie, L.-c. Wu, M. Chiu, J. Lin, K. K. Chan, S. Liu and Z. Liu, Nutr. Cancer, 2012, 64, 1228-1235.

75 R. R. Falah, W. H. Talib and S. J. Shbailat, Ther. Adv. Med. Oncol., 2017, 9, 235-252.
76 H. Moghtaderi, H. Sepehri and F. Attari, Biomed. Pharmacother., 2017, 88, 582-594.

77 P. Odonmažig, A. Ebringerová, E. Machová and J. Alföldi, Carbohydr. Res., 1994, 252, 317-324.

78 M. Ren, Y. Wang, X. Wu, S. Ge and B. Wang, J. Drug Targeting, 2017, 25, 247-254.

79 P. Thulasiraman, G. Garriga, V. Danthuluri, D. J. McAndrews and I. Q. Mohiuddin, Oncol. Rep., 2017, 37, 2007-2015.

80 L. Xiao-ai, W. Bei, X. Xiao-hong, P. Lei, W. Bin, D. Xiao-xue, Z. Chen-hui and D. Qi-wei, RSC Adv., 2017, 7, 33572-33579.

81 B. Vinod, J. Antony, H. Nair, V. Puliyappadamba, M. Saikia, S. S. Narayanan, A. Bevin and R. J. Anto, Cell Death Dis., 2013, 4, e505.

82 H. Zong, F. Wang, Q.-x. Fan and L.-x. Wang, Mol. Biol. Rep., 2012, 39, 4803-4808.

83 K. Sun, X. Duan, H. Cai, X. Liu, Y. Yang, M. Li, X. Zhang and J. Wang, Clin. Exp. Med., 2016, 16, 37-47.

84 S. Bimonte, A. Barbieri, G. Palma, D. Rea, A. Luciano, M. D'Aiuto, C. Arra and F. Izzo, BioMed Res. Int., 2015, 2015, 878134.

85 S.-F. Hendrayani, H. H. Al-Khalaf and A. Aboussekhra, Neoplasia, 2013, 15, 631IN611-640IN611.

86 Z. K. Hassan and M. H. Daghestani, Asian Pac. J. Cancer Prev., 2012, 13, 3259-3264.

87 N. Mo, Z.-Q. Li, J. Li and Y.-D. Cao, Asian Pac. J. Cancer Prev., 2012, 13, 5709-5714.

88 M. Gallardo and G. M. Calaf, Int. J. Oncol., 2016, 49, 10191027.

89 W. Zhu, L. Jia, G. Chen, H. Zhao, X. Sun, X. Meng, X. Zhao, L. Xing, J. Yu and M. Zheng, Oncotarget, 2016, 7, 48607.

90 M. Bonucci, M. Fioranelli, M. G. Roccia and T. Lotti, Dermatol. Ther., 2017, 30, e12493.

91 H. Zhao, W. Zhu, L. Jia, X. Sun, G. Chen, X. Zhao, X. Li, X. Meng, L. Kong and L. Xing, Br. J. Radiol., 2015, 89, 20150665.

92 M. Moradzadeh, A. Hosseini, S. Erfanian and H. Rezaei, Pharmacol. Rep., 2017, 69, 924-928.

93 C.-Y. Huang, Z. Han, X. Li, H.-H. Xie and S.-S. Zhu, Oncol. Lett., 2017, 14, 3623-3627.

94 O. Y. Hong, E. M. Noh, H. Y. Jang, Y. R. Lee, B. K. Lee, S. H. Jung, J. S. Kim and H. J. Youn, Oncol. Lett., 2017, 14, 441-446.

95 R. León-Cachón, J. Ascacio-Martínez and H. A. BarreraSaldaña, Rev. Invest. Clin., 2012, 64, 364-376.

96 F. De Amicis, A. Russo, P. Avena, M. Santoro, A. Vivacqua, D. Bonofiglio, L. Mauro, S. Aquila, D. Tramontano and S. A. Fuqua, Mol. Nutr. Food Res., 2013, 57, 840-853.

97 L. Zeng, J. M. Holly and C. M. Perks, Front. Endocrinol., 2014, 5, 61.

98 X. Pan, B. Zhao, Z. Song, S. Han and M. Wang, J. Pharmacol. Sci., 2016, 130, 85-93.

99 S. Sun, Y. Dai, Z. Lu, M. Li, Z. Zhai, X. Ren and D. Li, Int. J. Clin. Exp. Pathol., 2016, 9, 4251-4259.

100 M. A. Esmaeili, Journal of Chemical Biology, 2016, 9, 41-52.

101 A. Nowakowska and J. Tarasiuk, Acta Biochim. Pol., 2016, 63, 571-575. 
102 D. G. Pons, M. Nadal-Serrano, M. Torrens-Mas, J. Oliver and P. Roca, J. Cell. Biochem., 2016, 117, 218-229.

103 E. J. Choi, J. Y. Jung and G.-H. Kim, Exp. Ther. Med., 2014, 8, 454-458.

104 Y. Li, S. M. Meeran, S. N. Patel, H. Chen, T. M. Hardy and T. O. Tollefsbol, Mol. Cancer, 2013, 12, 9.

105 E. J. Choi and G.-H. Kim, Mol. Med. Rep., 2013, 7, 781-784. 106 Q. Zhao, M. Zhao, A. B. Parris, Y. Xing and X. Yang, Int. J. Oncol., 2016, 49, 1203-1210.

107 Y. Fang, Q. Zhang, X. Wang, X. Yang, X. Wang, Z. Huang, Y. Jiao and J. Wang, Int. J. Oncol., 2016, 48, 1016-1028.

108 Q. Xie, Q. Bai, L. Y. Zou, Q. Y. Zhang, Y. Zhou, H. Chang, L. Yi, J. D. Zhu and M. T. Mi, Genes, Chromosomes Cancer, 2014, 53, 422-431.

109 R. R. Jadhav, J. Santucci-Pereira, Y. V. Wang, J. Liu, T. D. Nguyen, J. Wang, S. Jenkins, J. Russo, T. H.-M. Huang and V. X. Jin, Genes, 2017, 8, 144.

110 X. Zhang, K. L. Cook, A. Warri, I. M. Cruz, M. Rosim, J. Riskin, W. Helferich, D. Doerge, R. Clarke and L. Hilakivi-Clarke, Clin. Cancer Res., 2017, 23, 814-824.

111 C. B. Avci, S. Y. Susluer, H. O. Caglar, T. Balci, D. Aygunes, Y. Dodurga and C. Gunduz, Contemp. Oncol., 2015, 19, 32.

112 J. Sims-Mourtada, L. Opdenaker, J. Davis and C. Wu, Cancer Stud. Mol. Med., 2015, 2, 60-65.

113 P. Fan, S. Fan, H. Wang, J. Mao, Y. Shi, M. M. Ibrahim, W. Ma, X. Yu, Z. Hou and B. Wang, Stem Cell Res. Ther., 2013, 4, 146.

114 E. Izquierdo-Torres, G. Rodríguez, I. Meneses-Morales and A. Zarain-Herzberg, Mol. Carcinog., 2017, 56, 1703-1711.

115 A. Khan, A. N. Aljarbou, Y. H. Aldebasi, S. M. Faisal and M. A. Khan, Cancer Epidemiol., 2014, 38, 765-772.

116 Y. N. Kim, S. R. Choe, K. H. Cho, D. Y. Cho, J. Kang, C. G. Park and H. Y. Lee, Exp. Mol. Med., 2017, 49, e296.

117 R. Medina-Aguilar, L. A. Marchat, E. Arechaga Ocampo, P. Gariglio, J. García Mena, N. Villegas Sepúlveda, M. Martínez Castillo and C. López-Camarillo, Oncol. Rep., 2016, 35, 3696-3704.

118 O. H. Alobaedi, W. H. Talib and I. A. Basheti, Asian Pac. J. Trop. Med., 2017, 10, 400-408.

119 R. Venkatadri, A. K. V. Iyer, V. Kaushik and N. Azad, Pharmacol. Rep., 2017, 69, 788-797.

120 R. Kala and T. O. Tollefsbol, PLoS One, 2016, 11, e0155057. 121 A. Mondal and L. L. Bennett, Biomed. Pharmacother., 2016, 84, 1906-1914.

122 A. Alayev, S. M. Berger, M. Y. Kramer, N. S. Schwartz and M. K. Holz, J. Cell. Biochem., 2015, 116, 450-457.

123 S. Chottanapund, M. Van Duursen, P. Navasumrit, P. Hunsonti, S. Timtavorn, M. Ruchirawat and M. Van den Berg, Toxicol. in Vitro, 2014, 28, 1215-1221.

124 F. Huang, X.-N. Wu, J. Chen, W.-X. Wang and Z. F. Lu, Exp. Ther. Med., 2014, 7, 1611-1616.

125 J. Díaz-Chávez, M. A. Fonseca-Sánchez, E. ArechagaOcampo, A. Flores-Pérez, Y. Palacios-Rodríguez, G. Domínguez-Gómez, L. A. Marchat, L. Fuentes-Mera, G. Mendoza-Hernández and P. Gariglio, PLoS One, 2013, 8, e64378.
126 X.-P. Shi, S. Miao, Y. Wu, W. Zhang, X.-F. Zhang, H.-Z. Ma, H.-L. Xin, J. Feng, A.-D. Wen and Y. Li, Int. J. Mol. Sci., 2013, 14, 15655-15668.

127 F. Casanova, J. Quarti, D. C. F. da Costa, C. A. Ramos, J. L. da Silva and E. Fialho, J. Cell. Biochem., 2012, 113, 2586-2596.

128 R. Venkatadri, T. Muni, A. Iyer, J. Yakisich and N. Azad, Cell Death Dis., 2016, 7, e2104.

129 D. Pradhan, R. K. Pradhan, G. Tripathy and S. Pradhan, J. Young Pharm., 2015, 7, 225.

130 L. Khorsandi, M. Orazizadeh, F. Niazvand, M. Abbaspour, E. Mansouri and A. Khodadadi, Bratisl. Lek. Listy, 2017, 118, 123-128.

131 S. Ranganathan, D. Halagowder and N. D. Sivasithambaram, PLoS One, 2015, 10, e0141370.

132 X.-H. Deng, H.-Y. Song, Y.-F. Zhou, G.-Y. Yuan and F.-J. Zheng, Exp. Ther. Med., 2013, 6, 1155-1158.

133 F. Ramezani, N. Samadi and Z. Mostafavi-Pour, Nutr. Cancer, 2017, 69, 881-891.

134 H. Wang, L. Tao, K. Qi, H. Zhang, D. Feng, W. Wei, H. Kong, T. Chen and Q. Lin, J. BUON, 2015, 20, 707-713.

135 C. Huang, S.-Y. Lee, C.-L. Lin, T.-H. Tu, L. H. Chen, Y. J. Chen and H.-C. Huang, J. Agric. Food Chem., 2013, 61, 6430-6445.

136 A. Srinivasan, C. Thangavel, Y. Liu, S. Shoyele, R. B. Den, P. Selvakumar and A. Lakshmikuttyamma, Mol. Carcinog., 2016, 55, 743-756.

137 X. Zhao, Q. Wang, S. Yang, C. Chen, X. Li, J. Liu, Z. Zou and D. Cai, Eur. J. Pharmacol., 2016, 781, 60-68.

138 S.-f. Tao, H.-f. He and Q. Chen, Mol. Cell. Biochem., 2015, 402, 93-100.

139 M. Younas, S. Drouet, M. Nadeem, N. Giglioli-Guivarc'h, C. Hano and B. H. Abbasi, J. Photochem. Photobiol., B, 2018, 184, 61-70.

140 Y. Wang, W.-C. Liang, W.-L. Pan, W.-K. Law, J.-S. Hu, D. T.-M. Ip, M. M.-Y. Waye, T.-B. Ng and D. C.-C. Wan, Phytomedicine, 2014, 21, 1310-1317.

141 S.-J. Oh, S. P. Jung, J. Han, S. Kim, J. S. Kim, S. J. Nam, J. E. Lee and J.-H. Kim, Oncol. Rep., 2013, 29, 1343-1348.

142 K. Alimoghaddam and A. Ghavamzadeh, Arch. Iran. Med., 2014, 17, 366.

143 M. B. Pirouzpanah, M. Sabzichi, S. Pirouzpanah, H. Chavoshi and N. Samadi, Asian Pac. J. Cancer Prev., 2015, 16, 2087-2092.

144 W. H. Kil, S. M. Kim, J. E. Lee, K. S. Park and S. J. Nam, Ann. Surg. Treat. Res., 2014, 87, 167-173.

145 H. Chavoshi, V. Vahedian, S. Saghaei, M. B. Pirouzpanah, M. Raeisi and N. Samadi, Asian Pac. J. Cancer Prev., 2017, 18, 2243.

146 O. Molavi, F. Narimani, F. Asiaee, S. Sharifi, V. Tarhriz, A. Shayanfar, M. Hejazi and R. Lai, Pharm. Biol., 2017, 55, 729-739.

147 Z. J. Maasomi, Y. P. Soltanahmadi, M. Dadashpour, S. Alipour, S. Abolhasani and N. Zarghami, Asian Pac. J. Cancer Prev., 2017, 18, 1283.

148 M. M. Zadeh, N. Motamed, N. Ranji, M. Majidi and F. Falahi, J. Breast Cancer, 2016, 19, 45-52. 
149 Z. Jahanafrooz, N. Motamed and B. Bakhshandeh, Cytotechnology, 2017, 1-14.

150 K. Jiang, W. Wang, X. Jin, Z. Wang, Z. Ji and G. Meng, Oncol. Rep., 2015, 33, 2711-2718.

151 N. Zheng, P. Zhang, H. Huang, W. Liu, T. Hayashi, L. Zang, Y. Zhang, L. Liu, M. Xia and S.-i. Tashiro, J. Pharmacol. Sci., 2015, 128, 97-107.

152 M. Marjaneh, M. Narazah and H. Shahrul, Apoptosis, 2015, 1, 10001014.

153 B. Saraçligil, B. Öztürk, Ș. B. Bozkurt and Y. Kahveci, Int. J. Pharm. Sci. Res., 2017, 8, 2849-2852.

154 S. Rajput, B. Kumar, P. Banik, S. Parida and M. Mandal, J. Cell. Physiol., 2015, 230, 620-629.

155 S. Parbin, A. Shilpi, S. Kar, N. Pradhan, D. Sengupta, M. Deb, S. K. Rath and S. K. Patra, Mol. BioSyst., 2016, 12, 48-58.

156 S. Ganji-Harsini, M. Khazaei, Z. Rashidi and A. Ghanbari, Cell, 2016, 18, 245.

157 Ç. Şakalar, K. İzgi, B. İskender, S. Sezen, H. Aksu, M. Çakır, B. Kurt, A. Turan and H. Canatan, Tumor Biol., 2016, 37, 4467-4477.

158 L. H. Odeh, W. H. Talib and I. A. Basheti, J. Cancer Res. Ther., 2018, 14, 324.

159 A. Sehrawat, C. S. Croix, C. J. Baty, S. Watkins, D. Tailor, R. P. Singh and S. V. Singh, Mitochondrion, 2016, 30, 67-77.

160 E. J. Kim, S. J. Eom, J. E. Hong, J.-Y. Lee, M.-S. Choi and J. H. Y. Park, Mol. Cell. Biochem., 2012, 359, 431-440.

161 S.-H. Kim, A. Sehrawat and S. V. Singh, Cancer Prev. Res., 2013, 6, 782-790.

162 S.-H. Kim and S. V. Singh, Breast Cancer Res. Treat., 2015, 149, 681-692.

163 D. Bauer, N. Redmon, E. Mazzio and K. F. Soliman, PLoS One, 2017, 12, e0175558.

164 G. Soria, M. Ofri-Shahak, I. Haas, N. Yaal-Hahoshen, L. Leider-Trejo, T. Leibovich-Rivkin, P. Weitzenfeld, T. Meshel, E. Shabtai and M. Gutman, BMC Cancer, 2011, 11, 130.

165 A. Papi, G. Storci, T. Guarnieri, S. De Carolis, S. Bertoni, N. Avenia, A. Sanguinetti, A. Sidoni, D. Santini and C. Ceccarelli, PLoS One, 2013, 8, e54968.

166 A. Ben-Baruch, Cancer Microenviron., 2012, 5, 151-164.

167 J. L. Steiner and E. A. Murphy, Int. J. Biol. Markers, 2012, 27, e179-185.

168 B. Kindlund, Å. Sjöling, C. Yakkala, J. Adamsson, A. Janzon, L.-E. Hansson, M. Hermansson, P. Janson, O. Winqvist and S. B. Lundin, Gastric Cancer, 2017, 20, 116-125.

169 S. Tabariès, V. Ouellet, B. E. Hsu, M. G. Annis, A. A. Rose, L. Meunier, E. Carmona, C. E. Tam, A.-M. Mes-Masson and P. M. Siegel, Breast Cancer Res., 2015, 17, 45.

170 P. Mishra, D. Banerjee and A. Ben-Baruch, J. Leukocyte Biol., 2011, 89, 31-39.

171 S. A. Ibrahim, G. K. Katara, A. Kulshrestha, M. K. Jaiswal, M. A. Amin and K. D. Beaman, Oncotarget, 2015, 6, 33033.

172 C. N. Vrakas, R. M. O'Sullivan, S. E. Evans, D. A. Ingram, C. B. Jones, T. Phuong and R. A. Kurt, Immunol. Invest., 2015, 44, 174-188.
173 H. S. Seo, J. M. Ku, H. S. Choi, J. K. Woo, B. H. Lee, D. S. Kim, H. J. Song, B. H. Jang, Y. C. Shin and S. G. Ko, Oncol. Rep., 2017, 38, 715-724.

174 H.-S. Seo, J. K. Jo, J. M. Ku, H.-S. Choi, Y. K. Choi, J.-K. Woo, S.-y. Kang, K. min Lee, K. W. Nam and N. Park, Biosci. Rep., 2015, 35, e00276.

175 H.-S. Seo, H.-S. Choi, S.-R. Kim, Y. K. Choi, S.-M. Woo, I. Shin, J.-K. Woo, S.-Y. Park, Y. C. Shin and S.-K. Ko, Mol. Cell. Biochem., 2012, 366, 319-334.

176 T. H. Tseng, M. H. Chien, W. L. Lin, Y. C. Wen, J. M. Chow, C. K. Chen, T. C. Kuo and W. J. Lee, Environ. Toxicol., 2017, 32, 434-444.

177 M. E. Harrison, M. R. P. Coombs, L. M. Delaney and D. W. Hoskin, Exp. Mol. Pathol., 2014, 97, 211-217.

178 G. Chen, L. Zhu, Y. Liu, Q. Zhou, H. Chen and J. Yang, Phytother. Res., 2009, 23, 498-506.

179 G. Chen, X. Hu, W. Zhang, N. Xu, F.-Q. Wang, J. Jia, W.-F. Zhang, Z.-J. Sun and Y.-F. Zhao, Apoptosis, 2012, 17, 90-101.

180 F. Peng, Q. Du, C. Peng, N. Wang, H. Tang, X. Xie, J. Shen and J. Chen, Phytother. Res., 2015, 29, 969-977.

181 F. Peng, H. Tang, P. Liu, J. Shen, X. Guan, X. Xie, J. Gao, L. Xiong, L. Jia and J. Chen, Sci. Rep., 2017, 7, 9022.

182 L. Si, X. Yang, X. Yan, Y. Wang and Q. Zheng, Oncol. Lett., 2017, 14, 241-249.

183 S. K. Lee, K.-K. Park, K. R. Kim, H.-J. Kim and W.-Y. Chung, Journal of Cancer Prevention, 2015, 20, 281.

184 S. Ning, X. Ma, D. Zhu, Z. Shen, J. Liu, Y. Liu, J. Chen and Z. Li, RSC Adv., 2017, 7, 18085-18092.

185 S. Ning, J. Mu, Z. Shen, D. Zhu, F. Jiang, X. Wang, Y. Li and Z. Li, RSC Adv., 2016, 6, 24719-24727.

186 Z. Wang, N. Wang, P. Liu, Q. Chen, H. Situ, T. Xie, J. Zhang, C. Peng, Y. Lin and J. Chen, Oncotarget, 2014, 5, 7013.

187 N. Wang, Z. Wang, Y. Wang, X. Xie, J. Shen, C. Peng, J. You, F. Peng, H. Tang and X. Guan, Oncotarget, 2015, 6, 9854.

188 N. Wang, Z. Wang, C. Peng, J. You, J. Shen, S. Han and J. Chen, Carcinogenesis, 2014, 35, 2544-2554.

189 R. Wakimoto, M. Ono, M. Takeshima, T. Higuchi and S. Nakano, Anticancer Res., 2017, 37, 6153-6159.

190 C.-M. Hung, L.-C. Liu, C.-T. Ho, Y.-C. Lin and T.-D. Way, J. Agric. Food Chem., 2017, 65, 11179-11191.

191 C.-M. Su, W.-H. Lee, A. T. Wu, Y.-K. Lin, L.-S. Wang, C.-H. Wu and C.-T. Yeh, J. Nutr. Biochem., 2015, 26, 675685.

192 M. Guarino, J. Cell. Physiol., 2010, 223, 14-26.

193 A. Varkaris, A. D. Katsiampoura, J. C. Araujo, G. E. Gallick and P. G. Corn, Cancer Metastasis Rev., 2014, 33, 595-606.

194 L. C. Kim, L. Song and E. B. Haura, Nat. Rev. Clin. Oncol., 2009, 6, 587-595.

195 C.-H. Wu, B.-H. Hong, C.-T. Ho and G.-C. Yen, J. Agric. Food Chem., 2015, 63, 2432-2441.

196 K. K. Mak, A. T. Wu, W. H. Lee, T. C. Chang, J. F. Chiou, L. S. Wang, C. H. Wu, C. Y. F. Huang, Y. S. Shieh and T. Y. Chao, Mol. Nutr. Food Res., 2013, 57, 1123-1134.

197 L. Kong, B. Liu, C. Zhang, B. Wang, H. Wang, X. Song, Y. Yang, X. Ren, L. Yin and H. Kong, Neurochem. Int., 2016, 100, 52-61. 
198 M. Lenzi, C. Fimognari and P. Hrelia, in Advances in Nutrition and Cancer, Springer, 2014, pp. 207-223.

199 S. M. Tortorella, S. G. Royce, P. V. Licciardi and T. C. Karagiannis, Antioxid. Redox Signaling, 2015, 22, 1382-1424.

200 J. D. Clarke, R. H. Dashwood and E. Ho, Cancer Lett., 2008, 269, 291-304.

201 E. Heiss, C. Herhaus, K. Klimo, H. Bartsch and C. Gerhäuser, J. Biol. Chem., 2001, 276, 32008-32015.

202 Y. W. An, K. A. Jhang, S.-Y. Woo, J. L. Kang and Y. H. Chong, Neurobiol. Aging, 2016, 38, 1-10.

203 H. Tilg, Clin. Phytosci., 2015, 1, 10.

204 J. P. Burnett, G. Lim, Y. Li, R. B. Shah, R. Lim, H. J. Paholak, S. P. McDermott, L. Sun, Y. Tsume and S. Bai, Cancer Lett., 2017, 394, 52-64.

205 S. H. Kim, H. J. Park and D. O. Moon, Oncol. Lett., 2017, 13, 4427-4432.

206 C. Bao, J. Ko, H.-C. Park, M. C. Kim, J. Kim, J.-H. Auh and H. J. Lee, Food Sci. Biotechnol., 2015, 24, 347-351.

207 Y.-R. Lee, E.-M. Noh, J.-H. Han, J.-M. Kim, B.-M. Hwang, B.-S. Kim, S.-H. Lee, S. H. Jung, E. Y. Chung and J.-S. Kim, BMB Rep., 2013, 46, 201.

208 L. L. Atwell, L. M. Beaver, J. Shannon, D. E. Williams, R. H. Dashwood and E. Ho, Curr. Pharmacol. Rep., 2015, 1, 102-111.

209 K. J. Royston, N. Udayakumar, K. Lewis and T. O. Tollefsbol, Int. J. Mol. Sci., 2017, 18, 1092.

210 A. Lewinska, J. Adamczyk-Grochala, A. Deregowska and M. Wnuk, Theranostics, 2017, 7, 3461.

211 H. Yu, C. Zhang, M. Lu, F. Sun, Y. Fu and F. Jin, Chem. Pharm. Bull., 2007, 55, 231-235.

212 J.-M. Lu, Q. Yao and C. Chen, Curr. Vasc. Pharmacol., 2009, 7, 293-302.

213 J. Y. Park, H. You, D. Lee, W. Huh, G. S. Hwang, K. T. No, K. H. Kim, J. Ham, N. Yamabe and Y. Kim, Bull. Korean Chem. Soc., 2016, 37, 52-55.

214 X. Wen, H.-D. Zhang, L. Zhao, Y.-F. Yao, J.-H. Zhao and J.-H. Tang, Asian Pac. J. Cancer Prev., 2015, 16, 1105-1109.

215 Z. Yuan, H. Jiang, X. Zhu, X. Liu and J. Li, Biomed. Pharmacother., 2017, 89, 227-232.

216 B.-M. Kim, D.-H. Kim, J.-H. Park, Y.-J. Surh and H.-K. Na, Journal of Cancer Prevention, 2014, 19, 23.

217 Y. Zhang, Q.-Z. Liu, S.-P. Xing and J.-L. Zhang, Asian Pac. J. Trop. Med., 2016, 9, 180-183.

218 Y. Zou and P. Liu, Int. J. Clin. Exp. Med., 2016, 9, 1766417669.

219 S.-J. Kim and A. K. Kim, J. Ginseng Res., 2015, 39, 125-134.

220 Y. Di, F. De Silva, E. S. Krol and J. Alcorn, Nutr. Cancer, 2018, 1-10.

221 Y. Zhu, K. Kawaguchi and R. Kiyama, PLoS One, 2017, 12, e0171390.

222 L. Schröder, D. U. Richter, B. Piechulla, M. Chrobak, C. Kuhn, S. Schulze, S. Abarzua, U. Jeschke and T. Weissenbacher, Nutrients, 2016, 8, 616.

223 D. Ilbeigi, M. Nourbakhsh, S. Khaghani, N. Einollahi, N. Kheiripour, Z. Gholinejad, M. Alaee and M. Saberian, Cell, 2017, 19, 37.
224 X.-Y. Xiong, X.-J. Hu, Y. Li and C.-M. Liu, Nutr. Cancer, 2015, 67, 1326-1334.

225 A. V. Mali, A. A. Joshi, M. V. Hegde and S. S. Kadam, Asian Pac. J. Cancer Prev., 2017, 18, 905.

226 D. M. Delman, C. J. Fabian, B. F. Kimler, H. Yeh and B. K. Petroff, Nutr. Cancer, 2015, 67, 857-864.

227 P. Seibold, A. Vrieling, T. S. Johnson, K. Buck, S. Behrens, R. Kaaks, J. Linseisen, N. Obi, J. Heinz and D. Flesch-Janys, Int. J. Cancer, 2014, 135, 923-933.

228 C. Kyrø, L. Hansen, K. Frederiksen, N. P. Nørskov, K. E. B. Knudsen, A. K. Eriksen, M. Holm, A. Tjønneland and A. Olsen, Clin. Nutr., 2017, DOI: 10.1016/ j.clnu.2017.10.023.

229 S. Jaskulski, A. Y. Jung, A. Rudolph, T. Johnson, K. Thöne, E. Herpel, P. Sinn and J. Chang-Claude, Mol. Nutr. Food Res., 2017, 61, 1700449.

230 S. E. McCann, S. B. Edge, D. G. Hicks, L. U. Thompson, C. D. Morrison, G. Fetterly, C. Andrews, K. Clark, J. Wilton and S. Kulkarni, Nutr. Cancer, 2014, 66, 566-575.

231 G. Flower, H. Fritz, L. G. Balneaves, S. Verma, B. Skidmore, R. Fernandes, D. Kennedy, K. Cooley, R. Wong and S. Sagar, Integr. Cancer Ther., 2014, 13, 181-192.

232 S. Dutta, P. S. Kharkar, N. U. Sahu and A. Khanna, Life Sci., 2017, 185, 73-84.

233 S. Esmaeili-Mahani, F. Falahi and M. M. Yaghoobi, J. Evidence-Based Complementary Altern. Med., 2014, 2014, 893247.

234 S. Esmaeili-Mahani, M. R. Samandari-Bahraseman and M. M. Yaghoobi, Iran. J. Pharm. Res., 2018, 17, 343.

235 T. Ghanbari, M. Azadbakht, A. Vesi-Raygani and M. Khazaei, Int. J. Morphol., 2016, 34, 1197-1202.

236 F. Zeng, R.-J. Ju, L. Liu, H.-J. Xie, L.-M. Mu, Y. Zhao, Y. Yan, Y.-J. Hu, J.-S. Wu and W.-L. Lu, Oncotarget, 2015, 6, 36625. 237 C.-T. Lee, Y.-W. Huang, C.-H. Yang and K.-S. Huang, Curr. Top. Med. Chem., 2015, 15, 1491-1500.

238 R. C. Wang, X. Chen, A. M. Parissenti, A. A. Joy, J. Tuszynski, D. N. Brindley and Z. Wang, PLoS One, 2017, 12, e0182400.

239 C. Luchsinger, M. Aguilar, P. V. Burgos, P. Ehrenfeld and G. A. Mardones, PLoS One, 2018, 13, e0195401.

240 P. Ghanbari, M. Mohseni, M. Tabasinezhad, B. Yousefi, A. A. Saei, S. Sharifi, M. R. Rashidi and N. Samadi, Appl. Biochem. Biotechnol., 2014, 174, 667-681.

241 C. Azevedo, A. Correia-Branco, J. R. Araújo, J. T. Guimarães, E. Keating and F. Martel, Nutr. Cancer, 2015, 67, 504-513.

242 X. Yi, J. Zuo, C. Tan, S. Xian, C. Luo, S. Chen, L. Yu and Y. Luo, Afr. J. Tradit., Complementary Altern. Med., 2016, 13, 210-215.

243 S.-H. Kim, K.-A. Hwang and K.-C. Choi, J. Nutr. Biochem., 2016, 28, 70-82.

244 G.-A. Lee, K.-C. Choi and K.-A. Hwang, Environ. Toxicol. Pharmacol., 2017, 49, 48-57.

245 K. Matsumoto, Y. Akao, H. Yi, K. Ohguchi, T. Ito, T. Tanaka, E. Kobayashi, M. Iinuma and Y. Nozawa, Bioorg. Med. Chem., 2004, 12, 5799-5806.

246 K. Matsumoto, Y. Akao, K. Ohguchi, T. Ito, T. Tanaka, M. Iinuma and Y. Nozawa, Bioorg. Med. Chem., 2005, 13, 6064-6069. 
247 Y. Akao, Y. Nakagawa and Y. Nozawa, Int. J. Mol. Sci., 2008, 9, 355-370.

248 P. Moongkarndi, N. Kosem, S. Kaslungka, O. Luanratana, N. Pongpan and N. Neungton, J. Ethnopharmacol., 2004, 90, 161-166.

249 C.-H. Lee, T.-H. Ying, H.-L. Chiou, S.-C. Hsieh, S.-H. Wen, R.-H. Chou and Y.-H. Hsieh, Oncotarget, 2017, 8, 47425.

250 M.-A. Shibata, M. Iinuma, J. Morimoto, H. Kurose, K. Akamatsu, Y. Okuno, Y. Akao and Y. Otsuki, BMC Med., 2011, 9, 69.

251 S. Kritsanawong, S. Innajak, M. Imoto and R. Watanapokasin, Int. J. Oncol., 2016, 48, 2155-2165.

252 N. Atluri, V. Thirumalanadhuni and U. M. devi Palempalli, Int. J. Phytomed., 2014, 6, 354-359.

253 P. Li, W. Tian and X. Ma, Mol. Cancer, 2014, 13, 138.

254 Y.-S. Won, J.-H. Lee, S.-J. Kwon, J.-Y. Kim, K.-H. Park, M.-K. Lee and K.-I. Seo, Food Chem. Toxicol., 2014, 66, 158-165.

255 H. Kurose, M.-A. Shibata, M. Iinuma and Y. Otsuki, BioMed Res. Int., 2012, 2012, 672428.

256 S. P. Hehner, T. G. Hofmann, W. Dröge and M. L. Schmitz, J. Immunol., 1999, 163, 5617-5623.

257 V. B. Mathema, Y.-S. Koh, B. C. Thakuri and M. Sillanpää, Inflammation, 2012, 35, 560-565.

258 A. Ghantous, A. Sinjab, Z. Herceg and N. Darwiche, Drug discovery today, 2013, 18, 894-905.

259 A. Pareek, M. Suthar, G. S. Rathore and V. Bansal, Pharmacogn. Rev., 2011, 5, 103.

260 C. Lu, W. Wang, Y. Jia, X. Liu, Z. Tong and B. Li, J. Cell. Biochem., 2014, 115, 1458-1466.

261 A. D'Anneo, D. Carlisi, S. Emanuele, G. Buttitta, R. Di Fiore, R. Vento, G. Tesoriere and M. Lauricella, Int. J. Oncol., 2013, 43, 1895-1900.

262 A. D'anneo, D. Carlisi, M. Lauricella, R. Puleio, R. Martinez, S. Di Bella, P. Di Marco, S. Emanuele, R. Di Fiore and A. Guercio, Cell Death Dis., 2013, 4, e891.

263 D. Carlisi, M. Lauricella, A. D'Anneo, G. Buttitta, S. Emanuele, R. Di Fiore, R. Martinez, C. Rolfo, R. Vento and G. Tesoriere, J. Cell. Physiol., 2015, 230, 1276-1289.

264 G.-A. Lee, K.-A. Hwang and K.-C. Choi, Food Chem. Toxicol., 2017, 109, 284-295.

265 W. Wang, M. Lv, Y. Wang and J. Zhang, Pharm. Biol., 2016, 54, 3164-3168.

266 H. L. Nicastro, G. L. Firestone and L. F. Bjeldanes, J. Nutr. Biochem., 2013, 24, 1882-1888.

267 A. Ahmad, S. Ali, A. Ahmed, A. S. Ali, A. Raz, W. A. Sakr and K. W. Rahman, PLoS One, 2013, 8, e54657.

268 E. R. Kasala, L. N. Bodduluru, C. C. Barua and R. Gogoi, Biomed. Pharmacother., 2016, 82, 568-577.

269 J. Xiong, K. Wang, C. Yuan, R. Xing, J. Ni, G. Hu, F. Chen and X. Wang, Int. J. Mol. Med., 2017, 39, 113-125.

270 G. Seelinger, I. Merfort and C. M. Schempp, Planta Med., 2008, 74, 1667-1677.

271 L. Ziyan, Z. Yongmei, Z. Nan, T. Ning and L. Baolin, Planta Med., 2007, 73, 221-226.

272 M. Kimata, N. Inagaki and H. Nagai, Planta Med., 2000, 66, 25-29.
273 N. E. Wilsher, R. R. Arroo, M. T. Matsoukas, A. M. Tsatsakis, D. A. Spandidos and V. P. Androutsopoulos, Food Chem. Toxicol., 2017, 110, 383-394.

274 M. T. Cook, Y. Liang, C. Besch-Williford and S. M. Hyder, Breast Cancer: Targets Ther., 2017, 9, 9.

275 D. Lin, G. Kuang, J. Wan, X. Zhang, H. Li, X. Gong and H. Li, Oncol. Rep., 2017, 37, 895-902.

276 L. Zhang, F. Yang, L. Huang, A. Liu and J. Zhang, Biomed. Res., 2017, 28, 4902-4907.

277 Y. W. Jeon, Y. E. Ahn, W. S. Chung, H. J. Choi and Y. J. Suh, Tumor Biol., 2015, 36, 6349-6359.

278 S.-H. Tu, C.-T. Ho, M.-F. Liu, C.-S. Huang, H.-W. Chang, C.-H. Chang, C.-H. Wu and Y.-S. Ho, Food Chem., 2013, 141, 1553-1561.

279 Y. Sato, N. Sasaki, M. Saito, N. Endo, F. Kugawa and A. Ueno, Biol. Pharm. Bull., 2015, 38, 703-709.

280 F. Guan, Y. Ding, Y. Zhang, Y. Zhou, M. Li and C. Wang, PLoS One, 2016, 11, e0146553.

281 C. Geng, J. Li, F. Ding, G. Wu, Q. Yang, Y. Sun, Z. Zhang, T. Dong and X. Tian, Biochem. Biophys. Res. Commun., 2016, 473, 147-153.

282 J. A. Colacino, S. P. McDermott, M. A. Sartor, M. S. Wicha and L. S. Rozek, Breast Cancer Res. Treat., 2016, 158, 29-41.

283 Ö. Berrak, Y. Akkoç, E. D. Arısan, A. Çoker-Gürkan, P. Obakan-Yerlikaya and N. Palavan-Ünsal, Biomed. Pharmacother., 2016, 77, 150-160.

284 E. Kronski, M. E. Fiori, O. Barbieri, S. Astigiano, V. Mirisola, P. H. Killian, A. Bruno, A. Pagani, F. Rovera and U. Pfeffer, Mol. Oncol., 2014, 8, 581-595.

285 T. Huang, Z. Chen and L. Fang, Oncol. Rep., 2013, 29, 117124.

286 M. Jiang, O. Huang, X. Zhang, Z. Xie, A. Shen, H. Liu, M. Geng and K. Shen, Molecules, 2013, 18, 701-720.

287 H.-W. Lai, S.-Y. Chien, S.-J. Kuo, L.-M. Tseng, H.-Y. Lin, C.-W. Chi and D.-R. Chen, J. Evidence-Based Complementary Altern. Med., 2012, 2012, 486568.

288 X.-D. Sun, X.-E. Liu and D.-S. Huang, Mol. Med. Rep., 2012, 6, 1267-1270.

289 J.-M. Kim, E.-M. Noh, K.-B. Kwon, J.-S. Kim, Y.-O. You, J.-K. Hwang, B.-M. Hwang, B.-S. Kim, S.-H. Lee and S. J. Lee, Phytomedicine, 2012, 19, 1085-1092.

290 M. Lazzeroni, A. Guerrieri-Gonzaga, S. Gandini, H. Johansson, D. Serrano, M. Cazzaniga, V. Aristarco, D. Macis, S. Mora and P. Caldarella, Cancer Prev. Res., 2017, 10, 363-370.

291 K. Hallman, K. Aleck, M. Quigley, B. Dwyer, V. Lloyd, M. Szmyd and S. Dinda, Breast Cancer: Targets Ther., $2017,9,365$.

292 G. Deb, V. S. Thakur, A. M. Limaye and S. Gupta, Mol. Carcinog., 2015, 54, 485-499.

293 N. D. Mineva, K. E. Paulson, S. P. Naber, A. S. Yee and G. E. Sonenshein, PLoS One, 2013, 8, e73464.

294 C. Braicu, C. D. Gherman, A. Irimie and I. BerindanNeagoe, J. Nanosci. Nanotechnol., 2013, 13, 632-637.

295 H. Luo, M. Xu, W. Zhong, Z. Cui, F. Liu, K. Zhou and X. Li, J. BUON, 2014, 19, 435-439. 
296 J.-Y. Jang, J.-K. Lee, Y.-K. Jeon and C.-W. Kim, BMC Cancer, 2013, 13, 421.

297 S. Mirza, G. Sharma, R. Parshad, S. D. Gupta, P. Pandya and R. Ralhan, J. Breast Cancer, 2013, 16, 23-31.

298 J.-P. Xue, G. Wang, Z.-B. Zhao, Q. Wang and Y. Shi, Oncol. Rep., 2014, 32, 1647-1653.

299 X. Liu, C. Sun, X. Jin, P. Li, F. Ye, T. Zhao, L. Gong and Q. Li, Molecules, 2013, 18, 13200-13217.

300 T. T. Rajah, K. J. Peine, N. Du, C. A. Serret and N. R. Drews, Anticancer Res., 2012, 32, 1181-1191.

301 H. Pan, W. Zhou, W. He, X. Liu, Q. Ding, L. Ling, X. Zha and S. Wang, Int. J. Mol. Med., 2012, 30, 337-343.

302 J. Saluzzo, K. M. Hallman, K. Aleck, B. Dwyer, M. Quigley, V. Mladenovik, A. E. Siebert and S. Dinda, Genes Cancer, 2016, 7, 414.

303 Y. Fu, H. Chang, X. Peng, Q. Bai, L. Yi, Y. Zhou, J. Zhu and M. Mi, PLoS One, 2014, 9, e102535.

304 L. S. Gomez, P. Zancan, M. C. Marcondes, L. Ramos-Santos, J. R. Meyer-Fernandes, M. Sola-Penna and D. Da Silva, Biochimie, 2013, 95, 1336-1343.

305 S.-H. Tu, K.-C. Yang, C.-T. Ho, C.-S. Huang, C.-S. Chen, H.-W. Chang, C.-H. Chang, C.-H. Wu and Y.-S. Ho, Journal of Cancer Research and Practice, 2013, 29, 167-181.

306 J. Duo, G.-G. Ying, G.-W. Wang and L. Zhang, Mol. Med. Rep., 2012, 5, 1453-1456.

307 G. Lee, K.-A. Hwang and K.-C. Choi, Environ. Toxicol. Pharmacol., 2017, 49, 48-57.

308 K. M. Perrott, C. D. Wiley, P.-Y. Desprez and J. Campisi, GeroScience, 2017, 39, 161-173.

309 X. Cao, B. Liu, W. Cao, W. Zhang, F. Zhang, H. Zhao, R. Meng, L. Zhang, R. Niu and X. Hao, Chin. J. Cancer Res., 2013, 25, 212.

310 M. Karimi, H. Babaahmadi-Rezaei, G. Mohammadzadeh and M.-A. Ghaffari, Iran. J. Pathol., 2017, 12, 135-143.

311 Z. Jahanafrooz, N. Motameh and B. Bakhshandeh, Asian Pac. J. Cancer Prev., 2016, 17, 2661-2665.

312 T. H. Kim, J. S. Woo, Y. K. Kim and K. H. Kim, J. Pharmacol. Exp. Ther., 2014, 349, 268-278.

313 W. Lu, C. Lin, T. D. King, H. Chen, R. C. Reynolds and Y. Li, Cell. Signalling, 2012, 24, 2291-2296.

314 B. H. Hong, C. H. Wu, C. T. Yeh and G. C. Yen, Mol. Nutr. Food Res., 2013, 57, 886-895.

315 A. Hussain, M. Javeria, A. P. Sathyen, B. Salema, N. Qurrat El-Ain, H. Geetganga, J. Elham, M. Ali Khan and C. Sharma, Asian Pac. J. Cancer Prev., 2013, 14, 5855-5860. 316 J. Sui, K. Xie and M. Xie, Shengli Xuebao, 2016, 68, 27-34.

317 M. T. Cook, Y. Liang, C. Besch-Williford, S. Goyette, B. Mafuvadze and S. M. Hyder, SpringerPlus, 2015, 4, 444.

318 D.-W. Sun, H.-D. Zhang, L. Mao, C.-F. Mao, W. Chen, M. Cui, R. Ma, H.-X. Cao, C.-W. Jing and Z. Wang, Cell. Physiol. Biochem., 2015, 37, 1693-1711.

319 E.-J. Lee, S.-Y. Oh and M.-K. Sung, Food Chem. Toxicol., 2012, 50, 4136-4143.

320 M. J. Kim, J. S. Woo, C. H. Kwon, J. H. Kim, Y. K. Kim and K. H. Kim, Cell Biol. Int., 2012, 36, 339-344.

321 L.-M. Wang, K.-P. Xie, H.-N. Huo, F. Shang, W. Zou and M.-J. Xie, Asian Pac. J. Cancer Prev., 2012, 13, 1431-1437.
322 L. Eanes and Y. M. Patel, Biochimie Open, 2016, 3, 64-71.

323 F. Zhang, W. Dong, W. Zeng, L. Zhang, C. Zhang, Y. Qiu, L. Wang, X. Yin, C. Zhang and W. Liang, Breast Cancer Res., 2016, 18, 38.

324 Y. Sun and J. Gu, Zhongguo Zhongyao Zazhi, 2015, 40, 11441150.

325 M. S. I. Abaza, K. Y. Orabi, E. Al-Quattan and J. Raja'a, Cancer Cell Int., 2015, 15, 46.

326 J.-Y. Ke, Y.-H. Hsiao, S. R. Straka, L. D. Yee and M. A. Belury, Cancer Res., 2015, 903.

327 J. Helle, K. Kräker, M. I. Bader, A. M. Keiler, O. Zierau, G. Vollmer, J. Welsh and G. Kretzschmar, Mol. Cell. Endocrinol., 2014, 392, 125-135.

328 T. Hatkevich, J. Ramos, I. Santos-Sanchez and Y. M. Patel, Exp. Cell Res., 2014, 327, 331-339.

329 S. Kim and T. I. Park, Int. J. Clin. Exp. Med., 2013, 6, 890.

330 P. Bulzomi, A. Bolli, P. Galluzzo, F. Acconcia, P. Ascenzi and M. Marino, IUBMB Life, 2012, 64, 690-696.

331 M. N. Dastjerdi, E. M. Mehdiabady, F. G. Iranpour and H. Bahramian, Int. J. Prev. Med., 2016, 7, 66.

332 S. Rajput, B. P. Kumar, K. K. Dey, I. Pal, A. Parekh and M. Mandal, Life Sci., 2013, 93, 783-790.

333 H. Zheng, Y. Li, Y. Wang, H. Zhao, J. Zhang, H. Chai, T. Tang, J. Yue, A. M. Guo and J. Yang, Toxicol. Appl. Pharmacol., 2014, 280, 10-20.

334 M. Marques, L. Laflamme, I. Benassou, C. Cissokho, B. Guillemette and L. Gaudreau, BMC Cancer, 2014, 14, 524.

335 P. Wang, X. Du, M. Xiong, J. Cui, Q. Yang, W. Wang, Y. Chen and T. Zhang, Sci. Rep., 2016, 6, 33709.

336 S.-H. Kim, Y. Wan and S. V. Singh, Cancer Res., 2017, 5265. 337 S.-H. Kim, A. Sehrawat and S. V. Singh, Breast Cancer Res. Treat., 2012, 134, 1067-1079.

338 D. Xiao, A. Bommareddy, S.-H. Kim, A. Sehrawat, E.-R. Hahm and S. V. Singh, PLoS One, 2012, 7, e32597.

339 H. Li, B. Yang, J. Huang, T. Xiang, X. Yin, J. Wan, F. Luo, L. Zhang, H. Li and G. Ren, Toxicol. Lett., 2013, 220, 219228.

340 R. Kotecha, A. Takami and J. L. Espinoza, Oncotarget, 2016, $7,52517$.

341 S. W. Hoag and A. S. Hussain, J. Nutr., 2001, 131, 1389S1391S.

342 M.-M. Mocanu, P. Nagy and J. Szöllősi, Molecules, 2015, 20, 22578-22620.

343 V. S. Srinivasan, J. Nutr., 2001, 131, 1349S-1350S.

344 R. P. Heaney, J. Nutr., 2001, 131, 1344S-1348S.

345 P. Anand, A. B. Kunnumakkara, R. A. Newman and B. B. Aggarwal, Mol. Pharmaceutics, 2007, 4, 807-818.

346 A. Shehzad, F. Wahid and Y. S. Lee, Arch. Pharm., 2010, 343, 489-499.

347 G. Shoba, D. Joy, T. Joseph, M. M. R. Rajendran and P. Srinivas, Planta Med., 1998, 64, 353-356.

348 Q. Huang, H. Yu and Q. Ru, J. Food Sci., 2010, 75, R50-R57. 349 S. Prasad, A. K. Tyagi and B. B. Aggarwal, Cancer Res. Treat., 2014, 46, 2.

350 A. Storka, B. Vcelar, U. Klickovic, G. Gouya, S. Weisshaar, S. Aschauer, G. Bolger, L. Helson and M. Woltz, Int. J. Clin. Pharmacol. Ther., 2015, 53, 54-65. 
351 C. K. Singh, M. A. Ndiaye and N. Ahmad, Biochim. Biophys. Acta, Mol. Basis Dis., 2015, 1852, 1178-1185.

352 D. J. Boocock, G. E. Faust, K. R. Patel, A. M. Schinas, V. A. Brown, M. P. Ducharme, T. D. Booth, J. A. Crowell, M. Perloff and A. J. Gescher, Cancer Epidemiol. Biomark. Prev., 2007, 16, 1246-1252.

353 L. Almeida, M. Vaz-da-Silva, A. Falcão, E. Soares, R. Costa, A. I. Loureiro, C. Fernandes-Lopes, J. F. Rocha, T. Nunes and L. Wright, Mol. Nutr. Food Res., 2009, 53, S7-S15.

354 J. Hoshino, E.-J. Park, T. P. Kondratyuk, L. Marler, J. M. Pezzuto, R. B. Van Breemen, S. Mo, Y. Li and M. Cushman, J. Med. Chem., 2010, 53, 5033-5043.

355 P. R. Van Ginkel, D. Sareen, L. Subramanian, Q. Walker, S. R. Darjatmoko, M. J. Lindstrom, A. Kulkarni, D. M. Albert and A. S. Polans, Clin. Cancer Res., 2007, 13, 5162-5169.

356 C. Huo, S. Wan, W. Lam, L. Li, Z. Wang, K. Landis-Piwowar, D. Chen, Q. Dou and T. Chan, Inflammopharmacology, 2008, 16, 248-252.

357 X. Cai, Z. Fang, J. Dou, A. Yu and G. Zhai, Curr. Med. Chem., 2013, 20, 2572-2582.

358 J. Zhang, D. Liu, Y. Huang, Y. Gao and S. Qian, Int. J. Pharm., 2012, 436, 311-317.

359 S.-m. Ding, Z.-h. Zhang, J. Song, X.-d. Cheng, J. Jiang and X.-b. Jia, Int. J. Nanomed., 2014, 9, 2327.

360 K. Zhang, L. Gu, J. Chen, Y. Zhang, Y. Jiang, L. Zhao, K. Bi and X. Chen, J. Pharm. Biomed. Anal., 2015, 114, 168-175.

361 H.-Y. Si, D.-P. Li, T.-M. Wang, H.-L. Zhang, F.-Y. Ren, Z.-G. Xu and Y.-Y. Zhao, J. Nanosci. Nanotechnol., 2010, 10, 2325-2331.

362 R. Cohen, B. Schwartz, I. Peri and E. Shimoni, J. Agric. Food Chem., 2011, 59, 7932-7938.

363 N. Dixit, S. Baboota, K. Kohli, S. Ahmad and J. Ali, Indian J. Pharmacol., 2007, 39, 172.

364 Y. Song, J. Zhuang, J. Guo, Y. Xiao and Q. Ping, Pharmazie, 2008, 63, 35-42.

365 C. A. Houghton, R. G. Fassett and J. S. Coombes, Nutr. Rev., 2013, 71, 709-726.

366 H. Danafar, A. Sharafi, H. Kheiri Manjili and S. Andalib, Pharm. Dev. Technol., 2017, 22, 642-651.

367 M. Paltsev, V. Kiselev, E. Muyzhnek, V. Drukh, I. Kuznetsov and O. Pchelintseva, EPMA J., 2013, 4, 25.

368 M. Elmowafy, A. Samy, M. A. Raslan, A. Salama, R. A. Said, A. E. Abdelaziz, W. El-Eraky, S. El Awdan and T. Viitala, AAPS PharmSciTech, 2016, 17, 663-672.

369 F. Odeh, S. I. Ismail, R. Abu-Dahab, I. S. Mahmoud and A. Al Bawab, Drug Delivery, 2012, 19, 371-377.

370 F.-L. Yen, T.-H. Wu, L.-T. Lin, T.-M. Cham and C.-C. Lin, Pharm. Res., 2009, 26, 893-902.

371 D. V. Ratnam, D. Ankola, V. Bhardwaj, D. K. Sahana and M. R. Kumar, J. Controlled Release, 2006, 113, 189-207.

372 M. Cuendet, J. Guo, Y. Luo, S. Chen, C. P. Oteham, R. C. Moon, R. B. Van Breemen, L. E. Marler and J. M. Pezzuto, Cancer Prev. Res., 2010, 1940-6207.

373 F. Gao, J. Zhang, C. Fu, X. Xie, F. Peng, J. You, H. Tang, Z. Wang, P. Li and J. Chen, Int. J. Nanomed., 2017, 12, 4147.
374 Z. Yang, S. Gao, J. Wang, T. Yin, Y. Teng, B. Wu, M. You, Z. Jiang and M. Hu, Drug Metab. Dispos., 2011, 111, 040006.

375 A. Gupta, L. D. Kagliwal and R. S. Singhal, in Advances in food and nutrition research, Elsevier, 2013, vol. 69, pp. 183-217.

376 H. Dang, M. H. W. Meng, H. Zhao, J. Iqbal, R. Dai, Y. Deng and F. Lv, J. Nanopart. Res., 2014, 16, 2347.

377 G. Galati and P. J. O'brien, Free Radicals Biol. Med., 2004, 37, 287-303.

378 R. Strick, P. L. Strissel, S. Borgers, S. L. Smith and J. D. Rowley, Proc. Natl. Acad. Sci. U. S. A., 2000, 97, 47904795.

379 H. L. Bonkovsky, Ann. Intern. Med., 2006, 144, 68.

380 S. Narayanan, U. Mony, D. K. Vijaykumar, M. Koyakutty, B. Paul-Prasanth and D. Menon, Nanomedicine, 2015, 11, 1399-1406.

381 R. C. C. de Pace, X. Liu, M. Sun, S. Nie, J. Zhang, Q. Cai, W. Gao, X. Pan, Z. Fan and S. Wang, J. Liposome Res., 2013, 23, 187-196.

382 G. Sharma, J. Park, A. R. Sharma, J.-S. Jung, H. Kim, C. Chakraborty, D.-K. Song, S.-S. Lee and J.-S. Nam, Pharm. Res., 2015, 32, 723-735.

383 A. Sarkar, S. Ghosh, S. Chowdhury, B. Pandey and P. C. Sil, Biochim. Biophys. Acta, Gen. Subj., 2016, 1860, 2065-2075.

384 F. S. Tabatabaei Mirakabad, A. Akbarzadeh, M. Milani, N. Zarghami, M. Taheri-Anganeh, V. Zeighamian, F. Badrzadeh and M. Rahmati-Yamchi, Artif. Cells, Nanomed., Biotechnol., 2016, 44, 423-430.

385 S. Esfandiarpour-Boroujeni, S. Bagheri-Khoulenjani, H. Mirzadeh and S. Amanpour, Carbohydr. Polym., 2017, 168, 14-21.

386 J. Wang, Y. Wang, Q. Liu, L. Yang, R. Zhu, C. Yu and S. Wang, ACS Appl. Mater. Interfaces, 2016, 8, 26511-26523.

387 S. Y. Park, S. Y. Chae, J. O. Park, K. J. Lee and G. Park, Oncol. Rep., 2016, 35, 3248-3256.

388 J. Meng, F. Guo, H. Xu, W. Liang, C. Wang and X.-D. Yang, Sci. Rep., 2016, 6, 22390.

389 W. Wang, L. Zhang, T. Chen, W. Guo, X. Bao, D. Wang, B. Ren, H. Wang, Y. Li and Y. Wang, Molecules, 2017, 22, 1814.

390 S. Bhattacharya, M. Ahir, P. Patra, S. Mukherjee, S. Ghosh, M. Mazumdar, S. Chattopadhyay, T. Das, D. Chattopadhyay and A. Adhikary, Biomaterials, 2015, 51, 91-107.

391 P. Soni, J. Kaur and K. Tikoo, J. Nanopart. Res., 2015, 17, 18. 392 W. K. Ng, L. Saiful Yazan, L. H. Yap, W. N. Hafiza, W. A. Ghani, C. W. How and R. Abdullah, BioMed Res. Int., 2015, 2015, 263131.

393 Y. Bellik, L. Boukraâ, H. A. Alzahrani, B. A. Bakhotmah, F. Abdellah, S. M. Hammoudi and M. Iguer-Ouada, Molecules, 2012, 18, 322-353.

394 A. A. Ramos, C. F. Lima and C. Pereira-Wilson, in Selected Topics in DNA Repair, InTech, 2011.

395 A. Abdal Dayem, H. Y. Choi, G.-M. Yang, K. Kim, S. K. Saha and S.-G. Cho, Nutrients, 2016, 8, 581.

396 D. Liu and Z. Chen, J. Breast Cancer, 2013, 16, 133-137.

397 L. Varinska, P. Gal, G. Mojzisova, L. Mirossay and J. Mojzis, Int. J. Mol. Sci., 2015, 16, 11728-11749. 
398 D. Sinha, N. Sarkar, J. Biswas and A. Bishayee, Semin. Cancer Biol., 2016, 40, 209-232.

399 R. H. Himes, Pharmacol. Ther., 1991, 51, 257-267.
400 M. Moudi, R. Go, C. Y. S. Yien and M. Nazre, Int. J. Prev. Med., 2013, 4, 1231.

401 C. Vassallo, A. Carugno, F. Derlino, O. Ciocca, V. Brazzelli and G. Borroni, Cutis, 2015, 95, E28-E34. 